\title{
Laboratory Directed Research and Development Program FY 2010
}

May 2011 


\section{DISCLAIMER}

This document was prepared as an account of work sponsored by the United States Government. While this document is believed to contain correct information, neither the United States Government nor any agency thereof, nor The Regents of the University of California, nor any of their employees, makes any warranty, express or implied, or assumes any legal responsibility for the accuracy, completeness, or usefulness of any information, apparatus, product, or process disclosed, or represents that its use would not infringe privately owned rights. Reference herein to any specific commercial product, process, or service by its trade name, trademark, manufacturer, or otherwise, does not necessarily constitute or imply its endorsement, recommendation, or favoring by the United States Government or any agency thereof, or The Regents of the University of California. The views and opinions of authors expressed herein do not necessarily state or reflect those of the United States Government or any agency thereof or The Regents of the University of California.

Lawrence Berkeley Laboratory is an equal opportunity employer. 


\author{
Report on \\ Ernest Orlando Lawrence \\ Berkeley National Laboratory
}

\title{
Laboratory Directed Research and Development Program
}

\section{FY 2010}

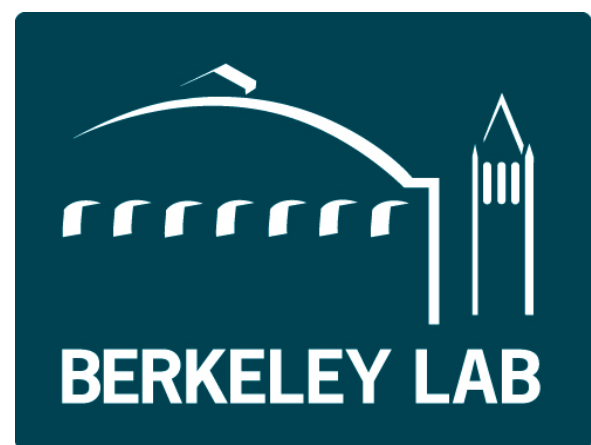

Ernest Orlando Lawrence Berkeley National Laboratory Berkeley, CA 94720

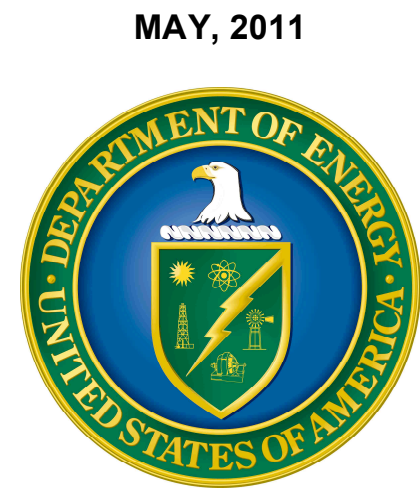

Prepared for the U.S. Department of Energy under Contract No. DE-AC02-05CH11231 



\section{Table of Contents}

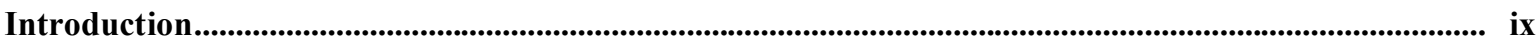

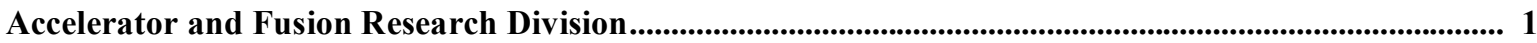

André Anders $\quad$ Plasma-Assisted High Rate Deposition Concept for Energy 1

Applications

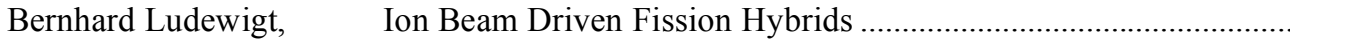

Peter Seidl

David Robin Novel Accelerator and Engineering Strategies for Ion Beam

Cancer Therapy ...................................................................... 3

Fernando Sannibale Experimental Accelerator R\&D Towards a Future Source at LBNL ......................................................................... 4

Thomas Schenkel Quantum Information Science with Integrated Color Centers in Diamond .......................................................................... 5

Csaba Toth Experimental Demonstration of a Laser-Plasma-Accelerator

Carl Schroeder

Driven Free-Electron Laser

2

William Fawley

Kem Robinson

Jean-Luc Vay, William Fawley

Lorentz Compaction of Scales for Ultra-Efficient Simulation of

Advanced Accelerators (and Other Systems)

Marco Venturini

Linac Driver and Coherent Soft X-ray Sources ......

Advanced Light Source Division

Elke Arenholz, Soren Prestemon

Alexander Hexemer et al

Alexander Hexemer,

Howard Padmore,

Ting $\mathrm{Xu}$,

Thomas Russell

David Kilcoyne

Martin Kunz, Nobumichi Tamura

Stefano Marchesini et al

Howard Padmore

Eli Rotenberg, Aaron Bostwick
Optimization of Flux Pinning in Type II Superconductor Based Magnets for Soft X-ray Scattering Applications .......................... 9

Soft X-ray Scattering as a New Probe of Polymer Systems .......... 10

Long-Range Ordering of Block Copolymers on Faceted Silicon... 11

Search for a Permanent Electron Electric Dipole Moment (EDM).....

Structure Solution of Inorganic Materials Using Energy

Resolved Laue Microdiffraction...

Nanoscale Surveyor ...

High Quantum Yield Multi-Alkali Cathodes for psec Pulsed Electron Sources

An Improved Electron Detection System for "Extreme" Angle-

Resolved Photoemission Experiments. 16 
Hendrik Bluhm,

Zahid Hussain,

Tolek Tyliszczak

Tony Warwick

Test Monochromator/ Spectrometer Systems with Prototype

High Density Gratings for High Resolution X-ray Scattering

Kenneth Goldberg,

X-Ray Optical Metrology for Coherence-Preserving Adaptive

Valeriy Yashcuk

Optics.

Chemical Sciences Division

Dmitry Budker,

Direct Comb Spectroscopy of Lithium in the Vacuum

Holger Mueller

Ultraviolet and Beyond.

Oliver Gessner

Probing Transient Molecular Entanglement Using Femtosecond

High Resolution Delayed-field Coincidence Imaging ....

Mary Gilles

Development of In Situ Cells for Reactive Spectroscopic and Microscopic Studies

Charles Harris

Dynamics of Homogeneous Catalysis Reactions Investigated with Transient Two-Dimensional Infrared Spectroscopy

Wayne Lukens

Quantifying f-electron Exchange Coupling in Actinide and Lanthanide Complexes

Thorsten Weber

Double-Auger Emission of Small Molecules Following Core-

Excitation and Ionization

\section{Computing Sciences}

(National Energy Research Scientific Computing Center, and Computational Research Divisions) .. 26

Deb Agarwal,

Enabling HPC Workflows on Clouds

Keith Jackson

John Bell

Low Order Models for Simulation of Ballistic Transport in Nanoscale Devices

Jonathan Carter, Leonid Oliker, Enhancing the Effectiveness of Manycore Chip Technologies for High-End Computing ............................................................... 28

John Shalf

Doron Rotem,

Energy Smart Disk-Based Mass Storage System

Ekow Otoo

James Sethian

Transforming Data: From Images to Models to Computational Input

John Shalf, Leonid Oliker, Holistic Approach to Energy Efficient Computing Architecture.... 31

Michael Wehner

Erich Strohmaier

Kesheng $\mathrm{Wu}$, Kamesh Madduri

Chao Yang, Stafano Marchesini

Reference Benchmarks for the Dwarfs (Algorithms)

Managing Petascale Data with Emerging Computer

Architectures

Computational Techniques for Non-crystalline X-ray

Diffraction Imaging 
Eoin Brodie

Michael Commer, Stefan Finsterle, Michael Kowalsky, Gregory Newman

Christer Jansson

Janet Jansson

Hui-Hai Liu

Curtis Oldenburg

Rohit Salve, William Dietrich, Stefan Finsterle

Margaret Torn
Uncovering the Mechanistic Basis for Soil Microbial

Community Response to Altered Precipitation Patterns

Enhanced Subsurface Fluid Characterization Using Joint Hydrological and Geophysical Imaging.....

Carbon Uptake and Partitioning in Plants and Algae

Microbiomics of Complex Microbial Communities in

Environmental Samples

Impact of Climate Change on Soil Water Dynamics in Arid Areas

CO2 as Cushion Gas for Compressed Air Energy Storage in Subsurface Reservoirs

Identifying and Predicting Climate Change Impacts on the Land-Based Components of the Water Cycle.

Biological Carbon Sequestration: Fundamental Research on Biological Carbon Capture and Soil Carbon Stabilization

Marco Battaglia, Peter Denes

Advanced Silicon Detectors for Future Short Pulse X-ray

Sources

Rafael Gomez-Sjoberg

Parallel Microfluidic Synthesizer: A Fully Automated Chemical

Evolution Platform for Novel Materials Discovery

Soren Prestemon

High-Temperature Superconductors for Compact X-ray FEL's.....

Environmental Energy Technologies Division

Vladimir Bazjanac

Katie Coughlin, David Fridley

Marc Fischer

Ashok Gadgil

John Kerr,

Venkat Srinivasan, Marca Doeff

David Lorenzetti, Michael Sohn

Randy Maddalena, Tom McKone, Michael Sohn

Phillip Price, Douglas Black
Embedded Engineering, Construction Materials, and HVAC Components in Modular Energy Systems Simulation

Physically-Based Accounting for Resource Use in Alternative Energy Pathways.

Toward a US Greenhouse Gas Information System (GHGIS) ...... 48

Developing a Fuel-Efficient Cook Stove for Haiti

Development of Novel Improved Capacitors for Pulse Power Applications

Probabilistic Optimization of Energy Systems in Buildings.

Relating Tissue Residues to Indoor Chemical Sources in a

Bayesian Framework: Synthesis of Chemistry,

Pharmacokinetics, and Biomarkers ...

Self-Tuning Building Energy Model 
Michael Wetter,

Wangda Zuo,

Alberto Sangiovanni-

Vincentelli

Genomics Division

Mark Biggin, $\quad$ Measurement of Protein/ DNA Binding in vitro and in vivo

Jian Jin

Using Single Molecule Approaches

Len Pennacchio, Assessing Epigenomic Approaches for Gene Enhancer

Axel Visel

Discovery.....

Life Sciences Division

Eleanor Blakely

Synchrotron-Based Microtomography for Functional Analysis

of Normal Tissue and Tumor Molecular Markers, and Their

Perturbation by Low-Dose Radiation Exposure

Luis Comolli

Linking Genomics, Proteomics and Ultrastructural

Characterization of Microbial Communities and Their Viruses.....

Jian-Hua Mao

Identification of Genetic Networks Controlling Susceptibility to

Radiation-Induced Carcinogenesis .

Cynthia McMurray

SPARKLE- A Fluorescence Energy Transfer (FRET)

Methodology for Visualization of Simultaneous and Reversible

Interactions

Antoine Snijders

Syngeneic Mouse Model for Breast Cancer Metastasis and

Organ Tropism.

John Tainer

Bio Energy Technologies and Science Integrated Efficiently (BETSIE)

Daojing Wang

Multinozzle Arrays for Single Cell Metabolomics

Claudia Wiese

Functional Characterization of NUCKS- a Potential Cancer

Susceptibility Locus Required for Recombination...

Materials Sciences Division.

Nitash Balsara,

Andy Minor,

Venkat Srinivasan

John Clarke,

Harmut Haeffner

Delia Milliron

Andrew Minor

Robert Schoenlein

Berend Smit

Matthew Tirrell

Jeffrey Urban

Feng Wang
Synthesis and Characterization of Self-Assembled Battery

Electrodes

Heating Rates of Planar Ion Traps for Quantum Information

Mixed Ionic and Electronic Transport in Solution-Processed Inorganic Nano-Composites.

In situ Electromechanical Probing in a Transmission Electron Microscope (TEM)

X-ray Studies of Charge-Order Dynamics in Complex Materials.. 69

Tuning the Self-Assembly of Membrane Proteins .......................... 70

Structured Charged Polymers

Understanding How Nanoscale Interfaces Modify Predicted Optical, Vibrational, and Electronic Properties

Integrated Optoelectonic Devices Based on Graphene 73 
Peidong Yang,

Ali Javey,

Junqiao $\mathrm{Wu}$

Yuegang Zhang
Design and Surface Properties of Semiconductor Nanowires

Surface-Selective Synthesis of Graphene Nanoribbons on

Nanowire Templates

75
76

Bolometric Detectors for the Neutrinoless Double-Beta Decay

Experiments........................................................................ 76

Heavy Element Mass Analysis and Detector Capabilities ............ 77

A $100 \mathrm{~km} 3$ Neutrino Detector for Ultra High Energy Neutrinos ... 78

Ultra-Sensitive Ge Detectors for Low-Background Physics

Experiments...................................................................... 79

Feng Yuan, $\quad$ Theoretical Study of Nucleon Structure ...................................... 80

Physical Biosciences Division.

Michelle Chang

Biological Methods for Synthesis of Iron-Based Nanomaterials ... 81

Daniel Fletcher

Engineering Environmental Sensitivity in an Artificial Cell 82

Héctor García Martín, Metafluxomics of a Phosphorus Removing Microbial

Aindrila Mukhopadhyay

Community

Jay Groves

Applications of Hybrid Live Cell- Synthetic Devices for Cancer Research

Nathan Hillson

Advances in Standardized, Scar-less, Sequence-Independent Cloning Methods.

Jan Liphardt

Light-Boosted Fermentation in the Yeast Saccharomyces cerevisiae.

Henrik Scheller

Engineering of Drought and Heat Tolerance in Bioenergy Crops..

Ahmet Yildiz

Control of Intraflagellar Transport in Chlamydomonas Cells......... 88

Peter Zwart, Development of Reusable Software Modules for the Analyses

Alexander Hexemer, of Small Angle Scattering Data

Paul Adams

Physics Division

Murdock Gilchriese, Carl Haber

Richard Muller

Yasnori Nomura

Natalie Roe, Peter Denes, Steve Holland

George Smoot, Alexie Leauthaud

Nikhil Padmanabhan, Martin White, David Schlegel
Development of Multi-Modular Assemblies with Reduced Material and Services for Tracking at Future Colliders ................ 90

Study of History of Global Surface Temperatures ....................... 91

Theoretical Studies of Dark Matter Beyond the Standard Model... 92

R\&D for Fast, Low-Noise CCD Readout and Single Photon Detection Capability

Decoding Dark Energy with Weak Gravitational Lensing

Calibrating Baryon Acoustic Oscillations for Future Dark Energy Experiments. 


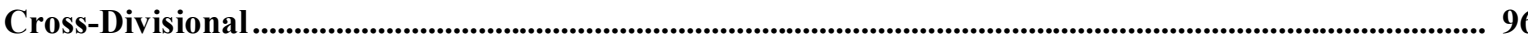

Manfred Auer,

Integrated Tools in Multiscale Imaging .....

96

Jan Liphardt,

Phil Hugenholtz,

Terumi Kohwi-

Shigematsu

Cheryl Kerfeld,

Predictive High-Throuput Assembly of Synthetic Biological

Adam Arkin,

Systems: From Gene Expression to Carbon Sequestration

97

Eddy Rubin

Delia Milliron,

Jeffrey Urban,

Multifunctional Window Coatings for High-Performance

Yuegang Zhang,

Buildings.

André Anders,

Thomas Richardson

Lee Schroeder et al

Coupled Process Models, Separations, Licensing/Monitoring

for Advanced Nuclear Fuel Cycles.

99

Publications List 


\section{Introduction}

The Ernest Orlando Lawrence Berkeley National Laboratory (Berkeley Lab or LBNL) is a multi-program national research facility operated by the University of California for the Department of Energy (DOE). As an integral element of DOE's National Laboratory System, Berkeley Lab supports DOE's missions in fundamental science, energy resources, and environmental quality. Berkeley Lab programs advance four distinct goals for $\mathrm{DOE}$ and the nation:

- To perform leading multidisciplinary research in the computing sciences, physical sciences, energy sciences, biosciences, and general sciences in a manner that ensures employee and public safety and protection of the environment.

- To develop and operate unique national experimental facilities for qualified investigators.

- To educate and train future generations of scientists and engineers to promote national science and education goals.

- To transfer knowledge and technological innovations and to foster productive relationships among Berkeley Lab's research programs, universities, and industry in order to promote national economic competitiveness.

Berkeley Lab's research and the Laboratory Directed Research and Development (LDRD) program support DOE's Strategic Themes that are codified in DOE's 2006 Strategic Plan (DOE/CF-0010), with a primary focus on Scientific Discovery and Innovation. For that strategic theme, the Fiscal Year (FY) 2010 LDRD projects support each one of the three goals through multiple strategies described in the plan. In addition, LDRD efforts support the four goals of Energy Security, the two goals of Environmental Responsibility, and Nuclear Security (unclassified fundamental research that supports stockpile safety and nonproliferation programs). The LDRD program supports Office of Science strategic plans, including the 20year Scientific Facilities Plan and the Office of Science Strategic Plan. The research also supports the strategic directions periodically under consideration and review by the Office of Science Program Offices, such as LDRD projects germane to new research facility concepts and new fundamental science directions.

Berkeley Lab LDRD program also play an important role in leveraging DOE capabilities for national needs. The fundamental scientific research and development conducted in the program advances the skills and technologies of importance to our Work For Others (WFO) sponsors. Among many directions, these include a broad range of health-related science and technology of interest to the National Institutes of Health, breast cancer and accelerator research supported by the Department of Defense, detector technologies that should be useful to the Department of Homeland Security, and particle detection that will be valuable to the Environmental Protection Agency.

The Berkeley Lab Laboratory Directed Research and Development Program FY2010 report is compiled from annual reports submitted by principal investigators following the close of the fiscal year. This report describes the supported projects and summarizes their accomplishments. It constitutes a part of the LDRD program planning and documentation process that includes an annual planning cycle, project selection, implementation, and review.

The Berkeley Lab LDRD program is a critical tool for directing the Laboratory's forefront scientific research capabilities toward vital, excellent, and emerging scientific challenges. The program provides the resources for Berkeley Lab scientists to make rapid and significant contributions to critical national science and technology problems. The LDRD program also advances Berkeley Lab's core competencies, foundations, and scientific capability, and permits exploration of exciting new opportunities. All projects are work in forefront areas of science and technology. Areas eligible for support include the following:

- Advanced study of hypotheses, concepts, or innovative approaches to scientific or technical problems;

- Experiments and analyses directed toward "proof of principle" or early determination of the utility of new scientific ideas, technical concepts, or devices; and

- Conception and preliminary technical analyses of experimental facilities or devices.

The LDRD program supports Berkeley Lab's mission in many ways. First, because LDRD funds can be allocated within a relatively short time frame, Berkeley Lab researchers can support the mission of the Department of Energy (DOE) and serve the needs of the nation by quickly responding to forefront scientific problems. Second, LDRD enables Berkeley Lab to attract and retain highly qualified scientists and to support their efforts to carry out worldleading research. In addition, the LDRD program also supports new projects that involve graduate students and postdoctoral fellows, thus contributing to the education mission of Berkeley Lab.

Berkeley Lab has a formal process for allocating funds for the LDRD program. The process relies on individual 
scientific investigators and the scientific leadership of Berkeley Lab to identify opportunities that will contribute to scientific and institutional goals. The process is also designed to maintain compliance with DOE Orders, in particular DOE Order 413.2B (dated April 19, 2006). From year to year, the distribution of funds among the scientific program areas changes. This flexibility optimizes Berkeley Lab's ability to respond to opportunities.

Berkeley Lab LDRD policy and program decisions are the responsibility of the Laboratory Director. The Director has assigned general programmatic oversight responsibility to the Deputy Director, with administration and reporting on the LDRD program supported by that office. LDRD accounting procedures and financial management are consistent with the Laboratory's accounting principles and stipulations under the contract between the University of California and the Department of Energy, with accounting maintained through the Laboratory's Chief Financial Officer.

In FY2010, Berkeley Lab was authorized by DOE to establish a funding ceiling for the LDRD program of $\$ 22.7 \mathrm{M}$ including General \& Administrative (G\&A) overhead, which equated to $\sim 3.5 \%$ of Berkeley Lab's FY2010 projected operating and capital equipment budgets. This funding level was provided to develop new scientific ideas and opportunities and allow the Berkeley Lab Director an opportunity to initiate new directions. Budget constraints limited available resources, however, so a little more than $\$ 20.6 \mathrm{M}$ was expended for operating expenses (3.00\% of actual Berkeley Lab FY2010 operating and equipment costs excluding ARRA funding).

In FY2010, scientists submitted 202 proposals, requesting about $\$ 41.1 \mathrm{M}$ in funding prior to assessing laboratory overhead. Ninety nine projects were funded, with awards ranging from $\$ 52 \mathrm{~K}$ to $\$ 997 \mathrm{~K}$. These projects are identified in the Table of Contents. 


\title{
Accelerator and Fusion Research Division
}

LB10032

\author{
Plasma-Assisted High Rate Deposition Concept for Energy Applications \\ Principal Investigator: André Anders
}

\section{Project Description}

The purpose of this project is to significantly enhance (by a factor 2 or greater) the deposition rate of coatings used in energy-related devices such as solar panels, solar concentrators, photo-catalytic surfaces, and energy-efficient windows (low-emissivity, solar control, and electrochromic). Specially, we consider low-cost (indium-free) high quality transparent conducting oxide films. Such films are currently produced by reactive sputtering, a well established but slow and costly process.

It is straight-forward to increase the absolute deposition rate by simply increasing the power to the sputtering system, however, this approach runs into serious limitations such as overheating of the apparatus, followed by melting of the target and damage to the magnets, and switching to the undesired arcing mode. In this project we explore an innovative way of enhancing the absolute and relative (power-normalized) deposition rates while maintaining the features of plasma assistance to film growth: we investigate the opportunities of hot target sputtering with controlled temperature and plasma pulsing. Additionally, we address the issues of too energetic ion bombardment, which is detrimental to the quality of crystalline films.

Accomplishments

Hot target sputtering has been tested for a number of target materials and found to strongly correlate with the target's vapor pressure at elevated temperature. This allows us to combine sputtering and evaporation with plasma assistance, hence combining a high rate process with a high quality process. Socalled ion filtering by plasma lensing is another concept being pursued in this context (see figure). Patent disclosures have been filed, and initial reports have been given at international conferences

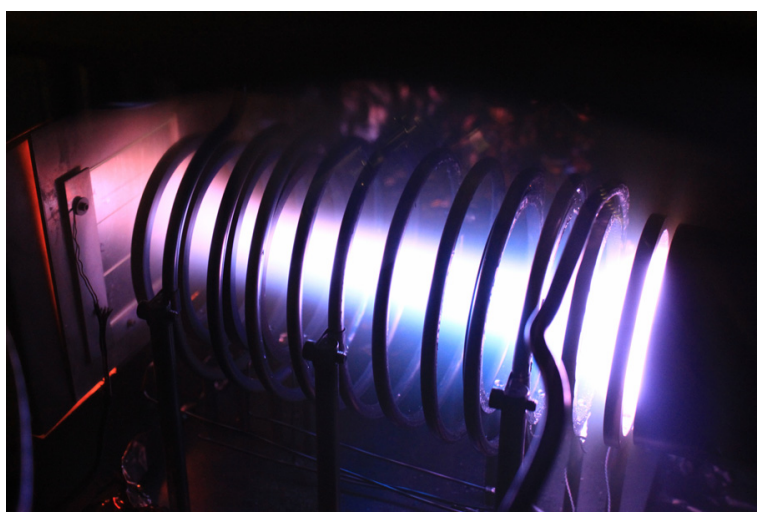

Figure: Photograph of a plasma lens developed for high rate sputter-deposition of oxide films. such as the International Conference on Coatings of Glass and Plastics. The preliminary results of the first year are the basis for designing and manufacturing of a novel hybrid source that specifically aims to make lowcost, high quality doped zinc oxide films, but it could be used for other coatings as well. A rate increase by a factor 5-10 compared to conventional magnetron sputtering of such films seems feasible. 
Ion Beam Driven Fusion-Fission Hybrids

Principal Investigators: Bernhard Ludewigt and Peter Seidl

\section{Project Description}

In this project we seek to assess the potential of ion-beam driven fusion-fission reactor hybrids and accelerator-driven systems. Both approaches employ subcritical fission blankets that are driven by neutrons from either a heavy-ion beam driven inertial fusion system or a highenergy proton beam driven spallation source.

Parametric studies on a variety of thorium-fueled, radially-zoned liquid-salt cooled pebble bed and other subcritical fission blankets are performed to determine the maximum energy multiplication a blanket could be designed to produce when driven by a spallation neutron source, as well as the attainable burnup, i.e., the fraction of the fuel loaded that could be fissioned without fuel reprocessing. The question addressed is weather an accelerator-driven system can be designed that commercially uses thorium or uranium without the need for enrichment or fuel reprocessing, both of which are proliferation concerns.

Ion beam accelerator options capable of providing the high average beam power and high power efficiency needed are investigated to identify R\&D needs and possible paths forward.

Potential benefits of an ion-beam driven fusion-fission hybrid over a heavy-ion fusion reactor are evaluated. In a hybrid the heavy ion fusion system could drive an uranium/thorium-fueled, Flibe-cooled pebble-bed blanket. Questions being addressed include whether the use of the blanket reduces the radiation damage to the first structural wall so that it can last for the entire life of the reactor, whether sufficient energy multiplication can be reached, and whether ionbeam driver requirements can be lowered.

\section{Accomplishments}

Several blanket designs were studied for accelerator-driven systems. The design variables considered in the parametric study of the thorium-fueled, liquid-salt cooled pebble bed blanket included the thorium-to-carbon atom ratio in the pebbles, the discharge burnup and the pebbles shuffling scheme. The maximum energy multiplication was found to be 1.6 with a burnup of $20 \%$. Alternative blanket designs using depleted uranium were found to attain higher, but still insufficient energy multiplication of $\sim 3$. Also explored was an innovative concept for using an accelerator-driven system to double the energy extracted from "breed-and-burn" reactor fuel. These reactors, currently under development, are designed to burn up to $20 \%$ of the uranium without having to reprocess the fuel. The used fuel could be double clad and reused as a blanket in an accelerator-driven system. With doubling of the burnup to $40 \%$ and an energy multiplication larger than 10 the concept has promise.

Proton accelerators for the spallation neutron sources driving the subcritical assemblies must deliver a beam power on the order of $10 \mathrm{MW}$ at energies in the 0.5 to $1 \mathrm{GeV}$ range for producing more than $10^{18} \mathrm{n} / \mathrm{s}$. Possible accelerator choices are room-temperature or superconducting cyclotrons and superconducting linacs. Maximizing power efficiencies, currently $10-15 \%$ for comparable accelerators, will be important.

We modeled a fusion-fission hybrid consisting of a pebble-bed type blanket fueled with depleted uranium and a heavy-ion beam driven inertial fusion neutron source. In our initial analysis the helium buildup in the first wall is the limiting factor; it dictates using a liquid Flibe wall of nearly $80 \mathrm{~cm}$ in thickness, which reduces the power generated by the fission blanket to about $20 \%$ of total in our not optimized design. 
Novel Accelerator \& Engineering Strategies for Ion Beam Cancer Therapy Principal Investigator(s): David Robin

\section{Project Description}

Ion-beam cancer therapy (IBCT) is the use of ion beams, mostly proton and carbon beams, to treat cancer tumors. IBCT has achieved remarkable clinical success worldwide, and research interest is expanding, but in many cases is based on outdated technologies. Improvement in technology has the potential to benefit patients, hospitals, and society through more-effective treatment modalities; smaller, less costly accelerators; and better beam delivery, and dosimetry systems. The purpose of this project was to identify areas where LBNL has the capability to improve IBCT technology.

We plan to survey the status and this multi-disciplinary field and identify current technical opportunities. We have already identified areas of research needed for a more cost-effective and technical optimal ion beam therapy capability. We will concentrate on the research areas most needed and best suited to our capabilities, involving engineering studies, scoping of experiments, and computer calculations. There are two areas we decided to ultimately explore in this project: advanced superconducting beam delivery systems and real time detection mechanism for real time imaging and dose distribution.

\section{Accomplishments}

For an optimal 3D conformation of the tumor volume, an optimal solution is the installation of an "isocentric gantry" with 360-degree rotation to the incident beam. Present gantries are large and heavy. In particular carbon beams have three times the magnetic rigidity compared with proton beams thus requiring significantly larger gantries. While most proton facilities are being built with gantries, there exists only one carbon gantry in the world (in Heidelberg) whose size is nearly double that of a proton gantry and at 660 tons is 5 times heavier than proton gantries. Much of this is due to the size of a single magnet, a final $90^{\circ}$ bend, which in the case of the Heidelberg gantry weights $\sim 100$ tons. Our most significant accomplishment is in the development of compact superconducting gantries for carbon ion beams.

With the goal of reducing the size of a carbon gantry down to the level of a proton gantry, we have been exploring novel high field superconducting magnets as gantries with fields three times larger than can be achieved with normal conducting magnets. In particular we have been exploring a particular design using a tilted solenoid pair. What we found is that such a superconducting super conducting magnet has the promise of reducing the weight of the magnet by nearly an order of magnitude compared to the normal conducting Heidelberg magnet 
Experimental accelerator R\&D toward a future light source at LBNL Principal Investigator: Fernando Sannibale.

Project Description.

This LDRD project is devoted to the design, development and construction of hardware systems that critically impact the performance and cost of a soft X-Ray Free Electron Laser (FEL) light source. In particular, the project targets the development of a novel electron photogun capable of generating the high brightness electron beams required by the operation of a high repetition (MHz class) X-Ray FEL such as the Next Generation Light Source (NGLS) that LBNL is proposing. The final performance of such sources ultimately depends on the quality of the electron beam source, and existing guns fail to satisfy the beam quality and high repetition rate requirements simultaneously. For this reason, the development of the new scheme conceived at LBNL represents a necessary and critical step towards the development of the LBNL light source. The LDRD funds are mainly applied to the design and fabrication of the gun and of its sub-systems, and in particular of the core of the source, the $187 \mathrm{MHz}$ radio frequency (RF) cavity designed to operate in continuous wave (CW) mode, and capable of achieving $20 \mathrm{MV} / \mathrm{m}$ accelerating field at the cathode (as required for obtaining the necessary beam quality), and vacuum pressures in the low $10^{-11}$ Torr decade. Such low pressure values are necessary to operate the high quantum efficiency cathodes capable of generating the required electron charge (up to a $\mathrm{nC}$ ) with existing laser technology.

Accomplishments.

This FY10 LDRD is the second year continuation for the project. During FY09, the following main goals were achieved: i) the final design was completed and the fabrication of the $187 \mathrm{MHz}$ cavity was initiated; ii) the specification for the $120 \mathrm{~kW}, 187 \mathrm{MHz}$ RF power source required to operate the cavity at full operating gradient were defined.

In the FY10 second year LDRD, the funds allowed to:

- Complete the fabrication of the $187 \mathrm{MHz}$ cavity.

- Successfully perform the low power RF test and the initial vacuum test of the cavity.

- Assign the bid and proceed with the acquisition of the $120 \mathrm{~kW} 187 \mathrm{MHz}$ RF source for the cavity. The system is now fully built and under commissioning at the factory.

- Develop and construct in collaboration with Lawrence Livermore National Laboratory the laser system for generating the electron photoemission from the gun photo-cathode. The system is now completed and under commissioning at LBNL.

- Install the cavity inside the Beam Test Facility (BTF).

- To continue with the preparation of the test area for receiving all the gun sub-systems.

- Complete the design and construction of the RF couplers to supply RF power to the cavity.

- Complete the design of the RF distribution system.

During FY10, we were successful in receiving funding for this area of R\&D from the DOE BES Accelerator and Detector R\&D Program, and thus we terminated support from the LDRD for these activities. The remaining LDRD funds were re-addressed towards the following two new technical questions that are also critical issues for the NGLS.

- Design and prototyping for a fast pulser, and development of design concepts for a kicker, required for the beam spreader of the NGLS. A pulser prototype is presently under test.

- Design studies for a high repetition-rate and high-brightness injector for NGLS, based on the $187 \mathrm{MHz}$ gun. The beam dynamics studies are now in advanced phase with quite promising results. 
Quantum information science with integrated color centers in diamond Principal Investigator: Thomas Schenkel, AFRD, LBNL

\section{Project description:}

Color centers in diamond, like the nitrogen-vacancy center, exhibit amazing coherence properties, with spin lifetimes approaching milliseconds at room temperature. Color centers promise to revolutionize quantum information technology and our fundamental understanding of quantum coherence when they can be integrated into devices. To date, studies have been limited to uncontrolled, randomly distributed centers, and the coherence properties of many promising centers have not been characterized for lack of efficient center formation. We are forming arrays of color centers with unprecedented spatial resolution (few $\mathrm{nm}$ ) by ion implantation with scanning probe alignment and integrate center arrays into opto-electronic devices. We are also developing processes for efficient center formation and characterize coherence properties of new center systems.

\section{Accomplishments:}

Our unique setup for ion implantation with scanning probe alignment allows nondestructive imaging, alignment and ion placement with nanometer resolution and is ideally suited for the formation of color center arrays in diamond samples (which are usually small and rare). Single ion placement is enabled by the sensing of induced charge from single ion impacts, and we have formed first devices with tungsten electrodes on diamond for demonstration of single ion detection in diamonds. Samples are selected from commercial supplies of synthetic diamonds with ultra-low nitrogen content. We make use of the Imaging Facility at the Molecular Foundry for array characterization and collaborate very closely with Prof. Awschalom (UCSB) on the mapping and control of coherence properties using confocal laser spectro-microscopy, and optically detected magnetic resonance.

In our third year of this LDRD we refined processing recipes for more reliable color center formation by co-implantation of hydrogen, helium and carbon ions following nitrogen ion formation. We characterized and simulated range profiles and studied photoluminescence and coherence properties of NV centers in arrays. These studies lay the foundation for future integration of high quality color centers in diamond for quantum information processing and novel imaging applications. 


\section{Experimental demonstration of a laser-plasma-accelerator driven free-electron laser Principle Investigator(s): Csaba Toth, Carl Schroeder, William Fawley, Kem Robinson}

\section{Project Description}

The goal of this project is the demonstration and development of a free-electron laser (FEL) driven by the laser wakefield accelerator (LWFA) currently operating in AFRD's laser-plasma accelerator facility, the LOASIS Laboratory. The LDRD project consists of two stages: (1) The LWFA electron beam will be coupled to a $2.18 \mathrm{~cm}$-period undulator and the synchrotron radiation from three $(3 \times 22=66$-period $)$ undulator sections will be used to diagnose and characterize the LWFA electron beam. The intrinsic synchronization between the laser, electron beam, and radiation will be demonstrated. (2) Gain will be demonstrated via self-amplified spontaneous emission (SASE) in the XUV wavelength range. Fluxes on the order of $10^{13}$ photons/pulse are anticipated at saturation due to the high current $(>10 \mathrm{kA})$ of the LWFA beams. The LWFA-FEL will constitute a new class of compact laser-driven FEL [in which the conventional accelerator (10-100 m length) is replaced by a GeV-class laser-plasma accelerator $($ several $\mathrm{cm})$ ] producing ultra-short $(<50 \mathrm{fs})$ XUV pulses with high peak flux.

\section{Accomplishments}

We have achieved important results in five areas required for experimental demonstration of a LWFA-driven FEL:

(1) Significantly improved the electron beam quality of the LWFA via installing, monitoring and fine-tuning an optical prepulse contrast filter, and a laser beam pointing stabilization system in the laser beam (which generates the e-beam). Experiments performed with gas-jet+capillary integrated target design provided greatly improved e-beam energy stability and tuneability.

(2) Wavefront and reflectivity measurements performed both at waterjet and tape-drive test stands show sufficient flatness of laser produced plasma layers for laser reflection and revealed optimal laser intensity range for high reflectivity. These plasma mirrors will provide compact methods to separate the laser and electron beam.

(3) The THUNDER undulator from Boeing is fully characterized by Hall-probe magnetic measurements at LBNL. The undulator and the alignment structures are installed in the fully renovated Bldg. 71 Cave-A (May 2009). The radiation interlock protection system has been installed, and systematic e-beam operation and characterization are ongoing since Sep 2009. Installation of quadrupole magnets for e-beam refocusing and steering allowed precise aiming of the e-beam toward the undulator input (May 2010). RF cavity based beam-positioning monitors (BPMs) provided vital feedback during these experiments (since Aug 2010).

(4) XUV grating spectrometer capable of measuring the fundamental and harmonic (upto $3^{\text {rd }}$ ) undulator radiation has been designed, built and installed. Successful calibration tests were performed at ALS (Spring 2010), and the system is ready for measurements in Cave-A.

(5) The expected spontaneous radiation emission was calculated using the code SPECTRA. Sensitivity to electron beam energy spread, emittance, and beam focusing (beam spot and divergence evolution through the undulator) were investigated. Matching the electron beam to the THUNDER undulator (60 micron beam radius in the undulator) using compact, high-field quadrupole magnets $(540 \mathrm{~T} / \mathrm{m})$ was examined using the particle tracking code GPT, assuming laser-plasma accelerator beam parameters at the exit of the plasma. Output FEL radiation was modeled using the FEL code GINGER. Higher energy electron beams were considered to generate soft $\mathrm{x}$-ray $(1 \mathrm{~nm})$ radiation. 


\section{Lorentz Compaction of Scales for Ultra-Efficient Simulation \\ of Advanced Accelerators (and Other Systems). \\ Principal Investigators: Jean-Luc Vay, William Fawley}

\section{Project Description}

The purpose of this project is to speed-up by orders of magnitude a class of first-principle computer simulations of complex physical problems, by recasting them into a Lorentz boosted frame of reference. Design and understanding of modern experimental devices relies increasingly upon detailed computer simulation, but despite the progress in computer power and algorithms, some problems remain extraordinarily difficult to model accurately. One such problem is laser wakefield acceleration (LWFA): modeling 10-GeV stages in 1D in the lab frame demanded as much as 5,000 processor-hours, indicating 3D modeling would be impractical.

During the course of this project, we will: (1) demonstrate, with actual 2-D or 3-D computer simulations of problems relevant to LWFA, electron clouds (EC) and free electron lasers (FEL) physics problems, orders of magnitude speed-up from a calculation in the laboratory frame to a calculation in the optimum Lorentz frame of reference; (2) for each targeted application, determine the range of validity for the Lorentz-boosted frame simulation results; (3) explore other scientific applications.

\section{Accomplishments}

In year 3, our most significant achievement has been the first multidimensional simulations of LWFA stages in the $10 \mathrm{GeV}-1 \mathrm{TeV}$ range for stages which efficiently use (or 'deplete') the laser energy [1-4]. Efficient depletion of the laser energy to the wake and particle bunch is required to accurately model experiments and for applications to obtain useful beam energy and charge from such accelerators, but presents problems that limit other techniques. It was demonstrated that the boosted frame technique accurately captures these effects, allowing for the first time modeling of $10 \mathrm{GeV}$ stages for the BELLA laser and modules for a conceptual future laser-plasma collider at full scale, including both depletion and loading of the wake by the particle beam [5]. It was further used to verify for the first time the performance of LWFAs into the $\mathrm{TeV}$ range (without loading). This calculation has been possible thanks to up-to over 1 million times speedup in runtime by recasting the simulation in the frame of the wakefield. This, in turn, was enabled by the successful mitigation of a numerical instability that had plagued previous work by us and others, thanks to: (1) a key observation that a second relativistic effect (hyperbolic rotation) permits a particular boost velocity that optimizes effectiveness [1], (2) several numerical advances including implementation of a new efficient digital filtering algorithm and a novel electromagnetic solver with tunable coefficients in the PIC code Warp, (3) discovery of a "magical" time step at which the instability rate is greatly reduced [6]. Another essential component to the success was a new versatile, yet simple, laser injection routine that was implemented, avoiding an explosion of the simulation grid transverse size due to the shortening of the rayleigh length in the boosted frame [2-3]. The method is now applied to the design of $10 \mathrm{GeV}$ stages for the BELLA project that is under construction at LBNL [5].

We successfully demonstrated the boosted frame method on free electron laser (extending to 3D in year 3) and coherent synchrotron radiation on proof of principle problems [7-8]. The method offers efficient direct 3D self-consistent simulations from first principle, giving access to some important physics that is ignored by other reduced models, enabling future simulations of increased detail and accuracy. 


\section{Linac Driver and X-Ray FELs for Next Generation Light Sources \\ Principal Investigator: Marco Venturini}

\section{Project Description}

The purpose of the project is to investigate advanced concepts applicable to a nextgeneration free electron laser (FEL)-based light source that could be implemented at LBNL, meeting the goal of delivering high-energy, high-quality electron beams at a high rate for production of soft X-ray pulses. Expanding on work carried out in recent years at LBNL and elsewhere, we intend to explore novel techniques for beam control and manipulations while evaluating conceptual designs for the accelerators subsystems and lasing schemes capable of generating the desired radiation output.

\section{Accomplishments}

Our work has followed two main lines of activities supporting the design of the FEL beamlines and the design of an accelerator driver capable of delivering beams with the required characteristics to the FEL. We have placed emphasis on both the exploration of novel and/or untested (but promising) concepts and the development of machine configurations.

Among the most noteworthy accomplishments we would like to mention: $i$ ) the development of a novel scheme for the generation of two-color attosecond X-ray pulses based on the Echo Enhanced High Harmonic (EEHG) generation technique and its implementation in a $2.4 \mathrm{GeV}$ energy machine. ii) The identification of a novel longitudinal bunch compression scheme based on transverse-longitudinal emittance exchange that does not require imparting an energy chirp to the beam. iii) The proposal to support EEHG using FEL oscillators to generate the laser pulses needed to manipulate and seed the beam for lasing at high harmonics. $i v$ ) The proposal of a new FEL seeding method based on modulation compression performed while compressing the beam through a conventional magnetic chicane.

We consolidated the design lattice for a $2.4 \mathrm{GeV}$ linac that was developed previously and explored trade-offs in the machine and electron-beam parameter spaces with the intent to minimize cost while preserving performance. An outcome of these studies was the development of a point design for a $1.8 \mathrm{GeV}$ energy accelerator and a detailed design for 3 possible distinct FEL beamlines (one SASE, the other two seeded) to be supported by such a machine. Additional activities have included, but have not been limited to, the examination of "single-spike" SASE operations for a wide range of values of electron bunch and undulator parameters; evaluation of High Harmonic Generation seeding options and examination of a variety of schemes, including a two-stage HGHG beamline, a single-stage HGHG beamline, and "afterburner" configurations; development of new numerical tools and analytical models for studying the beam dynamics (e.g. new optimization routines for beam matching in the linac; analysis of microbunching instability through rf and magnetic compression); theoretical/modeling support to the ECHO 7 experiment at SLAC. 


\section{Advanced Light Source Division}

Optimization of Flux Pinning in Type II Superconductor Based Magnets for Soft X-ray Scattering Applications

Principal Investigator(s): Elke Arenholz, Soren O. Prestemon

Project Description

Type II superconductors can trap magnetic flux and retain it after field removal if cooled in an external field through their transition temperature. We proposed to use this approach to provide background field near $5 \mathrm{~T}$ in soft $\mathrm{x}$ ray magnetic scattering experiments on novel magnetic materials.

\section{Accomplishments}

It was the goal of the previous FY2008 and FY2009 LDRDs and the FY2010 continuation to demonstrate that flux pinning in type II superconductors is a viable and powerful method to provide magnetic background fields near $5 \mathrm{~T}$ for soft x-ray scattering experiments.

With funding from the FY2008/2009 LDRDs a test and characterization set up for the detailed determination of flux pinning in the YBCO discs was designed and built. We identified Y-Ba-Cu-O (YBCO) discs (20 mm diameter, $8 \mathrm{~mm}$ thick) provided by R. Weinstein, University of Houston, as most suitable materials for our soft x-ray scattering applications since up to $80 \%$ of the field applied during field cooling is reliably pinned in the YBCO discs by defects after removal of the external field at 5K. Moreover, flux creep, i.e. the loss of pinned flux due to thermal fluctuations, can be completely eliminated by lowering the temperature of the YBCO disc in zero field after the field cooling process leading to very stable experimental conditions. The field distribution near the YBCO surface after the temperature field cycle as well as its dependence on cooling field and temperature has been studied in detail.

Moreover, we designed and built a scattering chamber that uses flux pinning for soft x ray scattering experiments. The set up is shown in Figure1 (a). A variable temperature sample

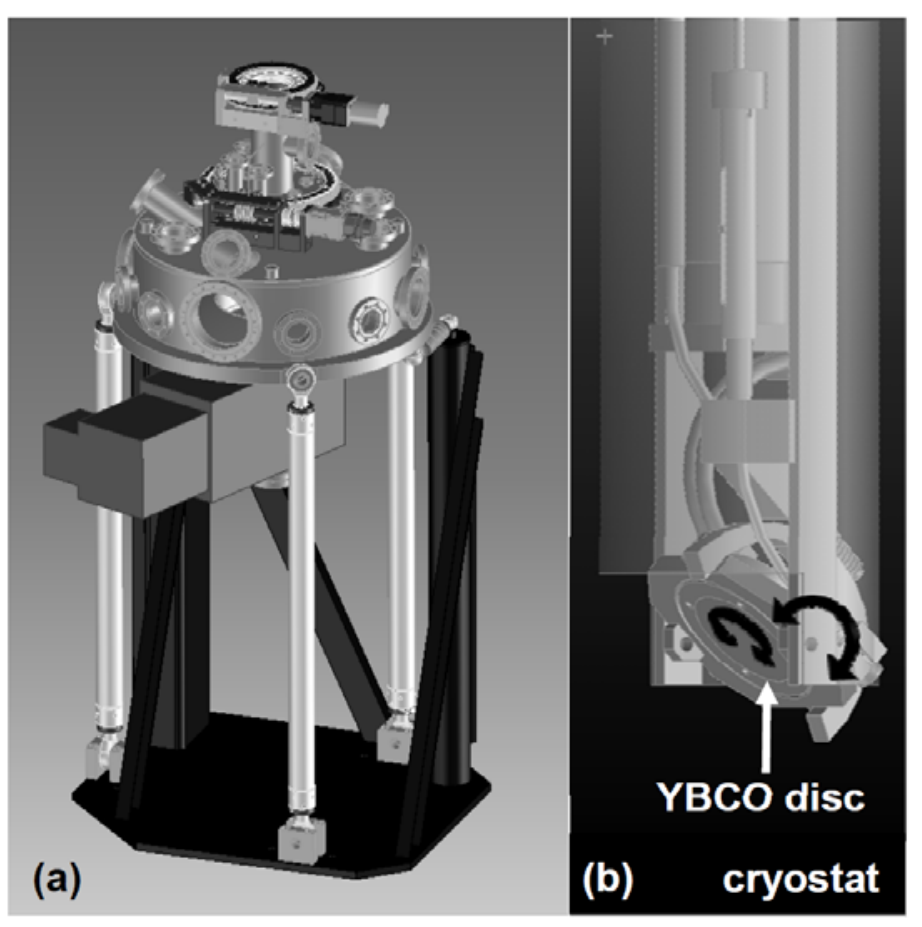
holder that retains the YBCO disc as well as the sample is displayed in Figure 1(b). The system is being commissioined and will be used for first scattering experiments at the Advanced Light source shortly.

Figure 1: Design of (a) soft x ray scattering chamber and (b) optimized sample holder employing the flux pining approach developed within the scope of this LDRD. 


\section{Soft X-ray Scattering As a New Probe of Polymer Systems}

Alexander Hexemer, Howard Padmore, Zahid Hussain, Jeff Kortright, Nitash Balsara, Rachel Segalman, Ting Xu, Ed Kramer, Sunil Sinha, Harald Ade, Rex Hjelm, Lynn Loo, Thomas Russell

\section{Project Description}

This project is to apply soft-x-ray scattering to polymer and related soft and hard condensed matter. The ultimate outcome will be to make a scientific case for a dedicated ALS beamline. Many polymer applications are envisioned that rely on heterogeneous morphology in block copolymers to yield specific functional properties, such as organic light-emitting diodes and photovoltaics. Materials and device development in many of these areas is limited by generally applicable probes of polymer morphology with high chemical sensitivity and special resolution. Resonant soft x-ray scattering (RSoXS) should fill the need for a high resolution chemical probe with interfacial sensitivity.

RSoXS is a new X-ray probe that can be used to analyze polymers and related hard and soft condensed matter. It combines the conventional hard $\mathrm{x}$-ray scattering with the unique elemental and chemical sensitivities by using soft x-ray near the absorption edge of the constituent materials, which can provide enhanced and tunable contrast between different components. It simultaneously provides chemical information, spatial information, and information about interfaces. By using this new technique, researchers will be able to understand how different structures can generate different functional properties in these materials. The ability to understand these structure-function relationships is crucial if researchers are to develop a new generation of materials and devices, and progress is currently limited by a lack of high-resolution analytical probes. RSoXS should fill the need for a high-resolution spatial probe based on chemical composition.

\section{Accomplishments}

During FY10, we have constructed a dedicated and optimized resonant soft x-ray scattering endstation at BL 11.0.1. Accommodating two endstations, PEEM3 (BL 11.0.1.1) and RSoXS (BL 11.0.1.2), BL11.0.1 is a state of art elliptically polarized undulator beamline that covers the energy range of 200-1300 eV. Combining high flux, high energy resolution and full polarization control, the soft x-ray scattering endstation accommodates a large variety of thin film samples and scattering geometries including transmission, specular and off-specular reflection, and grazing incidence geometries that allows users to study both laterally- and depth-resolved structure in ultrathin polymer films with essentially any form of chemical heterogeneity and degree of order with nanometer resolution. At the end of the projecthe beamline started to function as a regular user beamline utilizing $30 \%$ of the total beamtime for BL 11.0.1.

Additional researches from different scientific areas including energy research, material science, biological science, and environmental science have been conducted at BL 11.0.1.2 over the past year. Among which, our recent research work on organic photovoltaic has been selected in the most recent ALS science highlight, "Structure of All-Polymer Solar Cells Impedes Efficiency". 
Long-range Ordering of Block Copolymers on Faceted Silicon

Principle Investigator(s): Alexander Hexemer, Howard Padmore, Ting Xu, Thomas P.

Russell

\section{Project Description}

The purpose of this project is to generate an ultradense array of addressable nanoscopic elements over macroscopic length scale by using ordered block copolymers (BCPs). The self-assembly of block copolymer, two chemically dissimilar polymers covalently bond together, has become a promising route to generate templates and scaffolds for the fabrication of nanostructured materials. In general, BCPs in thin films self-assemble into grains, tens of microns in size, of laterally ordered nanoscopic microdomains. We have shown that the surface reconstruction of single crystalline wafers, cut along specific crystallographic planes, can be used to generate nanoscopic surface facets that can guide the self-assembly of BCPs into a highly ordered, single grain arrays of nanoscopic elements with a well-defined orientation over large areas. However, perfection in the ordering has not yet been achieved. Therefore, for guiding the selfassembly of BCPs microdomains in single grain, well-modulated silicon faceted surface for BCPs self-assembly was fabricated by chemical etching.

In order to quantitatively determine the crucial parameters for the perfection of $\mathrm{BCP}$ ordering on faceted surface, we will further develop grazing incidence small angle X-ray scattering (GISAXS). Currently GISAXS is only technique to examine nanoscopic elements in large area because of its great sensitivity. These GISAXS results will also be combined with computer simulation to understand how faceted surface function.

\section{Achievements}

We have succeeded in generating defect-free faceted silicon substrates over macroscopic distance. We use the combination of photolithography and wet anisotropic etching techniques to modulate precisely pitch distance of silicon saw-tooth varying from $100 \mathrm{~nm}$ to sub-microns although aspect ratio of pitch distance versus amplitude is fixed by 10 , which may be ideal for perfection of BCP ordering. We expect that adjusting the aspect ratio of pitch distance versus amplitude will be helpful to obtain further more information to understand the mechanism.

Lateral long-range ordering of BCP microdomians was successfully achieved on faceted silicon substrates by a solvent-annealing process that can induce enough mobility of polymer chains so that BCP microdomians can phase separate. Different amphiphilic BCPs such as polystyrene-block-poly(ethylene oxide) and polystyrene-blockpoly(vinylpyridine) copolymers were spin-coated on the substreates. Solvent-annealing method that we have established in this LDRD project can generate BCP microdomains oriented normal as well as parallel to the surface. By controlling solvent-annealing condition, two different orientations of BCP microdomains in single grain over the entire wafer surface $\left(\mathrm{cm}^{2}\right.$ in area) have been achieved independently.

So far, it has been impossible to generate addressable arrays of nanoscopic elements over macroscopic length scales by any means. Therefore, producing macroscopic arrays of addressable nanoscopic elements has the potential for ultradense, highly efficient photovoltaic devices and, also, ultradense, addressable media. 


\section{Search for a Permanent Electron Electric Dipole Moment (EDM) \\ Principal Investigator: David Kilcoyne}

Project Description:

The purpose of this project is to develop the technology needed to increase signal intensity, improve systematics suppression, and reduce magnetic field noise as the start of a new experiment to discover or rule out a permanent electric dipole moment (EDM) of the electron as small as $2 \times 10^{-50} \mathrm{C}-\mathrm{m}$ (a factor of 100 below the present limit).

Since 1990, all improvements to the electron EDM upper limit have come from a series of thermal atomic beam experiments at LBNL, begun in 1987 by Harvey Gould and Eugene Commins and disbanded in 2002. To make further progress, a proof-of-principle laser-cooled fountain EDM experiment was performed. The analysis of that experiment and an internal LBNL review identified signal intensity, systematics suppression, and magnetic noise as needing further improvement to reach an electron EDM limit of $2 \times 10^{-50} \mathrm{C}-\mathrm{m}$. Electron EDM experiments search for a difference in energy between an unpaired electron aligned and anti-aligned with an external electric field. High atomic number paramagnetic atoms such as Cs, Tl, and Fr provide test systems of zero net charge and, due to relativistic effects, enhanced sensitivity to an electron EDM.

The aim is to complete a linear optics solution for focusing Cs atoms that will result in most of the atoms passing through the electric field and being detected, to have performed calculations that show how to reach systematics limits well below $2 \times 10^{-50} \mathrm{C}-\mathrm{m}$, and to have constructed a 3layer prototype magnetic shield designed to reach a transverse shielding factor of $5 \times 10^{7}$.

Accomplishments:

A linear optics solution has been completed. Focusing is needed to compensate for the endfield defocusing of the electric field plates. This focusing is provided by an electrostatic triplet lens upstream of the electric field plates and at the same potential as the field plates. The shape of the triplet lens provides a combination dipole and sextupole field that acts as a transverse defocusing-focusing-defocusing lens combination, resulting in net focusing of the atoms in both transverse directions. End-field defocusing reduces transmission by a factor of 2000; the triplet lens prevents $95 \%$ of that loss. Several drafts have been prepared and a paper will be submitted for publication on this work.

A four-layer, $1 / 2$ scale prototype magnetic shield assembly has been constructed, along with magnetic coils for degaussing the shields and reducing the ambient field in all directions. In addition, coils have been constructed for perturbing the field to allow measurements of the shielding factor in longitudinal and transverse directions, as well as coils to allow feedback compensation for ambient field changes.

Calculations have been made that show a systematic error in the electron EDM of $2 \times 10^{-50} \mathrm{C}-\mathrm{m}$ is achievable. These calculations are based upon observables realizable with actual lasers, and take into account electron EDM mimicking parameters by using an appropriate combination of data taken with different angles for the analysis laser and different initial atomic states to cancel both mimicking parameters. In addition, the experiment uses electric-field quantization with no applied magnetic fields, and is designed to depend on the average electric and magnetic fields, minimizing sensitivity to local trajectory effects. A summary of this work has been sent out for review, and a draft for publication will be produced. 


\section{Structure Solution of Inorganic Materials using Energy Resolved Laue Microdiffraction}

Principal Investigators: Martin Kunz, Nobumichi Tamura (ALS)

\section{Project description}

Laue X-ray microdiffraction is a powerful tool for mapping grain orientation and strains in polycrystalline materials and single crystals with submicron spatial resolution. The use of white radiation allows simultaneously satisfying Bragg condition for a number of reflections. However, Laue diffraction is intrinsically "blind" to the energy attached to the individual reflection so that the use of their intensity is usually neglected. The purpose of this LDRD project is to develop software and experimental tools to enable routine structure solution and structure refinement from micron- and sub-micron grains as typically investigated in x-ray microdiffraction experiments. This will open ALS beamline 12.3.2 to an entire new set of scientific problems and thus increase its scientific impact.

\section{Accomplishments}

In the first year of this LDRD project we focused on the problem of interpreting observed intensities. The main obstacle here is the proper application of energy dependent correction factors to each individual reflection. Our main achievement was the development of a method to extract an effective flux curve for a given combination of Xray source and area detector. Knowledge of this parameter is pivotal for the successful interpretation of Laue patterns and non-trivial for cases where data redundancy is lacking (e.g. for small unit cell crystals of inorganic compounds). This method (dubbed "the reversed method") is based on comparing the calculated structure factors of a well characterized standard sample with its Laue intensities after applying a suite of analytical corrections (absorption, polarization, harmonic deconvolution). This method is also - to the best of our knowledge - the most direct way to measure the spectrum of a white X-ray source. The reverse method is the subject of a publication submitted to Journal of Applied Crystallography.

The effective flux determined by this way is transferable and allows to extract structure factors for any indexable phase. As a demonstration of the successful transferability we managed to assign the correct setting of a hexagonal unit cell to primitive trigonal lattice. This was previously not possible since it requires a proper assessment of the measured intensities. This opens the way to the determination of strain and stress in materials also with trigonal symmetry, and to check for the presence of twins in such material. A publication on this first application is in preparation.

The systematic exploitation of the reflection intensities opens new possibilities in the use of Laue X-ray microdiffraction. It will allow precisely monitoring rapid structural modifications as well as determining structure of unknown crystals in difficult cases not solvable by conventional monochromatic techniques (such as very small crystals, crystals embedded in a heterogeneous matrix, non stationary sample in suspension in a liquid or inside a diamond-anvil cell). This part will be demonstrated with zeolite samples in collaboration with Ch. Baerlocher and L. McCusker of ETH Zürich. 
The Nanoscale surveyor

Principle Investigators: Stefano Marchesini (Advanced Light Source)

Co-investigators: David Shapiro, Tony Warwick, Rich Celestre, Janos Kirz, (ALS) Frank Svec, (Molecular Foundry), Peter Denes (Engineering) Chao Yang, (CRD), Jacobsen (SUNY Stony Brook), J. C. H. Spence (ASU), (E. Kramer UCSB, RA. Segalman UCB)

This project aims to develop an x-ray instrument capable of terapixel high-throughput chemical mapping of nano-pourous materials at the resolution of 10 nanometers in three dimensions, and establish the requirements of one nanometer resolution target (detectors, light sources, algorithms, high performance computing). The combination of microscopy and diffractive techniques, referred to as ptychography, is only now made possible thanks to the convergence of three technologies: fast parallel x-ray detectors, robust phasing algorithms, and high-brightness x-ray sources. The capability to perform surveys, navigation over large areas as well as high resolution 3D chemical mapping will revolutionize the characterization of materials based on nanoparticles, which offer opportunities in energy applications ranging from solar cells, light-emitting devices, batteries, fuel cells, and hydrogen storage.

Accomplishments:

In the last fiscal year, we have focused on developing the experimental capability using a newly developed fast CCD detector developed at LBNL.

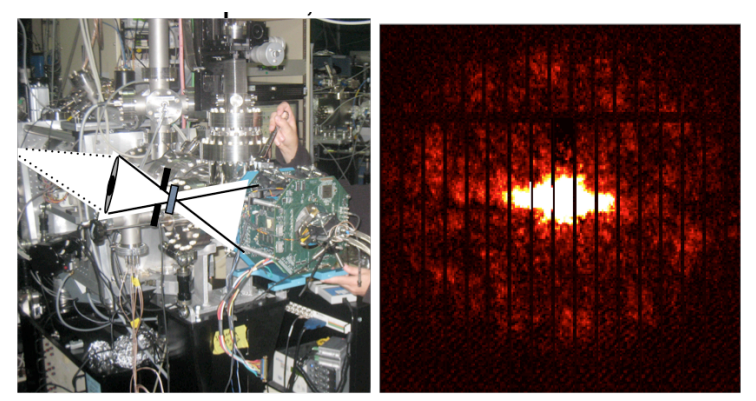

Fig 1. First experiments of this LDRD at the coherent imaging beamline at ALS (fig. 1), using a newly developed fast CCD detector (first diffraction frame on the right). and a focusing element indicate that that sub $10 \mathrm{~nm} 3 \mathrm{D}$ chemical mapping is possible within 1 year. Sustained data rate of $100 \mathrm{MB} / \mathrm{s}$ promise instant feedback on focal position, sample drifts and other experimental problems. Data is currently being processed off-line using custom code based on multicore SPMV/cuFFT libraries developed within this LDRD.

In addition to developing the orientation determination problem, we have also begun the investigate techniques for high throughput reconstruction and phase retrieval in which a number of diffraction patterns obtained from a set of overlapping subframes of the unknown object is used to uncover the object. We developed an optimization-based approach and analyzed the convergence of various algorithms for solving this type of problem. We have developed algorithms that enable blind recovery of the illumination function, position errors and increased tolerance to vibrations, and developed reconstruction software for multiscale reconstruction using multicore GPU libraries. We are in the process of writing up the results. 


\title{
High quantum yield multi-alkali cathodes for psec pulsed electron sources
}

\author{
PI: H. Padmore
}

\section{Project Description}

Many experiments require sources of psec dense electron beams, from those to be used for electron diffraction to free electron lasers. The generation of these beams is typically done using laser excitation on a metallic substrate. While robust, these cathodes are inefficient, typically having a quantum yield in the UV of 1e-4. The goal of this work therefore is to design and test new types of cathode based on high yield semiconductors. The system we have chosen to work with was discovered 40 years ago, but has only had limited success in pulsed electron sources due to issues related to dark current and fragility of the emitting surface. The main system we plan to work with is $\mathrm{K} 2 \mathrm{CsSb}$, which in the green part of the spectrum can have a QE of $10 \%$. Our goal is to understand the growth and surface chemistry of this system so that it can be reliably used in high current pulsed electron applications.

\section{Accomplishments}

We have now commissioned an MBE system dedicated to the production of high quantum yield semiconductor photocathodes. We have reacted $\mathrm{Sb}, \mathrm{Cs}$ and $\mathrm{K}$ to form a di-potassium cesium antimonide system with variable stoichiometry.
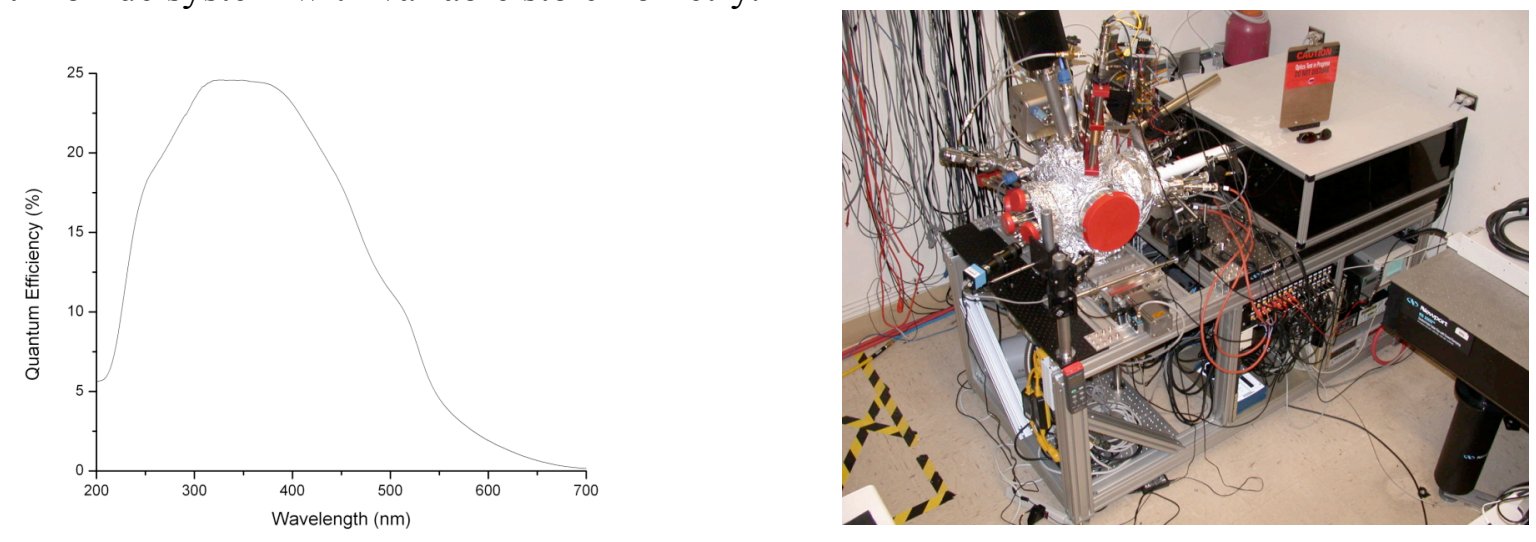

Fig. $1 \mathrm{QE}$ of K2CsSb (left) and overview of our alkali antimonide deposition system

We have now produced cathodes with up to 7\% QE in the green $(532 \mathrm{~nm})$, equivalent of $15 \%$ $\mathrm{QE}$ in the high field gradient of our RF accelerating gun. We have also demonstrated lifetimes of 1 day at 1e-9 of water, extrapolating to weeks of operation in the UHV of the gun. The fabrication process is complex and we are now concentrating on understanding the growth mechanism of the films using XRD and XPS. The next stage in the project will be to demonstrate reliable automatic production of cathodes, and their qualification under pulsed laser excitation conditions. The system will be used with microJ $532 \mathrm{~nm}$ green laser running at 1 $\mathrm{MHz}$. We are also in the process of installing a system for monitoring the stoichiometry of the materials as they are produced in UHV. 
An improved electron detection system for

"Extreme" angle-resolved photoemission experiments

Principal Investigators: Eli Rotenberg and Aaron Bostwick

\section{Project Description}

In this project we aim to implement a new lens for measuring the angular distribution of electrons for angle-resolved photoemission spectroscopy (ARPES) in "extreme" conditions such as ultralow temperature and nanoscale probe size. Such a lens is essentially an angular electron microscope because it collects angular, not spatial images. Existing commercial ARPES lenses work by selecting electrons emitted along a plane normal to a sample's surface. Acquiring data from other planes is necessary for successful ARPES measurements and is normally

accomplished by rotating the sample surface or rotating the detector, neither of which is possible in extreme environments such as in high pressures or with high spatial resolution requirements. The project aims to build an all-electronic scanning system which accomplishes this without requiring sample or detector motion, a capability which is not presently available anywhere in the world. Its development will not only support the user program at the nanoARPES endstation at the ALS (part of wave 1 of the ALS strategic plan) but can also be commercially viable and could be licensed should a patent be filed for it. We have previously developed a working unit with $12 \times 12^{\circ}$ scanning range and found no significant aberrations. Our goal is now to develop a new lens with $30 \times 30^{\circ}$ acceptance in order to sample a full unit cell in electron-momentum space.

\section{Accomplishments}

The present proposal is a continuation of an FY2009 LDRD. In the previous year we designed the lens through simulation. This year's LDRD project funded fabrication, assembly, and testing of the new lens system. Our main accomplishment was to successfully construct and test an angular scanning lens capable of nearly the desired design range. The figure below shows the results. The left two panels show band maps acquired in the standard dispersive direction and in the new deflector direction respectively. The right panel shows a 2D constant energy cut. All images were acquired without moving the sample.
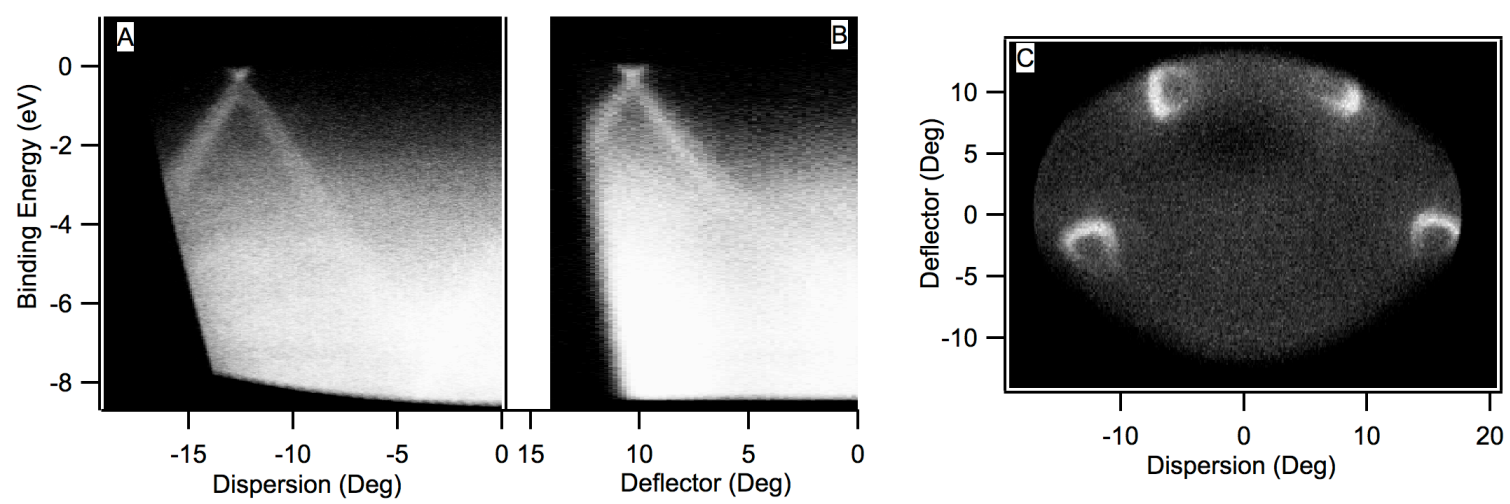

Figure. Test ARPES data acquired with the new lens on bilayer-graphene. A) Band map with standard dispersive capability. B) Band map using new scanning mode. C) Constant energy cut utilizing both dispersive and scanning capabilities. 


\title{
Ambient pressure photoemission spectromicroscopy
}

\author{
Principal Investigators: Hendrik Bluhm, Zahid Hussain, and Tolek \\ Tyliszczak
}

\section{Project Description}

The goal of this project is to design and construct a scanning ambient pressure photoemission microscope (AP-SPEM) with a spatial resolution of better than $100 \mathrm{~nm}$ for the investigation of heterogeneous chemical reactions at solid/vapor interfaces under conditions relevant to atmospheric/environmental science, energy research and heterogeneous catalysis. The investigation of surfaces under realistic conditions of temperature, gas composition and pressure is essential for a fundamental understanding of the molecular processes at liquid/vapor and solid/vapor interfaces that govern interfacial chemistry. Many of the most relevant solid/vapor interfaces are spatially and chemically inhomogeneous. In current ambient pressure photoemission spectroscopy (APPES) experiments this aspect cannot be adequately evaluated since the measurements average over the whole sample area that is illuminated by the incident $\mathrm{X}$-ray beam, which is usually $100 \mu \mathrm{m}$ in diameter or larger.

In the new instrument under construction we are combining the principle approaches of zone plate based scanning transmission X-ray microscopy (STXM) and APPES. We are implementing the AP-SPEM in the new APPES endstation at beamline 11.0.2. This endstation features a $3^{\text {rd }}$ generation differentially pumped electrostatic lens system with an acceptance half angle of better than $20 \mathrm{deg}$. Custom-designed sample cells can easily be attached and exchanged. The main challenges in the design and operation of a zone plate based APPES instrument are the due to the close proximity of zone plate and order sorting aperture (OSA) to the conical entrance aperture of the differential pumping system and the sample; the requirement for very precise alignment and motion of zone plate, OSA and sample; and the limit on the relative vibration of zone plate and sample to less than about $20 \mathrm{~nm}_{\mathrm{pp}}$

\section{Accomplishments}

In the first stage of the project we have developed the conceptual layout of the AP-SPEM. A custom-designed vacuum chamber was designed and is currently being machined. The type and specifications of the piezo motors and motor controllers for the scanning stages were determined and all parts procured. Position control by laser interferometry is essential for accurate spatial measurements; the laser interferometer that controls the positioning has also been specified and purchased. Initial test measurements on the performance of the scanning stages have been carried out. In addition, the sample manipulator was specified and purchased. The new electron energy analyzer with differentially pumped lenses, to be used as the main detection system, has been commissioned. Recently, Andrey Shavorskiy has joined the project as a postdoctoral research fellow. 
Test M onochromator/Spectrometer Systems with Prototype High Density Gratings for High Resolution $X$-ray Scattering

Principal Investigator: Tony W arwick

\section{Project Description}

R esonant Inelastic soft X-ray Scattering (RIXS) measures the energy scales of soft excitations which are normally obscured by lifetime broadening. The main technical issue is to achieve the high energy resolution needed ( $1 \mathrm{meV}-10 \mathrm{meV})$ at the photon energies required to access relevant core levels (500 eV - $1.3 \mathrm{keV}$ ). The goal of this work is to design, build and operate small, novel, test monochromator-spectrometer systems to precisely evaluate the $\mathrm{x}$-ray dispersive quality of prototype high-density multilayer diffraction gratings. These systems will transform the instrumentation for ultrahigh resolution RIX S from extremely large spectrometers to smaller instruments, able to perform at even higher spectral resolution within the space of the A LS floor.

A simple high resolution multilayer-grating test monochromator will be designed and constructed to measure the efficiency and the precise dispersive and optical properties of prototype high resolution multilayer gratings.

M ultilayer (M L) coated blazed gratings will be developed with a high groove density operating in a high diffraction order. A chemical etch of assymetrically cut silicon substrates will be developed to produce the smooth facets on which multilayer deposition can be made to produce gratings with high efficiency. Prototype gratings constructed by various techniques will be measured. Numerical simulations of grating errors will be performed.

\section{Accomplishments}

W e developed a process for fabrication of high quality EUV diffraction gratings with an effective groove density of 15,000 lines $/ \mathrm{mm}$. Substrate blazed gratings were made by scanning beam interference lithography and anisotropic $\mathrm{KOH}$ etching of silicon. The optimized anisotropic etching provides excellent control of the slope of blazed facets, high smoothness of the facet surface, and very short anti-blazed facets. A new nub removal step provides a triangular substrate groove profile which is close to the ideal one.

The diffraction efficiency of the multilayer coated blazed grating was measured at the A dvanced Light Source beamline 6.3.2 two-axis diffractometer. W e report measurements that show, for a $5000 \mathrm{l} / \mathrm{mm}$ grating diffracting in the $3^{\text {rd }}$ order, a diffraction efficiency of $37.6 \%$ at a wavel ength of $13.6 \mathrm{~nm}$, close to the theoretical maximum. 
X-Ray Optical Metrology for Coherence-Preserving Adaptive Optics Principal Investigators: Kenneth A. Goldberg, Valeriy Y. Yashchuk

\section{Project Description}

The goal of this LDRD project is to develop the methodology of x-ray nanofocusing by integrating ex-situ optical testing and in-situ x-ray wavefront sensing on high quality x-ray optics. Some of the most exciting frontiers of materials science occur at the nanoscale, where self-organized nanostructures, correlated materials, micro-crystallites, and engineered nanostructures exhibit complex behaviors that deepen our understanding of basic properties of matter. These areas of nanoscience are driven forward by the development of new probes and techniques with nanometer-scale resolution. Among them, techniques in synchrotron radiation play a central role due to match between the nanoscale and soft x-ray wavelengths, and the availability of focusable short-wavelength light generated by extremely high brightness third or fourth generation synchrotron light sources, such as the ALS. However, the important advantages of synchrotron light sources are limited by the quality of available beamline (BL) optics: optics performing at the state of the art suffer from insufficient figure and finish quality, shape misadjustment, thermal drifts, and in situ misalignment.

We propose to develop high-precision in situ surface metrology capable of providing effective feedback for future adaptive x-ray optical components. Among the techniques to be developed and tested are Hartmann wavefront sensing, shearing interferometry, and emerging interferometric and non-interferometric methods. These methods have recently been used in the creation of diffraction-limited EUV optical imaging systems with sub-nanometer tolerances, and in prototype BL alignment systems elsewhere. However, so far, the techniques that have been developed for BL optics are limited to passive metrology, and have not demonstrated sufficient sensitivity for nanofocusing. We propose further development of these techniques to provide metrology with higher sensitivity and efficient shape-control feedback. Our goal is to deliver methods that can be replicated and incorporated into various BL designs, and which set the stage for automated mirror shape control.

\section{Accomplishments}

In FY2010 at ALS beamline 5.3.1, we have been developing experimental techniques to surpass sub-micron focusing with Kirkpatrick-Baez (KB) mirrors. We developed an original technique to precisely pre-align the vertical displacement and tilt angle of a Kirkpatrick-Baez (KB) mirror. Fine alignment was conducted with a scanningslit technique that uses the method of characteristic functions and linear regression, adapted from the ex situ mirror-bending methodology. We also developed an experimental set-up to enable soft x-ray metrologies, including shearing interferometry, and quantitative knife-edge testing. Using these in situ metrology techniques we performed the first interferometric measurements of the surface profile of a temperature stabilized KB mirror, and we optimized the mirror alignment using soft x-ray light. The preliminary comparison of the results of the in situ and ex situ metrologies demonstrated 1-nm-wavelength focusing down to 120-nm FWHM, close to "diffraction-limited" performance. We believe this work seeds the creation of larger scale DOE metrology programs with LBNL in the leadership role. 


\section{Chemical Sciences Division}

Direct Comb Spectroscopy of Lithium in the Vacuum Ultraviolet and Beyond

Principal Investigator(s): Dmitry Budker and Holger Müller

Project description:

The purpose of this project is to develop technologies required to generate coherent femtosecond ultraviolet (uv) light, as a basis for a later extension to soft x-rays. Furthermore, it is to study the application of such light in atomic and molecular physics, by performing direct-comb spectroscopy of cold lithium atoms and using laser combs for atom optics. In order to achieve these goals, we will initially work in the uv spectral region, but apply the technologies and methods that are envisioned for experiments in the soft x-ray region. This will allow us to gain the necessary experience by studying the limitations of these methods at low photon energies, where experiments are much simpler and, thus, provide the flexibility required to find the optimum experimental strategy.

Our strategy is to trap Lithium from background vapor in a 2 dimensional magnetooptical trap (2D-MOT), transfer it into a 3D-MOT, and then store it in an optical dipole trap. A femtosecond (fs) oscillator will be stabilized to a high-finesse cavity and harmonics will be generated within a dispersion-compensated enhancement cavity. Methods for intracavity phase matching will be studied. Direct comb spectroscopy of laser-cooled lithium will be performed using single-photon and two-photon sum frequency excitation. This work will serve as a test-bed and proof of principle to get us ready for eventually creating frequency comb metrology in the $\mathrm{x}$ ray spectrum. Another important goal is to investigate the pressure broadening of the $\mathrm{CO} 2$ absorption bands is useful for gathering data necessary for monitoring atmospheric $\mathrm{CO} 2$.

Accomplishments:

We have acquired, installed, and tested the frequency comb, a Menlo Systems FC8004, based on a mode-locked femtosecond titanium:sapphire laser pumped by a low noise Coherent Verdi V-

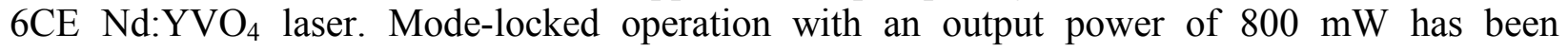
achieved. We have broadening the comb to an octave-spanning spectrum using a microstructured optical fiber; $150 \mathrm{~mW}$ of octave-spanning spectrum are generated. Furthermore, carrier-envelope phase stabilization of the comb at a repetition rate of $200 \mathrm{MHz}$ has been achieved. A frequency reference for it is being constructed using a high finesse (nominally, $F=100,000$ ) Fabry-Perot cavity. The cavity is a rugged monolithic design, based on a $5 \times 5 \mathrm{~cm}$ cylinder of ultralowabsorption quartz glass (Suprasil 3001) coated with high reflectivity dielectric stacks.

For the lithium trap, we have built the master laser for the magneto-optical trap (MOT), an ECDL that uses a Hitachi HL6555G Fabry-Perot laser diode and a holographic grating having 2400 lines $/ \mathrm{mm}$. It delivers $15 \mathrm{~mW}$ of single-frequency output power. Mode-hop free operation of the laser has been achieved; the laser has been frequency stabilized ("locked") to any of the D2 lines in ${ }^{6} \mathrm{Li}$ or ${ }^{7} \mathrm{Li}$ using Doppler-free modulation transfer spectroscopy in a heat pipe vapor cell, in which a Li sample is heated to $355 \mathrm{C}$ to achieve sufficient vapor pressure. We have sealed off the heat pipe and operated it for 8 weeks without pumping. We have locked the laser by applying feedback to the laser diode injection current and the grating. 


\section{Probing Transient Molecular Entanglement using \\ Femtosecond High Resolution Delayed-Field Coincidence Imaging \\ Principle Investigator(s): Oliver Gessner}

\section{Project Description}

This project is aimed to study the transient entanglement of the constituents of a molecule during unimolecular dissociation in real-time. It will explore the very heart of chemical bonding: the boundaries and the transition between separate particle and unified molecular behavior. The quantum mechanical nature of chemical bonding over potentially macroscopic distances and the conditions to create entangled particles through unimolecular dissociation in the gas phase will be investigated.

A newly designed high-resolution delayed-field photoelectron-ion coincidence imaging spectrometer will be used in combination with the high repetition rate ultrafast soft X-ray source of the Ultrafast X-ray Science Laboratory (UXSL) at LBNL. The studies will constitute a significant contribution to the UXSL endeavor in becoming one of the world's leading centers for ultrafast X-ray science. They will aid in paving the way for future science performed at ultrafast X-ray light sources like the Stanford LCLS, and help to define benchmark requirements for a new FEL-based ultrafast X-ray source at LBNL.

Accomplishments

A series of femtosecond time-resolved photoionization studies has been performed using both a momentum imaging ion detector as well as a Velocity Map Imaging (VMI) photoelectron spectrometer. The combination of this spectrometer configuration with a state-of-the-art femtosecond EUV laser light source provides an absolute cutting edge tool to explore ultrafast chemical dynamics in electronically excited molecules.

Experiments on helium nanodroplets have demonstrated the power of the novel ion detector technique to record mass-, angle-, and kinetic energy-resolved ion spectra in a non-dispersive fashion. The combination of photoelectron and ion imaging data proves to be instrumental to disentangle various relaxation pathways after electronic excitation of the superfluid clusters. In a next stage, both spectrometers will be operated in coincidence, which will provide the full power of kinematically complete photoionization experiments.

First experiments on hexafluorobenzene have demonstrated the capability of the setup to record femtosecond time-resolved quantum coherence phenomena in polyatomic molecules. The analysis of the data is ongoing.

A U.S. non-provisional patent application has been filed by the LBNL patent group for an ultracompact VUV spectrometer and beam-profiler that has been developed in the course of this LDRD project. The technology is currently marketed for licensing by the LBNL technology transfer group. 
Development of in situ cells for Reactive Spectroscopic and Microscopic Studies Principal Investigator(s): Mary Gilles

\section{Project Description}

This proposal is to develop in situ cells for scanning transmission x-ray microscopy (STXM) studies of nanoscale systems relevant to energy production. Potential applications include: biomass energy production; syngas production; and the development/improvement of solar cells. In situ cells are critical components for advancing studies in a wide range of fields; atmospheric chemistry, solar cells; electrochemistry (i.e. batteries), soil chemistry, magnetic systems, polymer processing, and catalysis. Commonalities between all of these include the need to understand detailed changes in chemical composition on the scale of 10's of $\mathrm{nm}$ to microns in reactive environments.

The goal is development of several in situ cells that are robust and can be adapted to other experimental platforms at the Advanced Light Source, including Fourier transform infrared spectroscopy, X-ray fluorescence microprobe techniques, or bulk near edge x-ray absorption fine structure (NEXAFS) measurements and micro-Raman. Since laboratory based ultrafast (femto or attosecond) transient absorption experiments share similar sample requirements with STXM, these cells could be readily adapted to laboratory based experiments.

Essentially three types of cells will be developed: a micro fluidic cell, a temperature controlled reactor (reactive gas flow and varying relative humidity); candidates for the third cell include either a micro fluidic fuel cell or an electrochemical cell.

\section{Accomplishments}

Two iterations of the gas flow cell were developed. The first was a two piece ceramic based system which was proved to be insufficiently robust for application. The improved cell is manufactured from aluminum and incorporates the mounting bracket for precise placement into the scanning transmission X-ray microscope as well as incorporated the flow tubes securely into the cell.

Two versions of the silicon nitride membranes with the heating spiral were manufactured. One with a gold based heater, the second for higher temperatures incorporates Pt. These were just recently received and the next step is determination of the temperature gradient across the membrane. As the temperature gradient across the silicon nitride membrane is expected to be large, chemical methods to characterize this gradient with a few hundred nanometer spatial resolution were designed. The equipment and chemicals necessary for these measurements recently arrived.

Static conditions were demonstrated for the fluidic cell with an absorption pathlength on the order of $200 \mathrm{~nm}$. Conditioning the silicon nitride windows and incorporating syringe pumps, we were also able to demonstrate flow in this system. A second system has been manufactured with a design that allows it to be placed into the scanning transmission X-ray microscope. This is ready for preliminary testing prior to insertion into the microscope where the ability to monitor and actively control the pathlength will be characterized 
Dynamics of homogeneous catalysis reactions investigated with transient twodimensional infrared spectroscopy

Principle Investigator: Charles B. Harris

\section{Project Description}

Organometallic compounds have broad applications in synthetic chemistry, particularly as homogeneous catalysts. A full understanding of the mechanisms and associated dynamics of organometallic reactions allows new synthetic routes to be designed and current syntheses to be improved. Direct experimental observation of these dynamics is often the best way to determine the details of these transformations. We have previously studied organometallic catalytic systems using transient one dimensional infrared spectroscopy, in which a reaction is photoinitiated by a UV pulse and then probed by an IR pulse. We are currently expanding our study of photochemical reactions using transient 2D-IR (T2D-IR). Thorough analysis of the T2D-IR spectra can give information on the structure of not only the transient intermediates but also the transition states connecting them-details that conventional spectroscopic techniques cannot provide. In order to show the efficacy of this technique, we are performing a proof of principle experiment in which we are studying the photolysis of iron pentacarbonyl to iron tetracarbonyl. We then intend to use our expanded multidimensional capabilities to study important homogeneous catalytic reactions such as selective $\mathrm{C}-\mathrm{H}$ bond activation by a novel boryl complex, $\mathrm{Cp} * \mathrm{~W}(\mathrm{CO})_{3}(\mathrm{Bpin})$ (pin $\left.=1,2-\mathrm{O}_{2} \mathrm{C}_{2}-\left(\mathrm{CH}_{3}\right)_{4}\right)$ in neat pentane solution (Sawyer et al., J.Am.Chem.Soc. 2010, 132, 1848-1859).

\section{Accomplishments}

In the past year, we have nearly completed our theoretical treatment of the photolysis of iron pentacarbonyl. To fully interpret T2D-IR spectra, it is necessary to have density functional theory (DFT) calculations describing the geometry and vibrations of all species involved in the reaction. By projecting the vibrational displacement vectors from the reactants to the transition state and product vibrations, we predict how vibrational energy will couple from reactant to product in the cases of axial or equatorial CO loss. Both geometry and vibrational projection calculations have been completed, allowing us to compare the predicted spectrum associated with a particular mechanistic pathway (Fig. 1).

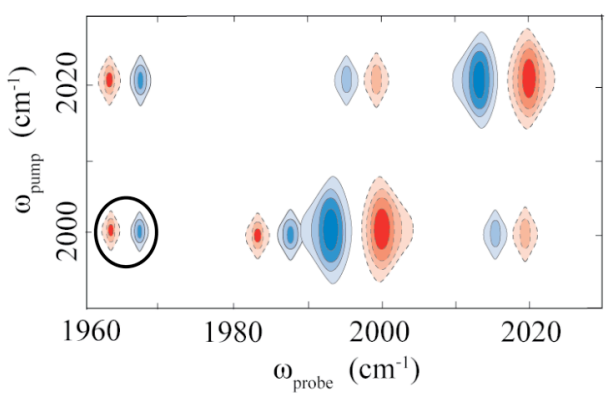

predicted ax. CO loss

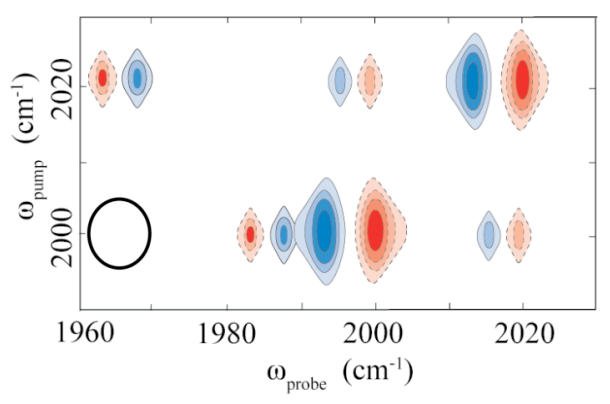

predicted eq. CO loss
Fig. 1: Predicted spectra for $\mathrm{Fe}(\mathrm{CO})_{5} / \mathrm{Fe}(\mathrm{CO})_{4}$ photolysis reaction based upon DFT calculations.

Note that the product peak at $1965 \times 2000 \mathrm{~cm}^{-1}$ (circled) will not appear if the mechanism is primarily equatorial $\mathrm{CO}$ loss.

Further spectroscopic data has also been taken in our study of $\mathrm{Fe}(\mathrm{CO})_{5}$ photolysis. These spectra indicated that the quantum yield of the photoreaction was not high enough to study the reaction dynamics of vibrationally labeled molecules with the laser system in that configuration. We have spent the previous several months reconfiguring the way in which we split the energy of our pulses between our UV and IR lines, as well as a second, recently-constructed mid-IR OPA that should allow us to achieve up to $70 \%$ more UV pump intensity. When these upgrades to the experimental apparatus are completed, T2D-IR data will be forthcoming. 
Investigating f-Electron Exchange Coupling in Actinide and Lanthanide Complexes

Principal Investigator: Wayne Lukens

\section{Project Description}

The purpose of this project is to better understand exchange coupling in actinide and lanthanide (f-metal) complexes. Specifically, the goal is to develop techniques to quantify exchange coupling in these systems; currently, no general approach exists that allows quantification of exchange coupling involving f-electrons. This information will allow a better understanding of the roles that f-electrons play in bonding and determining magnetic behavior, especially as it relates to lanthanide-based single molecule magnets and to "Kondo-like" complexes of certain lanthanides, particularly ytterbium and cerium.

We will develop new techniques for quantifying exchange coupling in these systems, which use the crystal field information contained in the magnetic susceptibility of structurally similar complexes that do not display exchange coupling. In this approach, it is not necessary to determine the crystal field parameters, which is extremely difficult for low-symmetry systems. Instead, the magnetic susceptibility itself will be used to quantify the exchange coupling in other systems. We will prepare a series of lanthanide and actinide complexes to test and expand these techniques. If possible, the data obtained will be also be analyzed by traditional method, which does involve determining the crystal field parameters, which are then used to diagonalize the full Hamiltonian for the coupled system. In this way, the new, general approach can be compared to a more rigorous approach.

\section{Accomplishments}

The new approach has been applied to systems involving two, identical f-metals. In all cases, the exchange coupling can be quantified by modeling the magnetic susceptibility of the coupled system using the new approach. However, for these systems, it is not possible to compare the new approach with the more rigorous approach because the existing data are insufficient to calculate the crystal field parameters.

To address this problem, a series of lanthanide complexes in which the lanthanide center is coupled to an organic radical has been prepared along with a series of related complexes that do not possess an organic radical but are otherwise similar. The magnetic susceptibility of these complexes has been measured. The magnetic susceptibility of the complexes without organic radicals should be sufficient to determine crystal field parameters.

We are in the process of determining the crystal field parameters for these complexes. In addition, we are in the process of refining the approach originally used to quantify exchange coupling between two identical f-ions to allow quantification of exchange coupling between an $\mathrm{f}$ ion and an organic radical. 


\section{Double-Auger Emission of small Molecules following Core-Excitation \& Ionization}

Principal Investigator(s): Thorsten Weber

\section{Project Description}

The goal of this project is to develop a 5-particle detection and analysis scheme and to prove its functionality in an ambitious research program, which focuses on the double Auger decay of small CO molecules after photo excitation and photo ionization of inner shell electrons. Here the aim is to understand the dissociation pathways during the Auger decay and the photo-electron valence-electron correlation; a fundamental correlation yet unexplored, which plays an important role in the understanding of the shape resonance energy-bond-length correlation in chemical compounds. Moreover, we want to investigate post collision interaction effects in the emission patterns of the outgoing electrons, and thus hunt for a breakdown of the widely accepted 2-step mechanism in small molecules.

As mentioned above, these complicated Atomic, Molecular, and Optical (AMO) investigations will require coincident 3d-momentum measurements of up to 5 particles, a yet unprecedented experimental undertaking. These challenging experiments will lay the ground work for future many particle measurements with intense (x-ray) free electron laser sources and high harmonic generation laser systems and act as a proof of principle. Especially at free electron lasers the intensity of the incoming light will be that high that 2 photons can simultaneously couple to 2 core electrons, resulting in two vacancies, which initiate rich correlated multielectron quantum dynamics and the emission of photo and Auger electrons to be measured time and position resolved. Currently we are setting up a so called Momentum Imaging Spectroscopy for TimE Resolved Studies (MISTERS) apparatus, which will function as a reaction microscope dedicated to experiments with ultrashort laser pulses. The project proposed here is directly linked to the completion of MISTERS.

\section{Accomplishments}

We finished the simulation and design phase of our spectrometer needed to make the coincident 3d-momentum imaging experiments of up to 5 particles ( 2 ions +3 electrons) possible. Different sets of electric and magnetic fields have been chosen and an electrostatic lens for the position focusing on the ion side has been employed. We succeeded to implement two time and position sensitive detectors with so called hexagonal anode readout. We found out that on the electron side the use of that type of detector is indispensable for multihit reasons. On the ion side this scheme helps us to improve the position resolution while fully exploiting the capabilities of the electrostatic lens. This is the first time that two of such complex detectors are used simultaneously and this setup prepares us for even more demanding molecular break-ups in the future.

Since the granted funds were capped the desired transient recorders (flash ADCs) for an efficient electronic pulse processing could not be purchased. We changed plans accordingly and we worked on an alternative and improved TDC based solution which allows us to read out the timing signals of the multihit event. A first (test)experiment on Ne Atoms was performed during the two bunch mode of the Advanced Light Source Synchrotron (ALS) ring in August 2010. The crucial home made fast electronics were successfully set up and optimized also. An improved version of this setup will be employed in the next experiment on CO molecules which is scheduled for March 2011 at the ALS. A very complicated analysis of the 15d-phase space will follow afterwards, which is aimed at sorting the data and singling out the correlated multihit events. After this we plan to visualize the results in highly differential spectra which will enable us to answer the scientific questions mentioned above. 


\section{Computing Sciences}

Enabling HPC Workflows on Clouds

Principal Investigator(s): Deborah Agarwal and Keith Jackson

Project Description

The promise of on-demand large-scale 'personalized' computing capacity has attracted many DOE science applications to the commercial cloud offerings. Utility, or "cloud", computing is quickly becoming the dominant paradigm for delivery of costeffective, scalable, energy-efficient computing resources. Clouds promise to deliver the ability to submit an application from a desktop and take advantage of personalized ondemand resources all over the world.

Cloud computing is still early in its development and a wide array of cloud computing paradigms have emerged. As clouds become more ubiquitous, it is essential that DOE scientific applications be able to take full advantage of them. In this project we are working with a representative set of science applications to identify what is needed to adapt existing application software to the cloud and identify new software needed to enable science applications to readily utilize cloud resources. Through this work, we hope to identify common abstractions and techniques that enable effective use of the cloud infrastructure.

\section{Accomplishments}

Our most significant accomplishment has been to port the Nearby Supernova Factory (SNFactory) analysis pipeline into the Amazon Web Services Cloud, and conduct a detailed analysis of its cost and performance. The SNFactory pipeline involves running a complex series of image processing algorithms to search for transients. Currently the input dataset is about $10 \mathrm{~TB}$, and the output is on the order of 20TB. This pipeline must work for a decade or more. The Cloud is a very appealing environment for this application; through the use of virtualization, the environment (operating system, compiler, networking, etc) can remain stable to avoid costly rewrite requirements due to upgrades during the project's long lifetime. Because this application was originally written to run on a standard Linux cluster, significant effort was required to port it into a Cloud environment. Understanding how to effectively utilize the different abstractions provided in commercial Cloud infrastructures for scientific applications is an important part of this research. The result of this work is two papers: Seeking Supernovae in the Clouds: A Performance Study - that won the Best Paper Award at the 1st Workshop on Scientific Cloud Computing held in conjunction with HPDC 2010 and Performance and Cost Analysis of The Supernova Factory on The Amazon AWS Cloud - currently in submission to the Wiley Journal of Scientific Programming.

We have also begun to port our MODIS satellite imagery processing pipeline to run on the DOE Magellan Cloud testbed at NERSC. The processing pipeline consists of spatial and temporal sub-setting or aggregation, resolution transformation, and reprojection from one map projection to another. The current pipeline runs in the Windows Azure Cloud platform. By porting the pipeline into the Magellan Cloud environment, we expect to gain insight into the relative performance and programmability of disparate Cloud infrastructures, and begin to develop the necessary abstractions to support the effective utilization of Cloud infrastructures for data-intensive scientific applications. 
Low Order Models for Simulation of Ballistic Charge Transport in Nanoscale Devices

Principal Investigator: John B. Bell

\section{Project Description}

As size of electronic devices shrinks to nanometer scale, ballistic charge transport becomes increasingly important in describing the transport phenomena in these devices. However, its simulation is usually computationally intensive due to the large number of states required to accurately determine the distribution of the electron density and the number of self-consistent iterations needed to achieve convergence. The project will develop a computational strategy that utilizes model-order reduction techniques. The goal is to validate and demonstrate the effectiveness of the resulting approach.

Our formulation for the ballistic charge transport is based on the Schröedinger Poisson system of equations that must be solved self-consistently. Our proposed approach integrates the reduced basis method, a model-order reduction technique which exploits dimension reduction afforded by the smooth and low-dimensional parametrically induced solution manifold, with the subband decomposition method. We also exploit $a$ posteriori error estimation procedure to systematically control the accuracy of our solutions. We have successfully applied the method to a 2D double-gate nano-MOSFET device. To extend the approach to 3D structures, we will also need to determine a more efficient solution method for solving the equations.

\section{Accomplishments}

In collaboration with a scientist at Massachusetts Institute of Technology, we explored the applicability of the Newton-Raphson method, which theoretically has better convergence properties. Due to the large number of eigenvectors we need to determine, a naïve implementation of the method will require solving a large system of equations for each Newton iteration. Our new implementation reduces the computational cost to that comparable to the fixed-point method. Further work is however needed to exploit the smoothness in the electron density, a property lacking in eigenvectors corresponding to electrons with higher kinetic energies.

We have also explored the applicability of model reduction techniques in multiscale flow simulations in porous media. We devised an approach to represent a correlated random permeability distribution on a simulation domain as union of independently generated distributions on smaller domains based on Karhunen-Loeve decomposition that preserves the statistical properties of the original distribution. The pressure equation can then be solved efficiently on each subdomain based on reducedbasis method, similar to the approach developed so far for the charge transport simulation. We are currently looking at application of this technique to areas in uncertainty quantification for subsurface flow. 
Enhancing the Effectiveness of Manycore Chip Technologies for High-End Computing Principal Investigators: Jonathan Carter, Leonid Oliker, and John Shalf

Project Description

The goal of this project is to investigate the key challenge for next-generation highend computing systems - effectively utilizing computing systems with exponentially increasing numbers of cores per chip. Current petascale computing platforms are constructed from chips containing 10's of cores, and that in the future exascale architectures will be comprised of chips with substantially more cores.

Our work this year has examined the utility of a broad range of manycore systems, exploring both heterogeneous and homogeneous designs that are projected to serve as building blocks in large computing platforms for scientific simulations. Since power consumption is becoming almost the primary issue with deploying high-end computers, power measurements for these technologies has also been considered as a key metric.

Accomplishments

This year we have focused our efforts on studying the Gyrokinetic Toroidal Code (GTC), a 3D Particle-in-Cell (PIC) application originally developed at the Princeton Plasma Physics Laboratory to study plasma micro-turbulence in magnetic confinement fusion. Turbulence is believed to be the main mechanism by which energy and particles are transported away from the hot plasma core in toroidal fusion devices called tokamaks. An in-depth understanding of this process is of utmost importance for the design of future experiments and possible commercial reactors, since their performance and operation costs are directly linked to energy losses.

Unlike many other applications, GTC's reliance on scatter/gather operations makes it notoriously difficult to optimize even in the sequential realm. In the shared memory environment of modern multicore chips, the resolution of fine-grained (word-level) concurrent access to shared data places GTC at the forefront of the programming challenges for the next decade. Although GTC has been used to simulate large experiments and run at extremely high concurrencies, it has been done so mostly via a weakly-scaling message passing approach. We have adapted GTC's principal kernels to a shared memory multicore environment to enable continued scaling, benchmarking AMD and Intel's latest quad-socket shared-memory multiprocessors (SMPs), including a 24core Opteron (Istanbul) and 64-thread Xeon (Nehalem-EX), as well as a Sun Niagara2 and a Tesla C2050 (Fermi) GPU. Moreover, we analyzed the relative advantage of this set of machines to their predecessors (Barcelona, Nehalem-EP, and GT200).

Overall our work presents numerous node-level methodologies to significantly improve performance of simulations, while providing in-depth analysis into the tradeoffs of emerging multi- and manycore designs for challenging classes of numerical computations. Our work points to the limitations of the manycore philosophy of simply instantiating as many cores as technology allows. 


\title{
Energy Smart Disk-Based Mass Storage System
}

\author{
Principal Investigator(s): Doron Rotem, Ekow Otoo
}

\section{Project Description}

The Energy Smart Disk-Based Mass Storage System project is intended to address system software approach for implementing energy efficient storage systems. The primary goal is to investigate, implement and deploy modules for the management of reliable low-cost disk-based storage systems that maximize the energy savings while meeting the performance goals of the storage systems with respect to access latency, reliability and operational costs. The main ideas involve: (i) making smart decisions when to power down idle disks based on analysis of historical workloads (ii) utilizing replication of data to save energy by scheduling i/o to replicas on spinning disks rather than unnecessarily spinning up disks iii) implementing energy aware cache replacement policies and algorithms for emerging hybrid-disks technology, i.e., disks augmented with flash memory. The project's goals also include the development of analytical and experimental simulation models for analyzing energy consumption of different configurations of these system and configuring a prototype system that embeds our solutions.

\section{Accomplishments}

During this year we investigated strategies that take advantage of data replication for energy savings. Data replication is commonly used in many data centers for the purpose of data recovery and fault tolerance. To this end we developed an algorithm called FREP (Fractional Replication for Energy Proportionality). FREP replicates data on disks such that the number of active disks can be adjusted based on observed workloads and service level agreements. The work on FREP (submitted for publication) includes a prediction model based on past historical observations to enable probabilistic decisions for optimal decisions for shutting down inactive disks. We provide extensive evaluation results with a diverse set of traces, including real life and synthetic traces with different workload characteristics.

In another research work, we proposed a novel energy-aware scheduling approach to reduce energy consumption of storage systems without interfering with data placement or introducing additional performance and deployment overhead. The contributions of that work include the following:

-We formulated energy-aware scheduling as an optimization problem and proposed both optimal and heuristic algorithms for offline, batch and online cases.

-We analyzed the complexity of the energy-aware scheduling problem and showed that several variations of it are NP-complete using reductions from the weighted set cover problem in the batch case and the maximum independent set problem in the offline case. In both cases we solve the scheduling problem sub-optimally using efficient polynomial approximations.

-We provided extensive evaluations with realistic traces, disk models and power managers. The experiments showed a reduction in energy consumption up to $55 \%$, while minimizing the number of disk spin-up/down operations and request response time. 


\section{From Images to Models to Computational Input}

Principal Investigator: J.A. Sethian

Project Description:

Our work concerns developing physics-based models to understand cell properties and mechanics. Our goal is to automatically extract quantitative information from images leading to tessellated triangulated models: these models serve as input to PDE-based mechanical force algorithms. To this end, we need to build several types of algorithms, including methods to extract geometric data from cell images, as well as highly accurate computational techniques to model the hydrodynamic, mechanical, and elastic/continuum forces involved in cell development. These two goals are interrelated: the extracted geometric data from cell images will serve as both an initializer and as checkpoints for the evolving computational model, and the full physics solver will be used to guide the gathering of experimental data.

The tasks and long-range goals are as follows: (i) Automatically characterize and cluster cells and tissues according to underlying properties, including geometry, shape, and texture; (ii)Automatically extract triangulated, tessellated models of tissue structure and architecture from high resolution imaging; (iii) Build algorithms which process these structural models and analyze shape properties such as cluster shapes (for example, round, mass, stellate, and grape-like), degree of orderedness, spheroidicity, etc.; (iv) Register time-sliced data across multiple imaging modalities, and use this data as input to PDE-based variational geometric equations of motion. In these equations, driving forces lead to balance laws, stable or unstable configurations and topological change. The goal is to use the triangulated data to serve as checkpoints in an attempt to reverse engineer the physical forces that lead to abrupt changes and instabilities; and (v) Use these models as initial data to a prototype hydrodynamic/elastic PDE-solver to test physics-based equations of motion for tissue stability and response to forces.

Accomplishments:

We have made progress on these goals.

- In collaboration with LBNL, UC Berkeley, and UCSF, we have been gathering image data of mammary epithelial cells, acquired using laser-scanning confocal microscopy. We have developed some prototype image processing algorithms to despeckle image slices, isolate and extract boundaries and membranes.

- We have explored PDE-based techniques to track the evolution of multiple regions. Rather than use a large number of tightly connected individual functions working in tandem, we instead use a different methodology for these multiple regions with highly complex shared membranes. Our approach evolves an initial value discrete-continuum PDE, defined on a computational mesh, which does not scale with the number of different regions. The discrete-continuum PDE avoids embedding and correctly satisfies singular motions at the interface junctions.

- Using the above algorithms, we have built a coupled PDE-interface projection method solver for interfaces moving under surface tension, incompressible fluid flow, geometry constraints, and including gaseous permeability effects. Here, we use a scheme to enforce fluid compressibility, our interface technique, schemes for the momentum equation, and where required, a fixed wall boundary condition for anchoring.

Our next goals are to couple these algorithms to the time-varying cell boundaries extracted from the image data. 


\title{
A Holistic Approach to Energy Efficient Computing Architecture
}

\section{Project Description}

\author{
J. Shalf, L. Oliker, M. Wehner
}

The goal of this project is to develop new technology for energy-efficient computational science. The approach is to take a vertical slice through the space of applications, algorithms, software, and hardware to perform a study on how to build the most energy-efficient system to solve a particular computation science problem. The initial target will be climate simulation, and the solution is likely to require higher degrees of parallelism in the climate algorithms and software, as well as new hardware designs based on technology from outside the traditional workstation and server domains, particularly low-power embedded technology. The result will be a proof-of-concept machine design, along with supporting analyses of how this compares to alternative designs, and new algorithms and software techniques as needed to make the case for feasibility of computing the science problem on the system. The project is led by John Shalf and will coordinate with other energy-efficient projects, including the LDRDs by Erich Strohmaier, Jonathan Carter, and Doron Rotem.

\section{Major Milestones in 2010}

This year marked a shift in focus from analyzing the climate code to demonstrating the delivered performance and energy efficiency benefits and direct comparisons with GPU and CPU architectures. The Green Flash architecture and holistic development approach has been re-purposed to support the DOE/ASCR initiative to develop energy-efficient exascale computing technology by 2018.

\section{Code Analysis}

We have expanded our collaboration with the Fraunhofer Institute (ITWM) to understand how our approach could be applied to energy-efficient computation for seismic imaging and geophysics applications. During the course of 2010, we analyzed Reverse Time Migration (RTM) code used for seismic imaging, and developed a version of Green Flash (called Green Wave) that was tailored for that algorithm. We also partnered with NVidia to develop a highly optimized version of that code for GPUs and worked with Sam Williams in FTG to use auto-tuning technology to tune the code for Nehalem. We demonstrated that Green Flash could match GPU performance for RTM codes, and offer a 20x energy efficiency benefit over Nehalem and a nearly 10x energy efficiency benefit over the highly optimized GPU implementation.

\section{Hardware Design}

In November 2009 we demonstrated a chip design with multiple cores running the climate model after expanding our simulation capabilities to emulate an entire multi-processor chip design. In 2010, we refined the model for on-chip networks because it is an important differentiator for future chip designs. We have developed infrastructure to incorporate DRAMsim-2 to improve our modeling of chip memory power consumption. Overall, these advances improve our ability to predict the impact of our design decisions on power consumption and chip cost.

\section{CoDesign for Exascale Computing (DOE/ASCR)}

CoDesign is central to our assertion that a holistic approach that combines algorithm design, hardware, and software tuning together enables a substantial improvement over business-as-usual approaches to HPC system design. We have refined our co-tuning and CoDesign methodology to make the Green Flash infrastructure more useful for the broader DOE Exascale Computing initiative. Energy efficiency and hardware/software co-design, which been central to the Green Flash approach, are now broadly adopted by DOE/ASCR as the underpinnings for its multi-billion dollar program to develop an exaflops-scale computing system by 2018 . 
Reference Benchmarks for the Dwarfs

Principal Investigator: Erich Strohmaier, HPCRD

Project Description

For decades, computer scientists have sought guidance on how to evolve architectures, languages, and programming models in order to improve application performance, efficiency, and productivity. Unfortunately, without overarching advice about future directions in these areas, individual guidance is inferred from the existing software/hardware ecosystem, and each discipline often conducts their research independently assuming all other technologies remain fixed. In today's rapidly evolving world of on-chip parallelism, isolated and iterative improvements to performance may miss superior solutions in the same way gradient descent optimization techniques may get stuck in local minima.

To combat this, we are developing a testbed of benchmarks representing important groups of algorithms known as 'dwarfs'. Each dwarf captures a pattern of computation and communication common for a class of important applications. Thus, each dwarf represents a class of algorithms with similar properties and behaviors. This testbed is designed to replace existing benchmarks used in research and to serve as a focal point of research for years to come. It is designed to enable research in a variety of areas such as on-chip and off-chip computer architectures, energy efficient computing, parallel programming paradigms, compilers, and system level software. To avoid preordaining parallel codes, we are developing pencil and paper description for each algorithm, and create realistic, scalable problem descriptions and input datasets to enable experiments from single sockets up to full scale HPC systems. We are providing means to verify proper and accurate program execution and we are also developing reference implementations in high-level languages to demonstrate the algorithms in a simple fashion.

Accomplishments

We assembled and publically released a first full version of the TORCH: Testbed for Optimization ResearCH. It contains a total of 37 kernels representative for most of the scientific computing dwarfs (dense and sparse linear algebra, finite difference methods on structured grids, spectral methods, particle methods, monte carlo methods, graph computations, and sorting algorithms). For all included kernels we created domain-appropriate high-level definitions of the problems solved. To ensure future endeavors are not tainted by existing implementations, we specified the problem definitions to be independent of both computer architecture and existing programming languages, models, and data types.

TORCH includes straightforward, illustrative reference implementations for all kernels developed for clarity and simplicity in MATLAB and C. It also contains scalable problem generators to accompany each computation and routines to access accurate and correct execution of implementations. This allows experimentation with various problem sizes independent of code implementations or mappings to architectures.

To evaluate the utility of our testbed we conducted auto-tuning projects for dwarfs previously not covered. In particular we explored time and memory saving opportunities in a plasma fusion code (GTC), which belongs to the class of the particle methods. One of the most challenging kernels in this code is the particle-to-grid interpolation step for which we demonstrated substantial potential for memory savings on multi-core processors. These savings are only achievable though radically different code re-implementations, which confirm the necessity of hardware and programming model agnostic testbeds such as TORCH for research of this type. 


\section{Managing Petascale Data with Emerging Computer Architectures \\ Principal Investigators: Kesheng Wu, Kamesh Madduri}

\section{Project Description:}

The goal of this research project is to investigate new parallel, memory-efficient approaches for large-scale data management and analysis. Our efforts focus on compact data representations, exploiting concurrency and locality in computation, and a careful orchestration of data access patterns of analytics routines. By effectively harnessing emerging multicore architectures, we design scalable tools for scientific data analysis. We will also investigate new approaches to solve combinatorial graph-theoretic problems at scale, as this class of computations is becoming increasingly important in data analysis. In addition to large data volumes, data heterogeneity, variable data quality, and temporal analysis requirements are other formidable challenges to consider in the design of scalable analytics routines. Our objective is to develop algorithms that encompass several data-centric parallel performance optimizations, and that are applicable to multiple application domains.

\section{Accomplishments:}

Our research contributions to date fall into three broad areas: scientific data management using compressed bitmap indexes, novel parallel graph algorithms for data analysis, and speeding up communication-intensive analytics in emerging science domains, specifically, this LDRD led to new collaborations on data-intensive genomics and cosmology projects.

We designed new algorithms to speed up evaluation of 'ad-hoc range join' queries [CIKM '09]. Our algorithms use bitmap indexes to represent compactly the join output, and achieve significant performance gains over competing approaches. For instance, our count-only algorithm is up to three orders of magnitude faster, and our best bitmap index-based algorithm is 1.2--80X faster than the commonly-used sort-merge algorithm for various test query instances. In collaboration with the visualization group, we have been exploring accelerating search queries on multicore architectures and GPUs [SSDBM '09] using new data structures. We also recently performed an experimental study to evaluate the impact of flash storage devices on the design of bitmap indexes [IDEAS '10]. We observe that multi-level indexing strategies on flash drives are up to twice faster than traditional approaches on magnetic disk drives.

The modeling and analysis of massive and dynamically evolving semantic networks raises new and challenging research problems. Due to their large memory footprint, fine-grained computational granularity, and low cache locality, massive graph problems pose serious challenges on current parallel machines [SIAM AN10]. We presented the first study of parallel combinatorial techniques for analyzing large-scale information networks, encapsulating dynamic interaction data in the order of billions of entities [IPDPS '09]. In this work, we designed data structures to represent dynamic interaction networks, and presented new algorithms for dynamic connectivity problems. We also designed a new lock-free parallel algorithm for computing betweenness centrality [MTAAP '09], a key computational metric in the study of complex networks. In recent work, we presented a linear-time algorithm for single-source shortest paths (SSSP) problem [JDA '10], and a practical high-performance approach for the problem of detecting negative cycles in graphs with arbitrary integer edge weights [AMC '10]. These algorithms are key building blocks in combinatorial optimization, and have applications in chemical informatics, nanotechnology, route planning, and social network analysis. 
Computational Techniques for Non-crystalline X-ray Diffraction Imaging
Principle Investigators: Chao Yang (Computational Research Division), Stefano Marchesini (Advanced Light Source)

The major goal of this project is to develop computational techniques and tools that can be used process diffraction imaging data to elucidate 3D structures of nanomaterials and biological molecules. Non-crystalline X-ray diffraction imaging (Miao, 1999) provides a complementary technique for revealing molecular or cellular structures that are difficult to obtain through other existing techniques such as X-ray crystallography, X-ray tomography, and electron microscopy. Since this imaging technique does not rely on building high-quality lenses or growing crystals, it is suitable for structure studies using a wide variety of light sources including the Next Generation Light Source that is under preparation at LBNL.

Accomplishments:

In the last fiscal year, we have focused on developing and experimenting with algorithms for determining relative orientations of diffraction images to be collected in single molecule diffraction experiments. There are two main approaches we have looked at. The first approach, which requires computing the eigenvectors associated with the largest eigenvalues of a symmetric matrix, is suitable for determining orientations of diffraction images of isolated objects targeted in the original proposal. The construction of the matrix in this approach requires us to identify common curves shared by pairwise diffraction images in the reciprocal space. We developed a technique for identifying these common curves by sampling each diffraction image in a systematic and efficient manner. Using these identified common curves, we were able to obtain the correct relative orientations of simulated diffraction images. We have published the result in the Proceedings of the SPIE conference on Optics and Photonics. The second approach, which involves using compressive sensing techniques to solve an L1 minimization problem, is suitable for nanocrystals that consist of roughly tens of unit cells. The reason why we have broadened our scope in the original proposal to consider nanocrystals is that the first set of data that have become available at LCLS contains only diffraction images of nanocrystals of Photosystem I (PS1). For crystals, the orientation determination problem is known as the indexing problem. We have examined the existing algorithm for solving the indexing problem in CCTBX. Such a program typically requires a larger number of visible Bragg peaks in the diffraction image to reliably determine crystal unit cell parameters and orientations. For nanocrystals that have fewer unit cells and weaker Bragg spots in diffraction images, compressive sensing, which in principle does not require a large number of peaks to be present, provides a natural alternative to the existing indexing algorithms. We formulated the indexing problem as a basis pursuit problem and applied efficient optimization algorithm to recover the crystal lattice from as few as 3 Bragg peaks of simulated data. We published our technique and results in the Ultramicroscopy journal.

In addition to solving the orientation determination problem, we have also begun the investigate numerical techniques for phase retrieval. 


\section{LB09035}

\section{Earth Sciences Division}

Uncovering The Mechanistic Basis For Soil Microbial Community Response To Altered Precipitation Patterns. Principal investigator: Eoin Brodie

Project Description

The goal of this LDRD is to further our understanding of the mechanisms by which soil microbial communities respond in composition and function to altered precipitation patterns. This project will significantly increase our understanding of how anticipated changes in rainfall might affect terrestrial biogeochemistry, particularly soil carbon cycling. We are studying these processes using laboratory and field rainfall manipulations of soil microbial communities across three geographically distinct environments with contrasting precipitation regimes (desert, Mediterranean grassland and tropical rainforest). These three soil microbial populations have evolved under different rainfall regimes allowing for an evaluation of the evolutionary constraints upon organismal and community adaptation to climate change. The workflow takes a bottom-up, three-pronged approach to this question by focusing on the properties of individual organisms, microbial communities and the biogeochemical processes they mediate.

\section{Accomplishments}

We have developed a pipeline for the preparation of amplicons for multiplex high-throughput sequencing. This encompasses the extraction and amplification of 16S rRNA from DNA/ RNA extracted from soil and the subsequent purification and concentration of PCR for sequencing. These methods have been used to analyze changes in microbial community structure in response to manipulated rainfall. Overall we have found that community phylogenetic diversity decreased with declining precipitation. Such biodiversity loss may have important implications for ecosystem function. We further examined the effect of altered precipitation patterns using a microcosm experiment with tropical rainforest soils collected from control and rainfall exclusion plots. Following simulated precipitation, trace gas data demonstrated a reversal from sink to source for $\mathrm{N}_{2} \mathrm{O}$ where soils that had previously experienced decreased rainfall emitted $\mathrm{N}_{2} \mathrm{O}$ while controls soils consumed $\mathrm{N}_{2} \mathrm{O}$. We are currently sequencing the microbial community at successive time points to determine whether shifts in microbial composition or functional expression are responsible.

We have also succeeded in establishing a successful culturing pipeline for targeting Actinobacteria from the three distinct ecosystems. Actinobacteria are functionally important organisms in the degradation of recalcitrant soil carbon pools and this study furthers our understanding of their genetic capacity to adapt to a changing climate. To date we have carried out a number of water potential stress studies to simulate decreasing precipitation including culturing isolates across a gradient of water potentials. Isolates from the three ecosystems displayed varying water potential optima that were related to their climate histories. We have also obtained draft genome sequences for a collection of Actinobacteria from the tropical and Mediterranean grassland soils which will be used to map mRNA-seq transcriptome data following water potential manipulations. Finally, we have developed a methodology for efficient cellular lysis and protein extraction from soils to directly examine the proteome of soil microbial communities undergoing rainfall manipulation and are complementing this with analysis of isolate and community metabolomes under a systems biology approach. 


\title{
Enhanced Subsurface Fluid Characterization Using Joint Hydrological and Geophysical Imaging
}

\author{
PI(s): Michael Commer, Stefan A. Finsterle, Michael B. Kowalsky, and Gregory A. Newman
}

\section{Project description}

The goal of this project is to develop approaches that advance the understanding of fluid flow and transport in complex geologic formations by combining hydrological and geophysical measurements into a joint imaging (or joint inversion) framework. Such approaches are needed to improve hydrological state and parameter estimation in applications such as geothermal reservoir characterization, environmental remediation monitoring, geologic disposal of heatgenerating nuclear waste, and $\mathrm{CO}_{2}$ sequestration.

Quantitatively assessing and predicting physical and chemical processes that control subsurface flow systems often demands high-resolution characterization data on multiple scales, which can be provided economically by geophysical measurements, complementing typically sparse hydrological well log measurements. Our imaging approaches involve a progressively stronger coupling of two established technologies developed at LBNL for joint hydrogeophysical characterization on a field scale, where geophysical, particularly electrical and electromagnetic, data are combined with hydrological data for high-resolution three-dimensional mapping of the subsurface parameters (e.g., permeability) and state (e.g., fluid distribution).

\section{Accomplishments}

We made progress on one inversion strategy that uses geophysical images to constrain hydrological inversions. A nonlinear least-squares optimization is performed using a geostatistical technique with pilot points to reduce the dimension of the parameter space. Using synthetic modeling studies based on tracer transport in a shallow aquifer, we demonstrated how the subsurface permeability distribution can be improved by including electrical resistivity tomography data.

In order to handle large-scale earth models, we completed enhancement of the LBNL code iTOUGH2, which provides inverse modeling capabilities to the flow and transport simulator TOUGH2, so that it can run on parallel computing systems. Together with LBNL's parallel nonlinear conjugate gradient (NLCG) based geophysical imaging scheme EMGeo, the 3D joint imaging framework now has the capability to carry out inverse modeling, optimization, and uncertainty analyses of voluminous hydrogeophysical data sets with large spatial coverage, using massively parallel computers.

So far we have also demonstrated the applicability of different components of our inversion methods using field data from DOE's Rifle site $(\mathrm{CO})$, where experiments are underway to examine the potential for monitoring microbial interactions in a uranium-contaminated aquifer. We developed a crucial component for the geophysical inversion that consists of a NLCG parameter-weighting scheme, which achieves a focusing effect for geophysical surface measurements. In addition, a hydrological model was developed to simulate the concentration measurements collected in a suite of observation wells, so that the heterogeneous distribution of permeability and porosity can be estimated through a geostatistical approach. We are currently investigating the improvement of the analysis by incorporating geophysical data with different joint inversion approaches. 


\title{
Carbon Uptake and Partitioning in Plants and Algae
}

\author{
Principal Investigator: Christer Jansson
}

\section{Project description}

The ability of plants to convert solar energy into biomass is of immense importance since the very survival of both photoautotrophic and non-photoautotrophic organisms depends on this process. In addition, the efficiency of photosynthetic $\mathrm{CO}_{2}$ assimilation into storage and structural products by the plant is one of the main factors that determine the economic viability of biofuel production, and is also crucial for terrestrial carbon sequestration. The overall aim of the proposed project is to study the interactions of photosynthesis in leaves (source) and the synthesis of storage and structural carbohydrates (sinks) in cereals such as barley, sorghum and rice and in storage root crops like cassava, and impact of such interactions on biofuel production and terrestrial carbon sequestration.

\section{Accomplishments}

We have reviewed the prospects of plant genetic engineering in enhancing terrestrial carbon sequestration (phytosequestration) in the coming 50 years. In so doing, we give examples by which genetic engineering can contribute to phytosequestration, and we made efforts to quantify such strategies. The BioScience article where the review was published received high media coverage.

We have applied transcriptomic analyses to investigate stress responses in cereals imposed by increased temperature using barley as a representative plant. Employing the Affymetrix 22K Barley1 GeneChip microarray we found 958 genes to be induced and 1122 genes to be repressed by heat stress. The genes exhibited spatial and temporal expression patterns that provide a detailed insight into the caryopses' early heat stress responses. Down-regulation of genes related to storage compound biosynthesis and cell growth provides evidence for a rapid impairment of the caryopsis' development. Increased levels of sugars and amino acids were indicative for both production of compatible solutes and feedback-induced accumulation of substrates for storage compound biosynthesis. Metadata analysis identified embryo and endosperm as primary locations of heat stress responses, indicating a strong impact of short-term heat stress on central developmental functions of the caryopsis. Summarized, our data provide an important basis for further investigation of gene functions in order to aid an improved heat tolerance and reduced losses of yield in barley as a model for cereal crops.

We have characterized biomass production in cassava and evaluated it's potential as a bio fuel crop in China. We have identified and examined the expression profile for one gene of critical importance for biomass production in the cassava storage root. 
Microbiomics of Complex Microbial Communities in Environmental Samples Principal Investigator: Janet Jansson

\section{Project description}

Advances within the emerging field of "microbiomics" and other "omics" fields have made it tractable to characterize entire microbial communities and describe their individual components as well as their roles in various biogeochemical processes and interactions with the environment and man. The aims of my research, all using "omics"/systems biology approaches, are the following:

1) Characterization of the soil microbiota, with emphasis on permafrost - relevance for climate change and biosequestration

2) Characterization of sediment microbiota - relevance for bioremediation and biogeography

3) Characterization of the human gut microbiome - relevance for disease biomarkers and drug targets; with a focus on Crohn's disease

Accomplishments

One of our main accomplishments was to perform deep metagenome sequencing of the permafrost soil microbiota in samples collected from two sites in Alaska. This project, in collaboration with JGI, represents one of the biggest sequencing projects to date, with billions of base pairs of data. The sequence coverage was sufficiently great to enable assembly of a draft genome of a novel methanogen. In addition, we determined the composition of the soil microbial community by sequencing of hundreds of thousands of signature DNA sequences in permafrost samples before and after thaw. We found that there is a rapid shift in the permafrost microbial community after a short-term thaw. A manuscript was written and submitted to Nature.

We also developed a protocol for extraction of proteins from soil and demonstrated its applicability for identification of thousands of proteins from bacteria inoculated into soil, as well as native soil bacteria. This protocol was published and is now being applied to study other soil ecosystems as part of a recently funded DOE-BER grant focused on the soil carbon cycle.

We collected contaminated sediments from the Northern hemisphere (Sweden and Norway) and the Southern hemisphere (Argentina and Antarctica) and extracted DNA from those samples. They are currently being sequenced at JGI as part of a newly funded sequencing project. We also published a series of studies of the impact of pollution on bacterial communities in Baltic Sea sediments.

We developed a new method for comparing matched metagenomes and metaproteomes and used this approach to characterize the gut microbiota in a set of Swedish twins with Crohn's disease. The use of twins enabled us to specifically focus on the contribution of the microbial community to Crohn's disease, independently of host genetics, since both are known to play a role. We found specific metabolic biomarkers of Crohn's disease that was the basis for an invention discovery. In addition, we identified specific bacteria and proteins that were correlated to Crohn's disease. We are extending these studies to look at the dynamics of the intestinal microbiota over time in individuals with Crohn's disease in a newly funded NIH project. 


\section{Impact of Climate Change on Soil Water Dynamics in Arid Areas \\ Principal Investigator(s): Hui-Hai Liu}

\section{Project Description}

Understanding soil-water dynamics in arid areas is critical for the management of a number of U.S. Department of Energy (DOE) sites, including the Nevada Test Site and Yucca Mountain Site in Nevada and the Waste Isolation Pilot Plant (WIPP) Site in New Mexico. The potential contaminant transport at these DOE sites is largely determined by soil-water dynamics (such as groundwater recharge). However, the impact of climate change on soil water dynamics in arid areas is not well understood at this point, because of the difficulties in accurately measuring soil-water flow processes under arid conditions and in quantifying complex interactions among climate, soil water, and vegetation in these areas.

The overall purpose of this study is to develop an innovative approach to assessing the impact of climate change on soil-water dynamics in arid areas. This approach is based on a new hypothesis: in arid areas, temporally averaged soil-water saturation will not be affected by climate change during growing seasons as a result of the naturally occurring optimization processes for vegetation growth. We will evaluate this hypothesis with data from literature and develop a stochastic modeling method based on the hypothesis.

\section{Accomplishments}

Results from a comprehensive literature survey of measurement soil-water data support our hypothesis. Using the hypothesis, we developed a local-scale stochastic (probabilistic) modeling method for interaction among climate, soil water, and vegetation in arid areas. We have applied the method to study impact of climate change on soil water recharge in Yucca Mountain area in Nevada and found that the recharge variation is significantly buffered by changes in vegetation coverage. We also found that in addition to mean soil-water content in root zone, soil-water recharge is mainly determined by frequency of heavy rainfalls associated with climate changes.

Especially, we have focused on investigating the relative importance of climate change on shrub expansion in arid areas based on the concept of the optimum soil-water saturation. Shrub expansion in arid areas, which has been reported worldwide, has significant ecological and economical implications. Our study shows that extreme precipitation events due to climate change contribute to the shrub expansion in the arid areas. 
$\mathrm{CO}_{2}$ as Cushion Gas for Compressed Air Energy Storage in Subsurface Reservoirs Principal Investigator: Curtis M. Oldenburg

\section{Project Description}

We are investigating the use of $\mathrm{CO}_{2}$ as a cushion gas for Compressed Air Energy Storage in aquifers and in depleted gas reservoirs (Aq-CAES, and DR-CAES, respectively). The advantage of using $\mathrm{CO}_{2}$ as a cushion gas is that $\mathrm{CO}_{2}$ compresses in a non-linear and advantageous fashion such that it acts like a super-cushion when the reservoir is operated around the critical pressure of $\mathrm{CO}_{2}$ (74 bar). The main goal of the project is to assess the feasibility of using $\mathrm{CO}_{2}$ as a cushion gas for enhancing energy storage, while also developing expertise in the geoscience (hydrology, geochemistry, geomechanics) of CAES.

The approach we are using involves simulation of the coupled processes involved in AqCAES. Many of the processes associated with $\mathrm{CO}_{2}$-enhanced Aq- and DR-CAES can be modeled with reservoir simulation capabilities we have developed (e.g., T2Well (Pan), and TOUGH2/EOS7C (Oldenburg)). Through use of existing simulation capabilities, we are investigating processes of air injection in the well and in the reservoir including the effects of repeated compression and decompression cycling and displacement of water.

\section{Accomplishments}

We developed a prototype 2D radial Aq-CAES reservoir that is operated with the same energy storage and injection parameters as the existing salt-cavern CAES facility in Huntorf, Germany. The Aq-CAES reservoir we model is $50 \mathrm{~m}$ thick, $10 \mathrm{~km}$ in radius, and has isotropic permeability of 1 Darcy with porosity of $20 \%$. We have modeled the details of energy and mass flow in the 21-inch well and in the reservoir during the creation of the initial gas bubble over 15 days ( 360 hours) and followed by 10 daily cycles of energy storage (air injection and production). Results for standard Aq-CAES (no $\mathrm{CO}_{2}$ cushion) are presented in Figure 1. We find that the roundtrip efficiency is approximately $90 \%$, not including the air-compression or electricity generation steps. Losses arise in the CAES system from heat loss in the well and long-term water displacement in the reservoir. During FY11, we will add $\mathrm{CO}_{2}$ as a cushion gas a compare results against this base case.

(a)

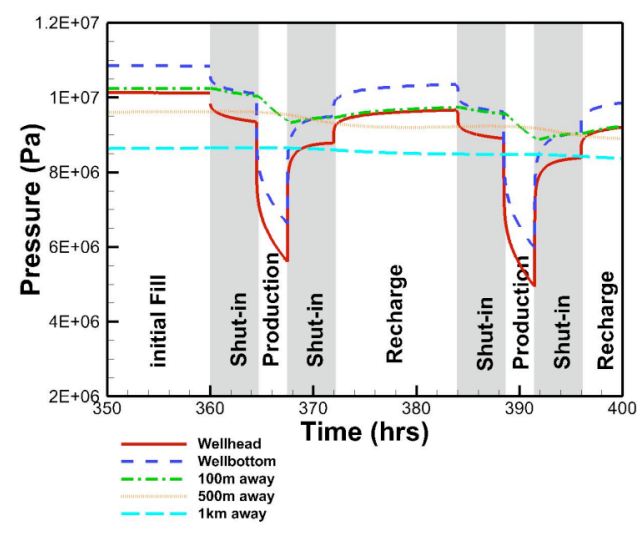

(b)

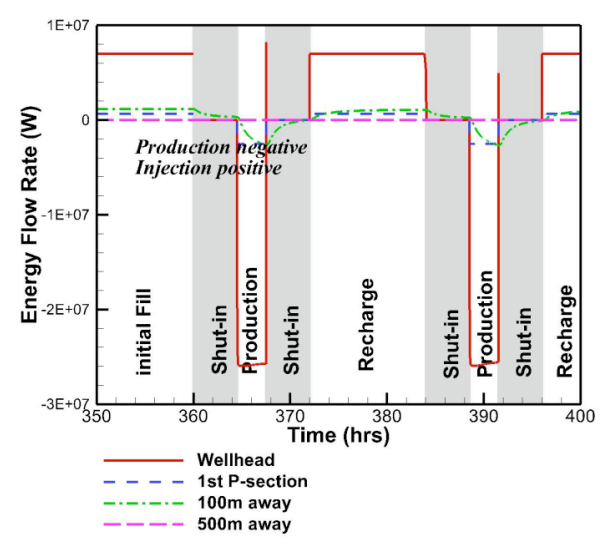

Figure 1. Results of Aq-CAES showing two cycles of storage and production by (a) pressure and (b) energy flow rate versus time. 


\section{Identifying and Predicting Climate Change Impacts on the Land-Based Components of the Water Cycle \\ Principal Investigators: Rohit Salve, William E. Dietrich, and Stefan Finsterle}

\section{Project Description}

Our goal through this effort is to (1) document the importance of the fractured rock continuum that underlies most landscapes in regulating the hydrologic cycle and (2) determine how the hydrology of this zone can be impacted by climate change. We are using an unprecedented array of deep and shallow water-level and moisture-detection devices to document how pulses of rainwater pass through hillslopes, and how residual water is removed either through deep subsurface flow, plants, or transpiration during critical drying periods. We are also exploring the use of repeat geophysical surveys to detect moisture change in the deep subsurface. Key here is to identify and develop techniques to predict the influences related to meteorological and hydrological events, which may vary as climate may vary in the future.

The hypothesis of this proposal is that the underlying fractured rock in sloping landscapes regulates both the quantity and quality of water that becomes available to downslope ecosystems. An understanding of the physical makeup of this fractured continuum, and how water is stored and transmitted through it during and between precipitation events, will lead to improved predictive capabilities of trends in water quality and quantity, as dictated by changes in climate.

\section{Accomplishments}

We have now collected high-resolution (temporal and spatial) measurements of the underlying water table at multiple locations within the watershed, and continuous measurements of saturation changes close to the surface that extend over a period more than two years. In addition we have completed a series of measurements of saturation changes in the underlying bedrock with neutron logging and electrical resistance tomography. This data is now being analyzed to explain the distinctive moisture signals observed along the expected flow path. Numerical simulations have been initiated to test various hypotheses regarding water transport from the land surface through the top soil and the fractured mudstone to the water table. Findings from this investigation are being presented to the scientific community during conferences and through publications.

Our results suggest that the underlying fractured rock is not only the predominant pathway for water flow through this hillslope, but it also serves as reservoir for water that likely sustains the dense vegetation during the long dry summer season. In addition, the geophysical measurements along with the observed well water responses show that there is significant variability in the hydrologic properties of the fractured rock which results is a range of response patterns to individual rainfall events. These observations will help constrain our numerical model for conditions anticipated for climate shifts towards drier conditions. 
Biological Carbon Sequestration at Berkeley Lab

Fundamental Research on Biological Carbon Capture and Soil Carbon Stabilization

Principal investigator: Margaret Torn

Project description

The purpose of this project is to study and optimize biological means to capture and sequester $\mathrm{CO}_{2}$. Biological capture of $\mathrm{CO}_{2}$ and stabilization in soils is promising as a climate change mitigation approach because some forms of soil organic carbon can persist undisturbed for long periods of time. Our general aims are: (1) to understand mechanisms of soil $\mathrm{C}$ stabilization and how to influence them; (2) to understand how to enhance or influence different means of biologically capturing $\mathrm{CO}_{2}$ in ecological and industrial environments; and (3) to understand and evaluate strategies to minimize loss of already sequestered terrestrial C. Another aim is to integrate Berkeley Lab expertise in the area of biological carbon cycling and biosequestration into the Berkeley Lab Carbon Cycle 2.0 Initiative.

\section{Accomplishments}

The first task was to investigate the carbon stabilization and soil sequestration potential of pyrolized plant material (biochar). We performed incubation experiments by addition of biochar to a grassland soil and we monitored the microbial community response using a combination of molecular approaches. We obtained hundreds of thousands of signature DNA sequences for microorganisms in the samples that were incubated with and without biochar addition. We also performed enzyme assays to determine the functional response of the microbial community to biochar. This study enabled us to determine which microorganisms in soil responded to biochar and how that response varied over the incubation period. These data were correlated to soil carbon analyses and a second experiment using biochar that has been labeled with stable isotopes has been initiated. A manuscript was completed based on results of the first set of experiments.

The second task was to investigate the ability of cyanobacteria to take up carbon (for example from flue gas) and transform it into recalcitrant organic compounds or to mineralize it to $\mathrm{CaCO}_{3}$. We have established synchrotron radiation Fourier transform IR (SR-FTIR) as a powerful tool to study calcification in individual cyanobacterial cells. We are currently developing flow cell FTIR technologies that will allow us to follow the calcification process in real time, and secondary ion mass spectrometry (SIMS) methods to characterize the extracellular polysaccharide outer membrane surface properties and their influence on $\mathrm{CaCO}_{3}$ crystal nucleation and formation. Using bacterial display we have identified candidate peptides for enhancement of $\mathrm{CaCO}_{3}$ nucleation on bacterial cell surfaces. Genes for selected peptides will be introduced into cyanobacteria. Metabolic flux analyses of calcifying cyanobacteria using time-of-flight (Tof) secondary ion mass spectroscopy (Tof-SIMS) and SR-FTIR are ongoing. One manuscript was published describing the potential for calcifying bacteria as a means for point source carbon capture and sequestration. 


\title{
Engineering Division
}

\author{
Advanced Si Detectors for Future Short Pulse X-ray Sources
}

P.I.: Marco Battaglia and Peter Denes

Project Description

The development of future X-ray sources, high energy electron accelerators, as well as accelerators for hadrontherapy, requires detectors of new design to provide efficient beam monitoring and diagnostics. Requirements involve both operation in very high particle fluence for the primary beam monitoring and high precision and fast readout detection of soft particles. The short, intense pulses which they generate are difficult to image with conventional methods. We develop new pixellated Si sensors based on innovative semiconductor technologies and apply them in experiments under realistic conditions at the ALS and LOASIS.

Accomplishments

The two main accomplishments of the project during FY10 are i) the design, production and test of a Silicon-On-Insulator (SOI) pixel sensor immune from back-gating effects and able to operate up to 70V and ii) the successful development and test of SOI sensor back-thinning and post-processing to extend the sensor sensitivity to low-energy radiation, iii) systematic studies of betatron radiation in plasma acceleration using a calibrated CCD system for X-ray spectroscopy on the LOASIS beam line. One of the main outstanding issues in the development of SOI pixels sensors for X-ray and particle detection so far has been the back-gating effect, which limits the depletion voltage applicable to the high-resistivity substrate and thus the thickness of the sensitive volume. A new pixel design, developed in collaboration with KEK, uses a buried p-well to insulate the readout electronics and demonstrated to be immune from back-gating. A test pixel sensor has been successfully tested at depletion voltages up to $100 \mathrm{~V}$. Two beam tests have been performed, with mono-energetic X-rays at the beam-line 5.3.1 of LBNL ALS and with $200 \mathrm{GeV}$ pions at the SPS North Area at CERN, Geneva, Switzerland. The beam test at CERN has been carried out in collaboration with UCSC, INFN and KEK. The tests have provided a characterisation of the response to X-rays and high energy charged particles. In particular a detection efficiency for $200 \mathrm{GeV}$ pions in excess of $98 \%$ and a single point resolution of $\sim 1$ micron using a 14 micron pixel. In parallel with the sensor development an R\&D program on thin SOI sensor back-plane post-processing to create a thin entrance window has been carried out. A low-temperature phosphor implant performed by the group of Craig Tindall in the Engineering Division at LBNL has been successfully tested on the new SOI sensors. The lab characterisation of sensors thinned to 50 microns and with the phosphor implant on the back-plane has given a good yield of functioning sensors. The post-processed SOI sensors are being applied to the detection of $20 \mathrm{keV}$ electrons for beam monitoring through the electron surface emission technique (SLIM). These result open new perspectives for the application of SOI pixel sensors to the detection of soft X-rays and electrons, topical to this LDRD program.

The activity on SOI pixels for X-ray and particle detection, which emphasises fast readout and spatial resolution has been complemented by the use of the LBNL thick, high-resistivity CCDs for highresolution X-ray spectroscopy for monitoring in plasma beam acceleration. The spectrum of betatron X-rays produced by electrons oscillating in the focusing field of the plasma provides important information on the electron acceleration in the plasma. CCD sensors are well suited for this application, pioneered in this LDRD project. The high pixellisation allows us to isolate a pure sample of individual $\mathrm{X}$-rays despite the large electron and photon flux. The CCD high energy resolution ensures a precise spectrum reconstruction. Results of the experiments carried out at LOASIS, where the energy of betatron X-rays measured over their full spectrum in the CCD demonstrates a correlation with the electron energy and the laser parameters, have been published. 
Parallel Microfluidic Synthesizer: A Fully Automated Chemical Evolution Platform for Novel Materials Discovery

Principal Investigator(s): Rafael Gomez-Sjoberg

Project Description

This project aims to develop a parallel microfluidic organic synthesizer capable of fully automated, directed evolution of novel polymeric compounds. This instrument combines both chemical synthesis and biological assay functionalities on a single platform. It can perform automated iterative cycles of parallel chemical synthesis and compound evaluation, in which the assay results inform the design of the chemical synthesis step in each iteration by the use of optimization algorithms, to converge on compounds with desired properties.

Such a device would be immensely useful, and even revolutionary, for a variety of applications: It would allow researchers to rapidly identify robust chemical ligands to biological molecules, discover novel self-assembling nanomaterials, and identify materials with unique optical properties. Because of the broad applicability of this platform, it will operate in the Molecular Foundry and be available to users. Thanks to the use of microfluidic technologies, the system will consume small amounts of reagents and generate small amounts of hazardous waste, compared to traditional synthesis machines. Thanks to high levels of automation, the evolution instrument under development will be able to run continuously at low cost - sifting through sequence space around the clock until a hit is found.

As an initial application we will employ the device to evolve peptoid ligands to protein targets.

\section{Accomplishments}

The most significant accomplishment has been the development of novel fabrication protocols for microfluidic devices made of glass and Teflon. These devices have microfabricated valves incorporated into the microfluidic network, providing totally automated control of fluid flow inside the device. The devices must be made of glass and Teflon because traditional microfluidic technologies that incorporate valves use materials that would not be compatible with the reagents used for chemical synthesis. We have optimized several aspects of the glass-Teflon fabrication process, and have tested a variety of valve designs. We are able to fabricate fully functional devices with a multitude of integrated valves, and have tested

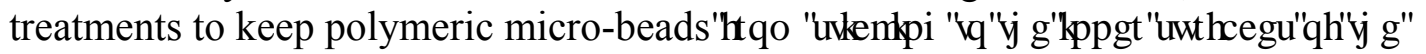
microfluidic channels during synthesis experiments. We have also developed and tested microfabricated structures that can retain the beads during synthesis, but can be opened to release the beads at the end of a synthesis run.

We are currently testing actual peptoid and peptide synthesis procedures in the microfluidic devices, and refining the design of these devices for optimal synthesis performance. 


\section{High-Temperature Superconductors for Compact X-ray FEL's Principal Investigator(s): Soren Prestemon}

\section{Project Description}

The purpose of this project is to demonstrate a novel prototype undulator device using YBCO tape superconductor that holds promise for very high performance at short periods, providing users with access to short-wavelength radiation. Prototypes will be designed, built and tested at LBNL to demonstrate attainable field and develop fabrication techniques. Transport current will be measured, and current distribution profiles analyzed.

As part of the development of such a technology, we will provide analysis of tolerances and error fields, and their impact on undulator spectral performance. Methods to mitigate field errors will be studied. A preliminary investigation into wakefield limitations will be performed.

The impact of the technology on light-source beam energy selection and system cost will be evaluated. Applicability to a variety of light source systems, including FEL "farms" and "Tabletop FEL's", e.g. using plasma acceleration, will be studied.

Accomplishments

The major accomplishment attained through this LDRD include:

1) The development of a lithography process that allows for fast, cheap, and highly accurate fabrication of the core conductor elements that form the basis of the undulator. This process has been optimized and can now be readily applied for various period designs as needed.

2) The design of a support structure for a full-scale prototype has been completed, and a sample support structure was fabricated using rapid prototyping plastics to allow simple testing of the concept.

3) The initial development of a magnetic measurement system that should allow for accurate measurement of field errors when a full-scale prototype is operational. The measurement system is based on a pulsed-wire concept that can be made compatible with the very small gaps $(\sim 2 \mathrm{~mm})$ and cryogenic, in-vacuum regime of operation of the device. This is a critical element of any superconducting undulator development, as existing techniques are unable to provide sufficiently accurate field measurements.

4) Development of a basic analysis approach for tolerances and field errors, which can be used to quantify allowable fabrication errors for device components. The nominal approach to field error mitigation is through accurate fabrication and assembly processes; approaches to provide post-fabrication corrections are still being investigated.

5) An initial look at the impact of such devices on accessible wavelength spectrum in conjunction with beam energy has been performed; the performance is particularly attractive for hard $\mathrm{x}$-ray applications requiring fairly modest tuning range, since this device may provide such x-rays with fairly low beam energy, resulting in a low overall system cost; alternatively, very short period undulators may provide a means of accessing hard x-rays from soft x-ray FEL systems. 
LB09003

\section{Environmental Energy Technologies Division}

Embedded Engineering, Construction Materials, and HVAC Components in Modular Energy

Systems Simulation

Principal Investigator: Vladimir Bazjanac

Project Description

The project had two goals: (1) to define principles and begin developing tools essential to component based Building Energy Performance (BEP) simulation that can be instantiated and parameterized from a Building Information Model (BIM); and (2) to incorporate applicable principles of information science to the process of using BEP simulation and analysis by the Architecture-Engineering-Construction-Operator-Owner (AECOO) industry.

Project work created initial conditions needed to change the current BEP simulation practice, and contributed to the development of an environment that will facilitate the design of buildings with embedded engineering, construction materials and energy efficient components. This was accomplished by developing and testing a methodology and software that enable a paradigm change in how BEP simulation software is currently used, and enables direct interoperability with other (interoperable) software that is routinely used in AECOO industry projects.

\section{Accomplishments}

Our major accomplishment was the development of a methodology for semi-automated BEP simulation and the implementation of automated simplification of building geometry. Geometry Simplification Tool (GST), a prototype translator that automatically extracts data from BIM, transforms extracted data as necessary per rules embedded in software, and automatically generates geometry input for BEP simulation with EnergyPlus. GST can be appended to automatically generate geometry input for other simulation and analysis tools which require building geometry to operate. We completed and tested GST Version 1.2 and published GST test procedure and results. GST is already used in the implementation of the pending GSA requirement to include BIM-based BEP simulation results in the documentation of Final Concept submissions for federal buildings (GSA project Concept Design BIM 2010). GST is also being incorporated in project Mojito (LBNL development of a graphic user interface for EnergyPlus).

We discovered that the current industry practice of Value Engineering (VE) is a major reason why buildings are not as energy efficient as they were designed to be. Many energy-efficiency components and measures are routinely eliminated for first-cost reasons when the long term effects on building operations cannot be quantified during the same VE event. We submitted a still pending proposal to DOE's Buildings Hub that would eliminate this shortcoming by standardizing the VE process and providing interoperability to all software tools used in VE.

Perhaps the most important outcome of this project was the realization that a significant part of data used in the AECOO industry (and not only in simulation) cannot be substantiated - data origin is sometimes unknown, values are often arbitrarily "adjusted" (i.e. approximated or modified), or data are at times simply invented. The industry is constantly subject to the well known computer science dictum GIGO (Garbage-In-Garbage-Out), without being aware of it. This has potentially very serious validity implications not only for the results of simulation and analysis in the industry, but also for industry-related research. Yet, industry and scientific literature are silent about this problem. This prompted the writing of an article that will be submitted to journal Science by the end of April 2011. It discusses why buildings perform worse than predicted, data transformation that does not meet standards of science, results of simulation and analysis in the AECOO industry that are not reproducible, the impact of current use of data on the energy performance of buildings, and possible ways to circumvent problems with data. 


\section{Physically-based Accounting for Resource Use in Alternative Energy Pathways Principal Investigators: Katie Coughlin and David Fridley}

\section{Project Description:}

The goal of this project is to develop a new conceptual model for defining resourceallocation schemes under conditions where environmental or other physical constraints may be more significant than economic costs. In developing the methodology, for definiteness we focus on a particular question: how much of the energy demand of the power generation and transportation sectors can be met by a truly carbon-neutral, biomass based system? The requirement of carbon-neutrality provides the most binding physical constraint: to be truly carbon-neutral, the total emissions must be balanced by the annual uptake of carbon by the biofuel feedstocks. Using methods developed in previous work, we construct a physical inventory of the agricultural resources available, and reallocate them based on the requirements for the production of various feedstocks.

Our approach is built on methods used in ecological food web modeling. In ecosystems nature solves an allocation problem, partitioning land, water and biomass resources among a complex assemblage of populations, which are related through both symbiotic and predator-prey dependencies. Food-web analysis partitions the ecosystem into levels, which are defined by who eats whom. The analogy of the food-web here is the energy "production web" which takes in a raw materials and produces a wide variety of products in addition to energy. A proper accounting of alternative fuel production schemes must include the impacts on the entire production web, including possible shortages of nonenergy petrochemical products.

Accomplishments:

Year one activities are organized around refinement of the methodology and collection and assimilation of data. For the methodology, we have developed a rigorous mathematical metric for fossil-fuel use in an arbitrarily complex production chan. Based on a review of the existing literature we have identified the five principal pathways for biomass-to-energy (pyrolysis, fermentation, bio-oil, gasification, direct combustion) and constructed a formal description of these processes that allows us to estimate the maximum possible output based on simple physical constraints.

For data collection, we have compiled four databases:

1. US agricultural output, acreage, irrigation use and yields.

2. A geographical database for baseline agricultural water needs (based on weather data and a model for crop water use as a function of weather variables).

3. A database of agricultural data for energy feedstocks, including crop water use coefficients and crop yields.

4. A database on biomass-to-energy processes, including input feedstock, output fuel, process energy required, process yields, and other resource needs. 
Toward a US Greenhouse Gas Information System

Principal Investigator(s): Marc L. Fischer

\section{Project Description}

We are conducting research and development of methods to quantify anthropogenic greenhouse gas emissions at local, regional, and eventually continental and global scales. The longterm goal of this work is to support international control of anthropogenic greenhouse gas emissions, which will require systematic estimation of emissions and independent verification. In this research component, we are expanding on the LBNL California Greenhouse Gas Emissions Measurement (see CALGEM.lbl.gov) project, which we started for the California Energy Commission.

Accomplishments

This project started as a mid-year LDRD in June, 2010. Hence the accomplishments reported here represent work conducted from June to September.

Our primary research has focused on improving both data and modeling capabilities to make California a uniquely powerful test-bed for measurements of GHG emissions. As proposed, we are engaged in a multi-agency (California Energy Commission, California Air Resources Board, DOE, NOAA, NASA) research intensive called CalNex2010. This LDRD enables us to combine CALGEM and CalNex2010 data, addressing research problems crucial to emissions measurements. Our primary accomplishment has been to complete a set of updated atmospheric transport model simulations for summer 2010 (the CalNex2010 period) that will be used with tower, airborne, and satellite GHG measurements for inverse model analysis of $\mathrm{CH}_{4}$ and other GHG emissions. This is expected to make LBNL a high-value partner for future proposals to collaboratively analyze the CalNex and other California GHG data sets. Secondary research accomplishments include preliminary reduction of a continuous $\mathrm{N}_{2} \mathrm{O}$ from the tall-tower LBNL operates near Walnut Grove, CA. In FY11, we plan to complete a manuscript estimating regional $\mathrm{CH}_{4}$ emissions from Central California using a combination of the above data sets and transport modeling. In FY12 we intend to submit a manuscript estimating California-wide GHG emissions, identifying the role an expanded measurement network in California or at an existing instrumented facility (e.g., DOE-ARM) could serve as a test-bed for emissions measurements. 


\title{
Developing a Fuel-Efficient Cookstove for Haiti
}

\author{
Principal Investigator: Ashok Gadgil
}

\section{Project Description:}

The purpose of this project is to identify and characterize low-cost cookstoves for Haiti that are appropriate for available fuel types and cooking practices, consume less fuel, and emit fewer harmful pollutants than the rudimentary cook stoves and open fires currently prevalent in Haiti. Given the large array of cookstoves planned for distribution in Haiti and the relative lack of comparative data on these stoves, this project seeks to fill the informational gap by performing efficiency and emissions testing on cookstoves that are currently being considered for governmental or non-governmental dissemination in Haiti. The project aims to reduce the negative health and environmental effects resulting from Haitians' widespread reliance on solid fuels for cooking by providing stove projects in Haiti the necessary data and analysis to select stoves that are efficient, have low emissions of harmful pollutants, and are appropriate for local cooking styles and utensils.

Project Accomplishments:

A team of three people conducted a two-week data collection and fact-finding mission to Haiti in the Spring of 2010 in order to determine how LBNL's expertise could most effectively be leveraged to increase the efficiency of the cookstoves used in Haiti. While in Haiti, team members met with field partners, collected data, and tested alternative stove types. Following the fact-finding mission, the team determined that the most useful cookstove-specific role that LBNL could play in Haiti would be to perform high quality laboratory testing of the most common stoves being considered for distribution by NGOs and governmental groups in Haiti. Most of the NGOs that the team met with in Haiti had plans to distribute cookstoves, but very few seemed to have in-depth knowledge of the performance of the stoves that they were considering.

Beginning in July 2010 a team of students, with guidance from the fact-finding team, undertook stove testing on five charcoal stoves being considered for distribution in Haiti using the standard Water Boiling Test (WBT) protocol. Each stove was tested for time to boil, efficiency, specific fuel consumption, and emissions of carbon monoxide and carbon dioxide. The results were analyzed and compiled in a report that is currently being finalized. In October, draft versions of the report were made available to NGOs that had begun choosing cookstoves for dissemination in order to provide them with data to use in their selection process. Upon completion of the WBTs, project members drafted a Controlled Cooking Test (CCT) protocol intended to replicate local cooking styles and conditions as closely as possible in the laboratory setting. In cooperation with other organizations carrying out this type of testing in Haiti, project members have finalized the protocol for Diri Kole ak Pwa, Haitian beans and rice. CCTs began in November 2010 and are anticipated to be completed in January 2011. 


\section{Development of Novel Improved Capacitors for Pulse Power Applications Principal Investigators: John B. Kerr, Venkat Srinivasan and Marca M. Doeff.}

The project has investigated the interfacial behavior of different electrolytes at various electrode surfaces with a view to increasing the energy density of supercapacitors to a level that meets the Hydrid Electric Vehicle (HEV) goals without compromising power or lifetime. The scientific goal has been to provide understanding of the nanoscale interfacial phenomena that control the device performance and by doing so provide a basis to obtain understanding of interfaces in the supercapacitor area as well as other energy conversion devices (Dye Sensitized Solar Cells, Organic Light Emitting Cells, batteries and fuel cells). In addition to conventional capacitor electrolytes and electrodes we have investigated the performance of ionic liquids to increase safety and redox polymers, conducting titanium oxides and some intercalation-type electrodes that allow us to increase the device capacity. This work seeks to broaden the applicability of skills possessed by the battery group into other areas thereby providing the potential for a wider diversity of support for the electrochemistry group.

\section{Accomplishments}

Following on from year one of the project in which we set up the testing facilities and initiated preliminary experiments on the targeted materials we have successfully completed the examination of all the systems that we targeted except for some ionic liquid systems which proved to be extremely difficult to obtain. Sources of these materials have now been found and experiments on these will be the subject of future funding requests. In addition to the electrochemical testing we have developed new analytical methods for analysis of electrolytes which allows us to track the degradation products and hence relate the observed chemistry to the changes in electrochemical performance. We also developed methods of measurement of the physical properties of the electrolytes (e.g. viscosity) and attempted to establish correlations between the physical properties and the observed electrochemical properties.

We found that carbon remains the best substrate electrode material for capacitor functions due to the ease with which its surface can be optimized (both in area and surface functionality). Samples of conducting Titanium oxides proved to have insufficient surface area for the purpose. To date we find that the carbon does not deteriorate with time or cycling sufficiently to warrant development of a new substrate material. The use of Redox-active polymers to increase the capacity and voltage was partially successful and sufficiently so to warrant further work. This will be the subject of future funding requests. There is a significant stability issue with these systems that must be solved that future work will investigate. The use of these redox-active polymers in capacitors provides useful information that carries over to their use in fuel cells, and electrochemical sensors (e.g. glucose sensors).

The project generated one poster presentation at the October 2010 Electrochemical Society Fall Meeting in Las Vegas, NV and presently there are enough results for at least two full papers. Manuscripts for these papers are in preparation. 


\author{
Probabilistic Optimization of Energy Systems in Buildings \\ Principal Investigators: David Lorenzetti and Michael Sohn
}

\title{
Project Description
}

We will develop an algorithm for optimizing, in a probabilistic sense, the design of building energy systems. Probabilistic designs seek to optimize the expected value of a cost function (for example, the expected energy use), in the face of uncertainty in the variables that affect the problem (for example, uncertain heating loads and equipment failure rates). However, probabilistic design problems lead to a "combinatorial explosion" in both the number of designs that must be considered, and the effort needed to evaluate each design. This may push designers to artificially limit the number and scope of uncertain variables they will consider.

We will explore algorithms that manage this combinatorial complexity by: (1) incorporating expert judgement about likely solutions; (2) identifying dependencies among the design parameters; (3) systematically reducing the design space in light of results from earlier designs; and (4) producing useful partial results even if the algorithm does not run to completion. To do so, we will frame the optimization as a Bayesian updating problem, in which distributions of design parameters are updated to reflect the information gathered by evaluating candidate designs. At any given point in the process, the universe of possible designs will be represented as individual or joint distributions of design parameters. Initially, the distributions will represent expert judgment about the likely values, but as the optimization progresses, the distributions will come to reflect the actual performance of the designs tested so far.

\section{Accomplishments}

Work focused on an extended case study: the design of a duct system for a commercial building. The design goal was to minimize energy use, subject to realistic constraints (e.g., minimum and maximum duct velocities, and maximum duct heights).

To ensure realism, we used an existing model of an actual building. The model was based on drawings and on-site inspections, and calibrated using tracer-gas experiments. The model has a moderately detailed duct system, showing ducts of different cross-section, down to the terminalbox level. A more faithful model would include specific energy losses at duct size transitions, and the flex-duct leading from the terminal boxes.

To define a probabilistic problem, we developed a number of scenarios, representing different demands for fresh air and cooling throughout the building. For example, one scenario has strong afternoon sun heating one face of the building, another has flows dominated by internally-generated heat. These scenarios vary not only in the distribution of air on each floor, but also in the total flow rate of air demanded by each floor of the building.

We weighted each scenario to reflect its likelihood of occurence, then defined a cost function to reflect the weighted energy use for a particular duct design. The cost function also included penalties for violating duct velocity guidelines.

While we are not done analyzing the results of this case study, the most important conclusion is how poorly existing simulation tools support numerical experiments of this kind. Most of our work went into automating the process of modeling a particular duct system, running the tool multiple times in order to tune the control system parameters, and then extracting the information needed to compute our cost function. The tool provided little support for any of these tasks-all of which are important in a probabilistic design context. 
Relating Tissue Residues to Indoor Chemical Sources in a Bayesian Framework:

Synthesis of Chemistry, Pharmacokinetics, and Biomarkers

Principle Investigator(s): Randy Maddalena, Tom McKone, and Michael Sohn

\section{Project Description}

Organic chemical residues from agriculture, industry, building materials and consumerproducts are widely found in the environment and in biota, including human tissues (blood, urine, lipids, etc), which is raising concern about exposures and potential long-term health impacts. Efforts to assess human and ecosystem exposures through multiple environmental media have produced a number of useful modeling and monitoring tools and techniques. Unfortunately the indoor environment has been excluded from much of this work, particularly with respect to persistent and bioaccumulative semi-volatile organic chemicals (SVOCs). As a result, public health agencies continue to assess residential exposures based on sparse concentration data and detailed time-activity models. This approach requires extensive activity data, is awkward, full of assumptions and uncertainty, and difficult to validate or apply to emerging exposure biomarker data, particularly when product and chemical streams are rapidly evolving to meet the increased demand for high performance energy efficient building.

The overall goal of this project is to provide a more systematic understanding of the relationships among indoor contaminants, indoor chemistry, human exposure, and tissue burdens, using a combined modeling and measurement strategy. Our approach is to build on a well-established modeling philosophy that uses physiochemical properties, chemical potential (fugacity) and mass-balance concepts to link the indoor chemical fate processes to biomarkers of exposure.

Accomplishments:

We wrote a detailed research request to the National Center for Health Statistics (NCHS) to gain access to the confidential NHANES 2005 biomarker data. The request was approved so that we can now perform the statistical analysis of the biomark data and associated individual descriptive variables at the NCHS Research data Center (RDC) in Maryland. A manuscript is in preparation that describes the analysis of the NHANES Biomarker data and associated individual and residential characteristics as well as spatial analysis (by urban-rural county). Individual characteristics include age, ethnicity, and poverty/income ratio. Residential characteristics include type of home (mobile home, single-family attached or detached, or other), source of tap water, whether painting conducted in home over past 12 months, presence of attached garage, and whether an insecticide was sprayed in the home over past 12 months. A literature review was completed on measured levels of key indoor semivolatile organic compounds in humans, the use of biomarkers in human exposure assessment, and on the modeling to examine the role of the indoor environment in leading to human exposures and manuscript describing the review is in preparation. We also established several analytical chemistry methods for measuring and quantifying semivolatile organic compounds in the indoor environment that are allowing us to include these measurements in field studies that have previously focused exclusively on volatile organic compounds. 


\section{Self-Tuning Building Energy Model \\ Principle Investigators: Phillip Price, Douglas Black}

\section{Project Description}

The goal of this project was to create a software tool that automatically adjusts, or "tunes," the parameters of a building energy model to match data (such as indoor temperatures, lighting and cooling energy, etc.) from a real building. Such a tool greatly improves the speed with which a building energy model can be created, and results in a model that matches the data better than does a model created by a human who adjusts model parameters.

\section{Accomplishments}

Software, which we call Software for Tuning Building Energy Models (STEM), was written to automatically modify parameters of a building energy model - specifically an Energy Plus model; this is the current state of the art in building energy modeling software - to minimize an error metric that summarizes the discrepancy between model predictions and building data. Both single-processor and parallel-processor versions of the software were created. The software was demonstrated using data from a large academic building that includes both classrooms and offices.

Prior to the creation of STEM, tuning a building energy model for a complicated building could take several months of work by a skilled Energy Plus modeler, even after the basic structure of the model is defined. The resulting model is a single instance of a model that fits the data as well as the modeler was able; there is no assurance that the resulting model could not be greatly improved with a different choice of parameters, nor is there any ability to determine which parameters are or are not tightly constrained by the data. In contrast, with STEM, approximately one to two work-weeks are needed to define the relationship between available building data and the output of the Energy Plus model; the software can then perform the optimization, with some assurance that the resulting model will be among the best-fitting models possible. Additionally, the parallel version of the model provides information about the sensitivity of the model to each of the input parameters, allowing determination of which parameter values can be precisely determined from the building's data, and which cannot.

Funding for further development of STEM has not been identified, but STEM is now being used as part of one LBNL project and is being contemplated for another. The days of laboriously hand-tuning models should be nearly over. 


\title{
Building Systems for Net Zero Energy Buildings
}

\author{
Principal Investigator(s): Michael Wetter (LBNL), Wangda Zuo (LBNL), Alberto \\ Sangiovanni-Vincentelli (UC Berkeley)
}

\section{Project Description}

This project is to prototype how integrated building systems with very low energy use can be designed by using model-based rapid prototyping within a formal design process based on a platform-based design (PBD) methodology. For the rapid prototyping, we will use the Modelica language, which is an equation-based, object-oriented modeling language for dynamical systems. Research challenges include how to scale equation-based models from component level to whole building level for annual energy optimization subject to comfort constraints. To formalize the design methodology, we will prototype the PBD flow for building automation systems. PBD is based on the usage of formal modeling techniques, clearly defined abstraction levels and the separation of concerns to enable an effective design process. Research challenges include how to define abstractions for the variety of building control algorithms and product lines to ensure a robust design flow that reuses system models for energy analysis, design of the building automation system and deployment of control sequences.

\section{Accomplishments}

Our first accomplishment is the selection of building energy systems and control sequences for our design flows, and the creation of component and system models of these systems that allow quantifying energy use, peak power demand and thermal comfort. Compared to leading modeling approaches for building energy systems, ours is novel in the sense that it is declarative and allows a one-to-one mapping between actual control logic and their implementation in simulation, following composition rules that allow a functional decomposition into control systems and energy systems.

Our second accomplishment is the formulation of these models for scaling them to whole building systems. When models of subsystems were combined to whole building systems, the numerical problems were beyond the capabilities of current numerical solvers. By reformulating models and underlying library functions, we were able to simulate nonlinear dynamic models at the whole building level that were composed of thousands of components. We also successfully linked these models to other whole building envelope simulation programs for co-simulation, thereby allowing the reuse of models that would be impractical to rewrite within this project.

Our third accomplishment is the successful prototyping of our PBD flow on a temperature control model originally specified in Simulink. The input specification is translated to an intermediate format, and then automatically mapped onto an implementation platform, including sensors, actuators, embedded processors, and communication network distributed on a floor from a real office building. The mapped design is then exported to the vendor specific language, and compiled to target code. In addition to the code generation for individual processors, the communication interface between the processors is synthesized in order to preserve the behavior of the original functional model. We now work on the development of this flow for the Modelica based specification. 


\section{LB09004}

\section{Genomics Division}

Measurement of Protein/DNA Binding in vitro and in vivo Using Single Molecule Approaches Principle Investigators: Mark Biggin and Jian Jin

Project Description

Correct modeling of the interaction of sequence specific transcription factors with DNA is critical for systems studies of complex transcriptional regulatory networks, such as those controlling animal development. We are developing novel single molecule methods to accurately measure (i) the affinity of factors to a range of binding site variants in vitro and (ii) methods to measure the relative levels of factors binding to defined genomic regions in the embryo with cellular resolution. These methods will provide a much more accurate understanding of transcription factor behavior and improve quantitative modeling.

For the in vitro assay, single protein molecules will be immobilized on within a nano array and the dwell time and intensity of fluorescently labeled DNA oligonucleotides will be used to establish the protein/ligand affinities. A micro fluidic system will be established to allow rapid interactive measurement of multiple DNA binding site variants. The accuracy of the resulting affinity models will be benchmarked against earlier in vitro models using the relative enrichment of sites predicted by each model in genomic regions known to be bound in vivo.

For the in vivo assay, endogenous transcription factors and a specific target cis regulatory DNA region will be labeled with different color nano particles in fixed Drosophila embryos. Using super high resolution light microscopy, the co localization of proteins and DNA will be measured cell by cell within parts of the embryo where factor protein concentrations vary. The relative occupancy of protein on DNA will then be calculated.

Accomplishments feasibility.

We have reached important milestones on both projects, illustrating their technical

In vitro measurements. Before fabricating the nano scale array, we first generated proof-of-principle single-molecule data by immobilizing transcription factor proteins on PEG (polyethylene glycol) passivated glass cover slips. By analyzing the binding/unbinding events, we showed we were able to infer absolute dissociation constants. We have also manufactured an array and established that these bind to transcription factors. A four chambered automated micro fluidic device has also been built that will allow measurements to be made for a whole series of different affinity DNAs with no intervention after initial set up.

In vivo measurements. The major technical obstacles for measuring co localization of transcription factor proteins and DNA are to specifically label each with fluors suitable for single molecule detection and to detect these fluors in the embryo at high resolution. To this end, we have shown that we can use a 3D tracking microscope developed by the Yang laboratory to locate and extract super resolution localization of a single protein molecule labeled with single quantum dots. Since significantly more photons can be detected from single quantum dots compared to that from a single fluorescent dye, we estimate that the resolution is limited by the size of the label. We have also succeeded in labeling a short single copy DNA CRM and obtained excellent images. 


\section{Assessing Epigenomic Approaches for Gene Enhancer Discovery PIs: Len A. Pennacchio, Axel Visel}

\section{PROJECT DESCRIPTION}

Sequencing of the human genome, achieved with substantial contributions from DOE/LBNL/JGI, has provided a near-complete list of human genes. In sharp contrast, these efforts have identified only a marginal fraction of the regulatory sequences that play crucial roles in development and disease as they determine when and where each gene is expressed. Modest recent progress towards this goal has been made through the use of vertebrate comparative genomics. The goal of this LDRD is to generate and explore genome-wide maps of enhancerassociated transcriptional co-activators in embryonic mouse tissues as a strategy to accurately identify tissue-specific enhancers. We will also determine if such epigenetic modifications exist in non-conserved DNA. Technologies developed through these efforts will represent significant achievements with strong impact on the field, as they will greatly increase our ability to identify enhancers and enable exploration of their roles in human biology and disease.

To identify sequences with chromatin modifications, we will use Chromatin-ImmunoPrecipitation coupled to massive-parallel sequencing (ChIP-seq) to capture nuclear DNA sequences with desired chromatin modifications. We will then perform transgenic mouse assays on DNA fragments with desired epigenetic features to assess their predictive power for enhancer activity. These studies will enable genome-wide identification of embryonic enhancers and facilitate their functional characterization in transgenic mice.

\section{ACCOMPLISHMENTS}

Through this LDRD, we established ChIP-seq as a highly efficient method for mapping of chromatin marks in a variety of embryonic and adult mouse tissues. This included the development of laboratory and computational techniques for the generation of ChIP-seq datasets, the evaluation of several potentially relevant marks for this purpose, and the large-scale experimental validation of some of these marks (in particular p300 and CBP) in transgenic mice.

The methods established through this LDRD and the datasets produced were described in two papers, published in the journals Nature and Nature Genetics. The first of the two published studies provided proof of principle for the identification of tissue-specific enhancers by ChIPseq. In the second study, we undertook an in-depth comparison of the evolutionary conservation characteristics of p300 binding sites in different tissues. Additional publications that are based on accomplishments made through this LDRD are in preparation.

The basic method development and the generation of initial datasets enabled through this LDRD were also critical in obtaining external funding. Based on results from year 1 of this LDRD, Dr. Visel obtained a grant from NIDCR (1U01DE020060-01) to apply methods developed through this LDRD to study regulatory mechanisms of craniofacial development and the genetic basis of craniofacial birth defects in humans. In year 2, Dr. Pennacchio obtained an NHGRI grant (R01-HG003988), through which he will apply concepts and methods established in this LDRD project on a larger scale to study occupancy profiles of an entire panel of transcriptional co-activators in multiple tissues in embryonic and adult mice with the goal to provide a comprehensive functional annotation of the enhancer architecture of the human and mouse genome. 
LB10040

\section{Life Sciences Division}

Synchrotron-Based Microtomography for Functional Analysis of Normal Tissue and Tumor Molecular Markers, and their Perturbation by Low-Dose Radiation Exposure

Principal Investigator: Eleanor Blakely

\section{Project Description}

The first goal of this project is to investigate novel nanoscale methods to optimize microtomography to generate a molecularly-defined, cell-type-specific 3D image of a mouse. A second goal is to use these enhanced contrast techniques to identify functional physiological changes in multiple organ systems after exposure to low doses of radiation in intact animals. The third goal is to detect microscopic cancer cells potentially distributed in multiple organs of a tumor-laden mouse in situ. These goals will contribute to discovery science.

We will image rodents with and without an intravenous injection of novel imaging agents to cell-specific structural proteins. The microtomographic imaging will be done with both dual-energy and fluorescent tomography at the Advanced Light Source. The contrast sensitivity of this technique will be much higher than conventional single energy imaging. The protein specific agents will allow specific targeting to cells and will allow us potentially to generate an atlas of normal tissue markers for several strains of mice. We will then use the contrast enhancement techniques developed in our first goal to identify functional physiological changes in multiple organ systems after exposure to low doses of radiation in intact animals, or in tumor-laden animals, in order to detect microscopic disease. In a time course extending up to 10 months after a low-dose radiation exposure, animals will be injected with the imaging agents, and the distribution of organ-specific changes or nascent tumor cells will be tomographically imaged in situ.

\section{Accomplishments}

Our most significant accomplishment is to successfully obtain high quality $3 \mathrm{D}$ full-body images of several mice at beam energies between 22 and $33 \mathrm{keV}$. We were able to clearly visualize the mammary glands with a voxel size of $18 \times 18 \times 18$ microns. We also measured the elemental sensitivity of the dual energy tomography technique at the palladium $\mathrm{K}$ edge using small vials of solution containing known amounts of a contrast agent. We found that the concentration of Pd needed to have good quality images was not currently possible with the imaging agents that we can make. However, using the dual energy mode at the iodine edge and a tail vein injection of Iohexol, an iodine containing contrast agent, we obtained high quality images of the kidneys in a mouse. We also demonstrated that we could use fluorescent tomography to significantly improve the elemental sensitivity. Fluorescent elemental microtomography with special high contrast agents will allow the targeting and imaging of biological processes. The high dynamic range of the images allows visualization of details in tissue density not possible to achieve with conventional imaging systems. 
Linking genomics, proteomics and ultrastructural characterization of microbial communities and their viruses

Principal Investigator: Luis R. Comolli

\section{Project Description}

The goal is to establish and validate capabilities needed to develop a new field defined by the integration of molecular-scale biological (genes and proteins) and ultrastructural information for cultivation-independent analysis of natural microbial communities. Subsequently, we envisage a major initiative in which this approach is applied to consortia of DOE and NIH interest, including communities involved in bioenergy and bioremediation and human health. In a first stage we proposed to use cryoelectron tomographic (cryo-ET) methods to test hypotheses about biofilm establishment, organization, and dynamics that stem from community proteogenomic analyses of a model system: the multispecies chemoautotrophic microbial biofilms from the acid mine drainage (AMD) system within the Richmond Mine, Redding CA. We also proposed the development of a methodology for the identification of all the species in the biofilms by correlated cryo-electron microscopy (cryo-EM) and Fluorescence In-Situ Hybridization (FISH). In future research we will focus on the development of cell-surface specific probes for selected species in the biofilms based on proteomics and genomics data.

Accomplishments

The main scientific accomplishment is the coupling of near complete reconstruction of the genomes for three, deeply-branched, uncultivated lineages of Euryarchaea from AMD communities with 3D cryo-ET reconstruction-based analysis of cell features and community interactions. A number of 3D data sets clearly show penetration of the cell walls and cytoplasmic membranes of nanoarchaea, designated as ARMAN, by single membrane bounded protuberances from cells of the archaeal order Thermoplasmatales. These features, along with many genes previously only affiliated with Crenarchaea or Bacteria, illustrate novel biology in organisms branching near the Crenarchaea-Euryarchaea split.

Due to limitations in current methods, the primary uncertainty in the analysis is the identification of the specific organisms involved in the documented associations. This obstacle motivated the main methodological accomplishment in the work. Specifically, we have developed a protocol for correlative cryo-EM with Fluorescence In-Situ Hybridization (FISH). An article describing this work is currently in the final stages of revisions and will be submitted for publication shortly. We anticipate that the ability to correlate ultrastructural and metagenomic information in intact community samples will be widely applicable and should open the way for ground breaking new insights in microbial ecology and biology. A simple, but far-reaching innovation we have developed is the ability to prepare cryogenic samples for cryo-EM directly on the field. A portable cryo-plunger device was designed and built for this purpose, and successfully tested in three sites: Iron Mountain, Redding, CA; DOE’s Uranium Mill Tailings Site in Rifle, CO; Lake Tyrrell, Sea Lake, Australia. Intact environmental samples are now being imaged routinely. 
Identification of genetic networks controlling susceptibility to radiation-induced carcinogenesis Principle Investigator: Jian-Hua Mao

\section{Project Description}

Biological responses to radiation exposure - DNA damage and tumor development - are controlled by a multiplicity of genetic factors, most of which remain unknown. The overall goal of this project is to exploit the power of mouse genetics, together with novel developments in genomics to identify genetic networks that confer resistance or susceptibility to radiationinduced DNA damage and tumor development. The identification of human homologues of these predisposition genes and discovery of their roles in carcinogenesis will ultimately be important for the development of methods for prediction of risk and prevention for human cancers after relatively high doses of radiation used to treat diseases in the clinic.

We will use a Systems Genetics approach to identify the main features of the genetic architecture of gene expression in the normal thymus, and to characterize the changes in this architecture that take place during perturbation by radiation or tumor induction in mouse interspecies crosses. We will use a genetic approach that exploits the genetic and phenotypic diversity in mouse inter-species crosses, to examine the effects of radiation in different genetic backgrounds. We will characterize gene expression in normal thymus from interspecific backcross animals to identify genetic variants that control gene expression in the normal unperturbed thymus. These gene expression networks will provide the basis for investigation of the changes induced in mouse thymus by treatment with radiation, and of the relationship of these changes to lymphoma susceptibility. These data will be integrated with QTL analysis of susceptibility to radiation-induced genomic instability and lymphoma. In addition to these analyses of the relationship between germline polymorphisms, gene expression, and lymphoma susceptibility, we will identify allele-specific somatic changes in radiation-induced lymphomas from a population of interspecific backcross mice using a combination of array CGH and genotyping. Linkage to specific genetic changes, or preferential gains or losses of parental alleles will identify candidate susceptibility genes. These data will provide a comprehensive picture of both the somatic genetic events and the genetic susceptibility factors that contribute to the genesis of lymphomas induced by radiation.

\section{Accomplishments}

Our most significant accomplishment is that we have obtained 400 thymuses that were collected from a backcross of the (Spretus/Ei x FVB/N) F1 mice with the parental FVB/N strain (F1Bx). Half of them were subjected to a single dose of 4Gy whole body x-radiation. We are in the process of SNP genotyping these mice by using Illumina Mouse LD Linkage panel that contain an optimized of 377 loci in mouse genome, and in the process of making RNA from thymuses for gene expression analysis using Affymetrix platform. We anticipate to generate gene expression networks for the normal unperturbed thymus and to characterize the changes in gene expression networks that take place during perturbation by radiation. In addition, over 100 same F1Bx mice have been subjected to a single dose of 4Gy whole body x-radiation and are being monitored for tumor development. Thymic lymphomas and some other tissues including tail, lung, liver, kidney and spleen, have been collected and stored in tissue bank. Thymic lymphomas will be analyzed by $\mathrm{LOH}, \mathrm{CGH}$, gene expression micorarray. We will continue to generate F1Bx mice until we have a total of $400 \mathrm{~F} 1 \mathrm{Bx}$ mice for radiation-induced tumorigenesis. 


\section{SPARKLE: A Fluroescence Energy Transfer Methodology \\ Principal Investigator: Cynthia T. McMurray}

\section{Project Description}

In light of advances in visualization technology and the wide range of fluorescent probes, it is now possible to place proteins and D NA in 3-dimensional space inside cells, to monitor changes in sub-cellular location of targets with time, and to identify molecules that are close in space. By employing cadmium selenide (CdSe) quantum dots (QDs), we are developing a technology we call SPARKLE to track intracellular features in real-time and applying it to understand Huntington's Disease (HD) at the cellular level. The em ission spectra for QDs are much more tightly defined com pared to conventional dyes or fluorescent proteins, effectively doubling the number of discernable color labels. This will allow us to in sert up to 7 different colors of QD into cells and simultaneously label structures of interest in HD.

We will develop methods for efficiently producing color-tuned QDs and labeling proteins. Additionally, we will use laser-scanning microscopy and realtime fluorescence imaging to track movement of labeled particles in vivo. The data will allow us to quantitate the behavior of multiple players in HD and visualize their interactions.

\section{Accomplishments}

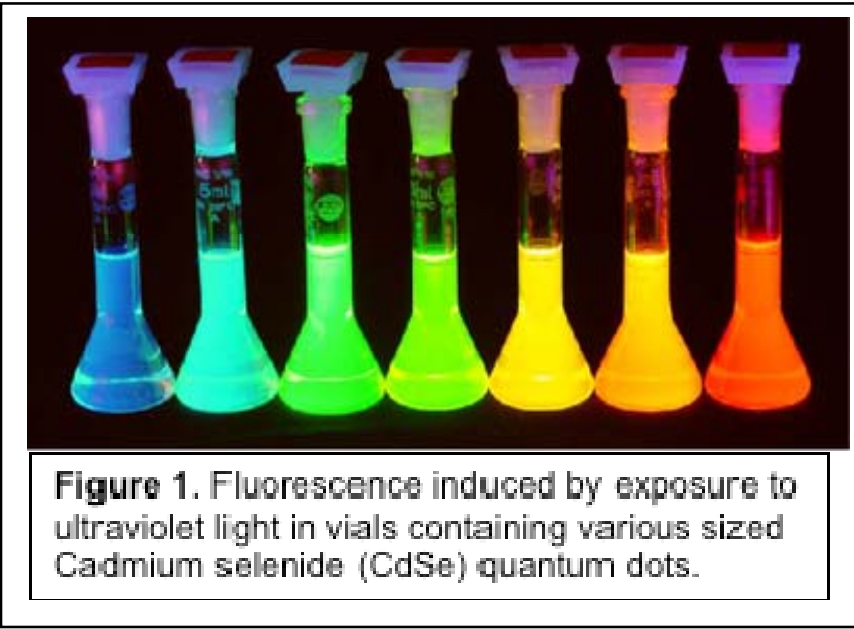

Our FY2010 acquisition of a Zeiss LSM 710 microscope now enables us to spectrally differentiate up to 7 different QD labels and track them in space and time.

The first goal was to prepare QD that were aqueous soluble, non-toxic, stable in solution without aggregation, non-imm unogenic, cell-perm eable. We have developed new methods to optimize production qu ality of QD, which improve homogeneity and usefulness for tissue penetration and cellular uptake in vivo. QDs were prepared in various sizes, suitable for use in the SPARKLE assays. One of the most important advances is the synthetic "normalization" of QD size using WANDA, the Molecular Foundry's one-of-a-kind autom ated parallel synthesis robot. We are now able to synthesize monodisperse QD at large scale.

Additionally, we generated QDs passivated with a well-known non-toxic FDA-approved polymer for in vivo work. In turn, we developed several novel techniques for the chemoselective ligation of the polymer to biomolecules and were successful in our attempts with controlled interactions. The extension of these techniques to link passivated QD to HD protein and other organelles will enable us to track and study their interactions in real time. 


\section{Syngeneic Mouse Model for Breast Cancer Metastasis and Organ Tropism. Principal Investigator: Antoine M. Snijders}

\section{Project Description}

Breast cancer is the second leading cause of cancer death in U.S. women. Metastases are the primary causes of breast cancer related deaths, with death rarely resulting from local disease. Breast cancer metastasizes to local lymph nodes and distant organs including primarily bone, lung and liver and less frequently adrenal glands and brain. The propensity for metastases to specific organs is consistent with the "seed and soil" hypothesis, and differential expression signatures have been developed that predict metastases of ER negative breast tumors to lung. Moreover, greater predictive power was obtained by combining signatures, suggesting that these signatures have only partially captured the complexity of metastasis. We hypothesize that primary tumors acquire genetic and/or epigenetic determinants for metastatic tropism well before they infiltrate local vasculature to metastasize, that integrative expression signatures of the component cells of the tumor tissue will predict general risk for metastasis as well as proclivity for specific metastatic organ sites. To begin to test this hypothesis we propose to develop a syngeneic mouse model system for selection and characterization of specific organ tropisms within mammary tumor cells and associated stromal cells using well-defined genetic models of mammary cancer. It is well known that the tumor microenvironment plays a major role in cancer progression, their role in the acquisition of metastatic signatures in breast cancer remains unclear. Cancer associated fibroblasts (CAF), a major component of the tumor microenvironment, can influence tumor progression and metastasis by producing growth-factors and cytokines that enhance tumor growth and by remodeling the ECM to create an environment permissive for tumor cell invasion. We hypothesize that signatures of metastatic potential in primary tumors are determined by the signaling between tumor cells and CAFs and controlled by both genetic and epigenetic mechanisms.

\section{Accomplishments}

Our initial work on this project focused on understanding the contribution of CAFs towards the primary tumor metastatic signature. We isolated mammary fibroblasts of several inbred strains of mice that differ in their susceptibility to mammary cancer (BALB/c and $\mathrm{C} 3 \mathrm{H}$ mice are sensitive to breast cancer while SPRETUS/EiJ and C57B1/6 inbred mice are resistant). We treated independent derivations of fibroblasts to TGFbeta, a major inducer of the CAF phenotype and characterized the fibroblast secretome using conditioned media. We observed differences in the secretome of mammary fibroblasts isolated from mammary gland cancer sensitive and resistant strains of mice. We are in the process of investigating how CAF secreted proteins determine tumor cell growth and invasion using in vitro culture systems. Mammary tumor cells are cultured in 3D spheroids in a collagenous matrix in the presence or absence of these proteins. Invasion of tumor cells into their microenviroment will be quantified by the dysmorphology of the spheroid outgrowths and the length of cell-tracks into the surrounding matrix.

Once we understand the contribution of genetically defined CAFs to the metastatic signature of breast tumors, we will initiate experiments to dissect their relative contributions to metastatic signatures that predict organ proclivity for specific metastatic organ sites by using an orthotopic transplantation of tumor cells into the mammary fat pad to establish metastatic cell lines with organ site specific tropism 
Bio Energy Technologies and Science Integrated Efficiently (BETSIE) Principal Investigator: John A. Tainer

\section{Project Description}

The growing energy crisis is conceivably the single biggest problem facing our society today and will certainly remain such over the next few decades. BETSIE seeks to exploit naturally occurring bio-systems for efficient carbon cycling and "photons-to-biofuels" conversions. Despite the enormous promise of biology to provide efficient energy conversion technologies, these systems have not been understood in sufficient detail at the molecular level to usefully harness their potential. BETSIE has three fundamental working hypotheses. First, we propose that energy conversion processes are governed by integrated pathways chosen, controlled, and coordinated by the same types of molecular controls that we have helped discover and characterize the molecular interactions and assemblies that protect the cell from cancer. These controls include dynamic macromolecular assemblies, handoffs, conformational switches, and disorder-to-order transitions. Second, we hypothesize that a complete knowledge of these pathways, interactions, assemblies, and conformations will allow us to re-design these pathways in predetermined ways with advantages for our energy needs. Third, we hypothesize that characterizations of these pathways as tested by redesign experiments will allow us to transplant functional pathways into organisms as needed for efficient energy production. We aim to use biochemical and structural characterizations to understand the molecular interactions and controls that are critical for carbon cycling and photon-to-fuel conversions.

\section{Accomplishments}

During FY10, we successfully characterized a key enzyme in the Wood/Ljungdahl $\mathrm{CO}_{2}$ fixation pathway by small-angle X-ray scattering (SAXS), which was Goal 1 of the previous funding period. Using Acetyl-CoA synthase (ACS) from Pyrococcus furiosis, we observed that the solution structure of the ACS alpha subunit did not match the scattering profile calculated from the crystal structure, which suggested that the ACS alpha subunit is a dimer (Fig. 1). Rather, the SAXS curve suggests that the ACS alpha is a trimer in solution (Fig. 1). These results are important not only for understanding carbon fixation, but also for understanding the $P$. furiosis glycolysis pathway that is important for hydrogen production.

We have also conducted fractionation experiments on the green algae Chlamydomonas reinhardtii to isolate native protein complexes important in the regulation of chlorophyll antenna size in chloroplasts. Characterization of the molecular machinery responsible for chlorophyll antenna size is of interest to algal biotechnology and biofuel production since mutants in this pathway would have greater light penetrance and enhanced productivity in high-density cultures.
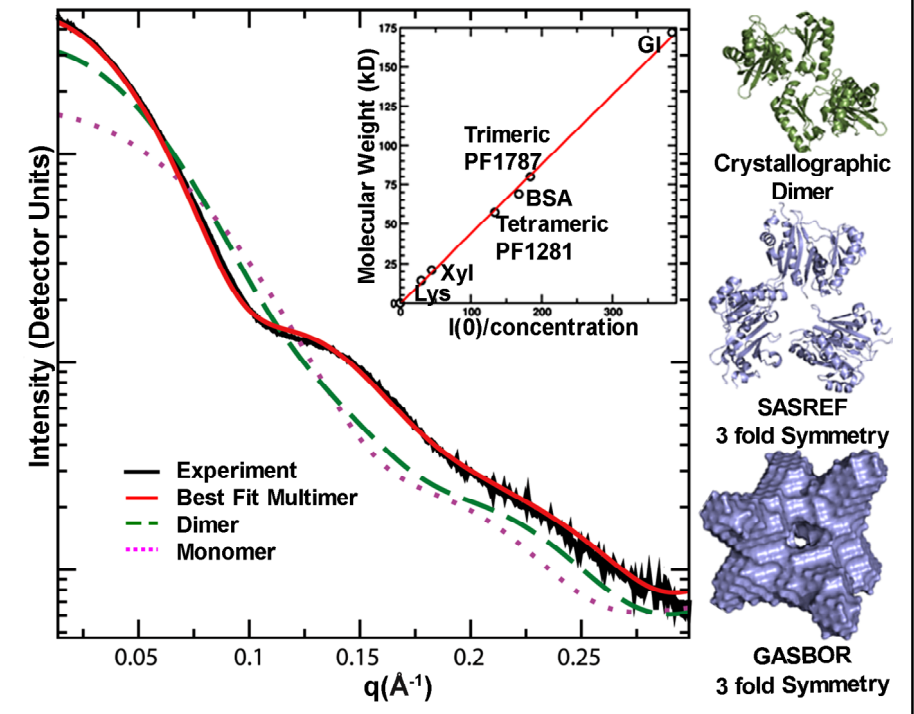

Fig. 1. Characterization of ACS alpha subunit from $P$. furiosis by SAXS. Solution scattering suggests that ACS is a trimer in solution rather than the dimer suggested by crystallography. 


\section{Multinozzle Arrays for Single Cell Metabolomics Principal Investigator(s): Daojing Wang}

\section{Project Description}

The purpose of this project is to develop breakthrough multinozzle array-based electrospray mass spectrometry for single cell metabolomics. Cellular heterogeneity arising from stochastic expression of genes, proteins, and metabolites is a fundamental principle of cell biology, but single cell analysis has been beyond the capabilities of 'Omics' technologies. This is rapidly changing with the recent examples of single cell genomics, transcriptomics, proteomics, and metabolomics. The rate of change is expected to accelerate owing to emerging technologies that range from micro/nanofluidics to microfabricated interfaces for mass spectrometry to third- and fourth-generation automated DNA sequencers. Single cell analysis is the new frontier in Omics, and single cell Omics has the potential to transform systems biology through new discoveries derived from cellular heterogeneity.

However, single cell Omics presents unprecedented challenges. There is a great need for further improvement in miniaturization, integration, and detection sensitivity. There is also a great need for automation, throughput, and bioinformatics to study multiple individual cells to achieve statistical significance.

We propose to develop silicon-based multinozzle arrays and demonstrate their proof-ofprinciple applications of in single cell metabolomics. This work is built upon our recent exciting development of silicon/silica-based microfabricated monolithic multinozzle emitters $\left(\mathrm{M}^{3}\right.$ emitters) for mass spectrometry. We will utilize human mesenchymal stem cell (hMSC) as the initial biological system for testing the new devices since these cells are critically important for regenerative medicine and the PI has extensive working experience with them. Specifically, we will fabricate prototype multinozzle arrays, measure their physical characteristics, perform surface derivatization, and demonstrate their proof-of-principle applications in metabolomics for human mesenchymal stem cells.

\section{Accomplishments}

We have made important progress for the project. Our most significant accomplishment has been to develop high-density multinozzle arrays and demonstrate their applications in mass spectrometry. We first implemented a modeling approach and achieved the rational design of the multinozzle arrays. This allowed us to achieve electrospray ionization mass spectrometry using the existing mass spectrometer with the voltage supply available. We improved the silicon fabrication processes and achieved reproducible and high-quality multinozzle arrays. We are currently implementing the similar strategy for fabricating ultrahigh-throughput devices. Further optimization of the nozzle dimension, geometry, and emitter material is currently underway.

We have published a paper "Single Cell Analysis: the New Frontier in 'Omics"', Wang D.* and Bodovitz S. (2010) as the cover review for the June 2010 issue of Trends in Biotechnology. We comprehensively and critically reviewed the past, present, and future of the emerging field "Single cell Omics". We have brought on board a postdoctoral fellow with a strong micro/nanofabrication background to continue developing the proposed novel devices.

We have also installed the software and hardware for an imaging system for the electrospray ionization (ESI) mass spectrometry. This allows us to monitor the ESI process for the multinozzle arrays as proposed in the project. We are in the process of further evaluating and testing the new multinozzle array devices. 


\author{
Functional Characterization of NUCKS - a Potential Cancer Susceptibility Locus \\ Required for Recombination \\ Principal Investigator: Claudia Wiese
}

\title{
Project Description
}

The maintenance of genetic stability relies heavily on the high fidelity of homologous recombinational DNA repair (HR). Cells with mutations in HR genes (e.g. BRCAI, BRCA2, PALB2, RAD51 C, and RAD51API) exhibit increased levels of genetic instability, and individuals with such mutations are prone to elevated frequencies of many human cancers. Notably, individuals carrying mutations in one allele of either BRCAI or $B R C A 2$ have a lifetime risk of up to $80 \%$ for developing breast cancer and also display an increased risk for ovarian cancer (- $40 \%$ and $-20 \%$ for $B R C A I$ and $B R C A 2$ mutation carriers, respectively), and mutations in both $P A L B 2$ and $R A D 51 \mathrm{C}$ have also been associated with enhanced breast cancer predisposition. Furthermore, it recently has become clear that negative regulators of HR proteins frequently are up-regulated in sporadic forms of breast cancer that are lacking the classical HR gene mutations.

NUCKS (nuclear ubiquitous casein and cyclin-dependent kinases substrate) is a $27 \mathrm{kDa}$ chromosomal protein of unknown function. NUCKS was discovered in 1985 as a perchloric acid- soluble high-mobilitygroup (HMG)-like protein present in human cells. NUCKS is widespread in vertebrates, abundant in rapidly growing cells and over-expressed in a variety of cancers. NUCKS is located on human chromosome 1q32.1, a genomic region that is commonly gained in breast cancer and is over-expressed in sporadic breast cancer tissues with amplifications relative to tumors diploid for NUCKS.

Intrigued by the fact that NUCKS may share structural relatedness with RAD5IAP1, a protein that is essential for DNA repair by HR, we proposed to test directly the possible involvement of NUCKS in the HR pathway.

Accomplishments
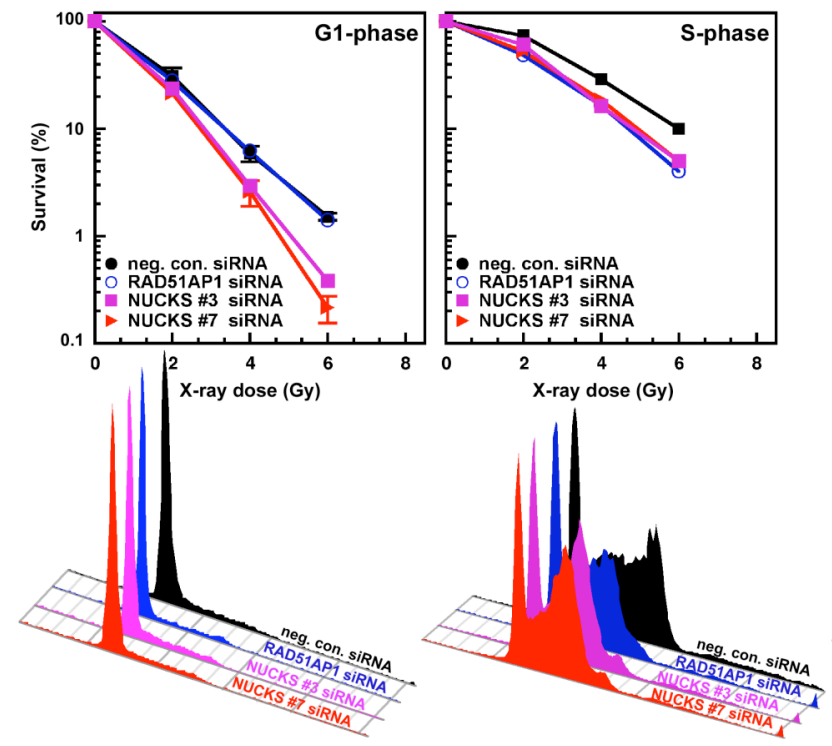

Fig. 1: NUCKS-depleted cells are sensitive to X-rays in G1(left panels) and in S-phase cells (right panels). Upper panels: Cell survival curves from colony formation assays after. Lower panels: Representative cell cycle profiles after synchronization.
Our most significant accomplishments show that - very similar to RAD51AP1 - wild type levels of NUCKS are required for proper HR in human cells. Our results show that knockdown of either NUCKS or RAD51AP1 diminishes the levels of gene conversion (i.e. HR) to about $30-40 \%$ of wild type levels. However, unlike RAD51 AP1 and unlike many other proteins in HR, NUCKS also is required for the repair of radiation damage in G1-phase cells, which - due to the absence of the sister chromatid in G1 - do not perform HR, but instead utilize non-homologous end-joining (NHEJ) for DNA lesion repair (Fig. 1). We conclude that NUCKS is involved in at least 2 different DNA repair pathways and are in the process of investigating this phenomenon that has been described for a select few other proteins as well. 


\author{
Project Title: Self-Assembled Battery Electrodes \\ PI: Nitash Balsara \\ Co-PIs: Andy Minor, Venkat Srinivasan
}

\title{
Project Description
}

Our goal is to create new methodologies and materials for addressing the limitations of current batteries. While current batteries are limited in energy content to about $200 \mathrm{Wh} / \mathrm{kg}$, there is no intrinsic limit on the energy density of a battery. For example, batteries with sulfur cathodes are predicted to have energy densities of $2500 \mathrm{Wh} / \mathrm{kg}$. Similarly, the power limitations of a battery depend on the efficacy of the pathways for ionic and electronic conduction within the battery. We propose to synthesize and study model battery electrodes wherein the pathways for ionic and electronic conduction are controlled on the nanometer length scale. The proposed research will provide fundamental insights needed to design the next generation of batteries.

Functioning battery electrodes require the conducting pathways for both ions and electrons. In conventional lithium batteries, the ions are transported by a liquid electrolyte and the electrons are transported by carbon. Our objective is to synthesize a single polymer material that exhibits both ionic and electronic conductivity. Separate pathways for conducing ions and electrons would be created by the self-assembly of block copolymers. Obtaining such polymers is the first step toward the creation of self-assembled electrodes.

\section{Accomplishments}

The main accomplishment has been the successful synthesis of a diblock copolymer with one ion-containing block and one electron-conducting block. We have thus far synthesized two types of polymers wherein polyethylene oxide (PEO) ion-conducting block: (1) wherein the electronic conductor is poly(pheneylene vinylene) (PPV), and (2) wherin the electronic conductor is is head-to-tail poly(3-hexlythiophene) (PHT). We have quantified the electronic and ionic conductivity of these materials. The polymers have also been combined with conventional battery cathodes such as $\mathrm{LiFePO} 4$ and battery cycling tests with these polymers are currently underway.

We have obtained follow-up funding for the project from the BATT Program. 


\title{
Heating rates of planar ion traps for quantum information
}

\author{
Principle Investigators: John Clarke and Hartmut Häffner \\ March 11, 2011
}

\section{Project description}

The main goals of this project are (a) to study the source of fluctuating patch charges and (b) to device methods to build small traps which feature heating rates on the order of 0.1 quanta/ms or less at room temperature. So far systematic studies of ion heating have concentrated on traps at cryogenic temperatures demonstrating that heating can be suppressed by many orders of magnitude by cooling the electrodes. Here, we will concentrate solely on fabrication methods and materials to create traps which work satisfactory at room temperature. Successful operation at room temperature will facilitate ion trap experiments considerably and allow for many applications ranging from ion trap quantum computing with segmented planar ion traps, extremely sensitive quantum detectors, and interfacing trapped ions with solid state systems. Understanding how to reduce these fluctuating patch charges could also impact other fields like nanomechanics and single spin detection.

We will achieve progress towards these goals by fabricating planar ion traps with different methods. Planar traps have the advantage that they can be produced fast and are costeffective. Furthermore, planar traps have been proven to be easily taken into operation. Finally, the monolithic design of planar traps helps a lot to speed up turn-around times. Traditionally, gold has been employed as an electrode material.

\section{Accomplishments}

We set up a testing apparatus and all the laser sources necessary for loading ions. We trapped ions at Berkeley using a trap fabricated at Zürich. However, the performance was orders of magnitude worse than observed by us previously. This lead us to the conclusion that the trap performance is limited by surface deterioration even inside ultra-high vacuum (UHV), shifting our emphasis to establish surface analysis tools. Currently, we are aiming at investigating the surface of our trap with Auger-spectroscopy as well as with ellipsometry. For this purpose, we build a glove box and initiated a collaboration with the Crommie group at UC Berkeley/LBL.

We also started to establish fabrication of traps using photo-lithography. We successfully patterned the substrate with photo-resist and evaporated a metal onto it. To clean the traps, we build an annealing oven. Along similar lines, we evaluated cleaning procedures inside the UHV chamber. This includes plasma cleaning, photo-desorption as well as heating the trap in situ. Finally, we planned a new UHV trap testing set-up which will allow us fast turn around times. 
Mixed Ionic and Electronic Transport in Solution-Processed Inorganic Nano-Composites Principal Investigator: Delia Milliron

Project Description

This project aims to develop a generalized methodology for deposition of inorganic nanocomposites with controlled composition, morphology, and phase of the component materials. The focus is on demonstrating control over mixed transport properties by independent selection of the matrix material and the nanoparticle inclusions. The resulting complex functionality will enable optimized electrolyte switching, electrochromic, photovoltaic, and thermoelectric materials, for example.

Composites will be prepared from separately synthesized colloidal nanoparticles and molecular precursors, each processed individually from solution, then thermally converting the precursor to form the matrix material. To maintain the integrity of the nanocrystals, precursors will be synthesized which decompose at low temperature. The modularity of the proposed process will be leveraged to vary the composition of each component in order to independently tune transport characteristics such as ionic and electronic conductivity.

\section{Accomplishments}

A modular method for fabricating inorganic nanocomposites from colloidal nanoparticles and soluble chalcogenidometallate clusters (ChaMs) has been demonstrated. Our fabrication process forms superlattice thin films wherein the assembly is mediated by ligands. Next, these ligands are displaced, which are finally decomposed at low temperature to form a continuous inorganic matrix phase in the intra-nanoparticle spaces. The regular ordering of the initial nanoparticle assembly, is largely maintained through the process. This process permits exceptional composition and morphological tunability: we have prepared over 30 different nanocomposites.

In order to systematically examine mixed ionic and electronic transport, we have focused on nanocomposites containing nanocrystals in an amorphous matrix. Because the composites are thermally stable, the phase transition into the high temperature superionic phase, which occurs around $170^{\circ} \mathrm{C}$, can be studied without inducing nanocrystal sintering.

In order to study the mixed transport properties, we have carried out impedance spectroscopic analysis on our composite thin films over a broad frequency range and as a function of temperature. Ionic and electronic transport give rise to distinct frequency-dependent features of the complex impedance spectrum, allowing their respective contributions to the overall conductivity to be evaluated. The thermal activation energy for the overall conductivity of the nanocomposites below the superionic transition is very close to that of $\mathrm{GeS}_{2}$ alone, revealing the dominant role played by the matrix phase in this regime. Above the transition, the activation energy is intermediate between that for $\mathrm{GeS}_{2}$ and that for $\mathrm{Ag}_{2} \mathrm{~S}$, revealing a more balanced role for the two components. Furthermore, the ionic transference number, which is defined as the fraction of the overall conductivity which is ionic (rather than electronic) in nature trends systematically with the $\mathrm{GeS}_{2}$ content of the nanocomposites. Specifically, films containing smaller $\mathrm{Ag}_{2} \mathrm{~S}$ nanocrystals (and therefore a larger volume fraction of the poor electronic conductor $\mathrm{GeS}_{2}$ ), have a higher ionic transference number. These results demonstrate that through our nanocomposite fabrication process we can effectively manipulate transport properties through precise control of morphology and composition. 


\title{
In situ Electromechanical Probing in a TEM
}

\author{
PI: Andrew M. Minor
}

\section{Project Description:}

The goal of this project was to establish the technical ability to probe both the electrical and mechanical properties of individual nanostructures simultaneously, quantitatively and at high resolution in a Transmission Electron Microscope (TEM). This capability enables the study of coupled electrical and mechanical phenomena for a wide variety of materials science applications, including energy scavenging, microstructural evolution in piezoresistive materials, phase transformations during mechanical deformation and exploratory work on individual nanostructures.

The research and development efforts were centered on the design and implementation of a custom-built holder for a transmission electron microscope. The holder has the sensitivity and stability for in situ TEM electromechanical probing of individual nanostructures such as piezoresistive thin films or nanowires. To date, one of the main limitations of probing experiments inside a TEM have come from control of the testing apparatus and the noise floor for quantitative force data. The stability and control of quantitative mechanical testing has come a long way in the past few years, and new control electronics just become available that allow for improved displacement control and feedback in order to decrease the current amount of vibration in the indenter tip. Accurate displacement control is important for in situ small-scale mechanical testing where one would like to anticipate contact and deformation in terms of a spatial constraint (the field of view during the test).

\section{Achievements:}

We used the unique electromechanical in situ TEM holder to investigate the phase transformation behavior of a piezoelectric thin film, a result that was recently accepted for publication in Nature Nanotechnology. Piezoelectrics exhibit a mechanical response to electrical input, or vice versa, that makes them useful in sensors and actuators. This also made them an ideal test sample to demonstrate the capabilities of the in situ electromechanical holder. The ferroelectric $\mathrm{BiFeO}_{3}$ is an attractive alternative to lead-based piezoelectric materials because strain can stabilize $\mathrm{BiFeO}_{3}$ phases with a morphotropic phase boundary-like structure. Our study demonstrated a reversible electric-field-induced strain of over $5 \%$ in the $\mathrm{BiFeO}_{3}$ films, along with a characterization of the origins of this effect. In-situ transmission electron microscopy coupled with nanoscale electrical and mechanical probing showed that large strains result from moving the boundaries between the tetragonal- and rhombohedral-like phases, changing the phase stability of the mixture. These results demonstrated the potential of $\mathrm{BiFeO}_{3}$ as a substitute for lead-based materials in future piezoelectric applications. 


\title{
X-ray Studies of Charge-Order Dynamics in Complex Materials
}

\author{
Principal Investigator: Robert W. Schoenlein
}

\section{Project Description}

The purpose of this project is to develop novel ultrafast resonant $\mathrm{x}$-ray scattering techniques, and apply them to study charge and orbital ordering dynamics in complex materials where strong interplay between charge, spin, and subtle lattice distortions give rise to rich phase diagrams. This program will focus on transition-metal oxides, and their dynamic response to tailored vibrational and electronic excitations. Resonant $\mathrm{x}$ ray scattering (RXS) is a powerful quantitative probe of charge and orbital ordering in complex materials. However, studies to date have focused on ordering phenomena in the 'quasi-static' regime, as a function of adiabatic changes in doping, pressure, temperature, and applied field. Understanding the dynamics of such ordering phenomena, their time scales, correlation lengths, and coupling to different degrees of freedom, is essential for revealing the driving force behind the intriguing physics and novel properties of correlated materials.

Resonant $\mathrm{x}$-ray diffraction measurements are made on Bragg reflections that are nominally forbidden by the lattice unit-cell symmetry. Anisotropy of the electron density (e.g. from charge/orbital ordering) results in non-zero Bragg reflections. These reflections are particularly enhanced at absorption resonances (e.g. L-edge or K-edge) since they directly probe the valence states most affected by the symmetry-breaking. Time-resolved resonant x-ray diffraction experiments will be conducted at ALS BL6.0.1 and BL6.0.2, the only beamlines in the U.S. providing the required tunable femtosecond $\mathrm{x}$-ray pulses in the $0.2-10 \mathrm{keV}$ range, accompanied by a laser system covering the visible and mid-IR range (for tailored excitation). For these studies, a dedicated vacuum chamber and diffractometer will be constructed that will incorporate a cryostat for low-temperature measurements.

\section{Accomplishments}

We have carried out static resonant soft x-ray scattering experiments at ALS BL 8 to characterize the orbital order of our samples, and determined the critical experimental parameters (geometry, photon energy, scattering angle, and sample temperature) to observe the orbital-order peak. We found that the orbital order scattering spectrum in PCMO differs markedly from the measured x-ray absorption spectrum. This indicates that detailed information on the ordered charges may be available from this technique. As a result of this work, we are establishing a new collaboration with a theory group to provide a full interpretation of these results.

A second accomplishment is the demonstration of nanosecond time-resolve resonant softx-ray scattering at ALS BL 6. These studies demonstrate the feasibility of this approach for following the dynamics of orbital ordering. The initial measurements provide particular information on how the ordering develops from a disordered state.

BES.

We have applied for, and received, direct follow-on funding for this research from DOE 
Tuning the self-assembly of membrane proteins

Principal Investigator(s): Berend Smit

\section{Project Description}

Our understanding of the role of the cell membrane has evolved from a passive bilayer limiting the boundaries of a cell to an integral part of the regulatory system of the cell. Linked to this regulatory system are the mechanisms the cell membrane is using to modulate its structure. In this model, the cell membrane contains different morphologically distinct regions or domains specialized in different functions ranging from nutrient adsorption, cell-to-cell communications, or endocytosis. In this proposal we address the question of how cells modulate the structure of membrane proteins and the self-assembly of membrane proteins. In this project we use coarsegrained molecular simulation to study the effect of changes in the structure of the bilayers on the interactions between objects embedded in the membrane.

\section{Accomplishments}

Our most significant accomplishment has been to develop a model for cholesterol and to develop a computational method to compute the solubility of alcohol molecules.

We derived a new density-biased Monte Carlo technique, which preserves detailed balance and improves the convergence of grand-canonical simulations of a species with a strong preference for an interfacial region as compared to the bulk. This density-biasing technique is applied to the solubility of "alcohol" molecules in a mesoscopic model of the lipid bilayer, a system which has anesthetic implications but is poorly understood.

We also developed a coarse-grained model of a hydrated saturated phospholipid bilayer containing cholesterol that we study using a hybrid dissipative particle dynamics-Monte Carlo method. We studied the structural and mechanical properties of the bilayer at various temperatures and cholesterol concentrations. The properties studied are the area per lipid, condensation, bilayer thickness, tail order parameters, bending modulus, and area compressibility. Our model quantitatively reproduces most of the experimental effects of cholesterol on these properties and reproduces the main features of the experimental phase and structure diagrams. We also presented all-atom simulation results of the system and use these results to further validate the structure of our coarse-grained bilayer. On the basis of the changes in structural properties, we proposed a temperature-composition structure diagram, which we compare with the experimental phase and structure diagrams. 
Structured Charged Polymers

Principal Investigator: Matthew Tirrell

\section{Project Description}

The purpose of this project is to characterize and quantify attractive interactions among charged polymers. Highly charged polymers, natural or synthetic polyelectrolytes, and their assemblies, possess unique capabilities for structure determination and manipulation. Biology has created an astonishing array of self-assembled, functional molecular systems that can perform tasks with a precision often surpassing modern manmade systems. Electrostatic interactions are among the very most important non-covalent interactions employed in nature. They are very responsive to easily deployable signals (salt, $\mathrm{pH}$ ). The objective of this project is to create model systems that will enable the development of a fundamental understanding of the factors that govern self-assembly of ion-containing, multi-component polymers, and that produce a range of new and useful properties. Lack of fundamental and quantitative understanding of polyelectrolytes has retarded the exploitation in synthetic materials of their many significant properties, especially in comparison to the ubiquitous role of electrostatics in biological structures. Our emphasis has been on direct measurement of attractions between negatively charged polyelectrolyte chains arising from interactions with multi-valent cations (positive ions from metal salts, such as aluminum, $\mathrm{Al}^{3+}$, lanthanum, $\mathrm{La}^{3+}$, and other tri-valent cations). Attractive interactions permit intermolecular bonding among charged macromolecules leading to possibilities for materials synthesis. Energy implications for polyelectrolyte complexes include use as encapsulants for particles in low power, e-ink displays.

\section{Accomplishments}

The experimental system we have concentrated on is polystyrene sulfonate (PSS) in the presence of various tri-valent cations. The PSS is end-tethered in the form of a polymer "brush" on to a mica or gold surface that has been covered by a hydrophobic monolayer. The brush is formed from block copolymers of PSS with a short hydrophobic anchor block. The anchor block sticks spontaneously to the hydrophobic surface producing a dense layer of end-tethered PSS chains. The hydrophobic mica surface is used in our surface force measurement apparatus (SFA). Two PSS layers are brought together in the SFA in the presence of various ratios (R) of tri- to mono-valent (sodium, $\mathrm{Na}^{+}$) ions, at overall fixed ionic strengths (I). The general observation, made with various tri-valent ions, is that at a well-defined $\mathrm{R}$, the brushes shrink in size due to attractive intra-brush intersections, and, become strongly adhesive to one another, due to attractive inter-brush interactions. $R$ increases with increasing I. These data enable us to quantify the ionic conditions necessary to cause two polymer chains with the same sign of charge can be bound together by multi-valent ions. The hydrophobic gold surface is used in an electrochemical apparatus known as cyclic voltametry. By varying the voltage, one can induce the oxidation (or reduction) of di-valent to tri-valent ions (or the reverse) and, by measuring the resulting current, "count" the number of electrons, and therefore, ions, involved in the process. We see quantitatively that tri-valent ions are more loosely bound in the brush layer at lower values of $\mathrm{R}$ and at higher values of $\mathrm{I}$. We are extending this work to interactions between PSS and soluble cationic polyelectrolytes, for further insight into new materials synthesis via electrostatic forces. 


\section{Understanding How Nanoscale Interfaces Modify Predicted Optical, Vibrational, and Electronic Properties Principal Investigator: Jeffrey J. Urban}

\section{Project Description}

Predicting and measuring the electronic energy levels, gaps, and alignments in nanoscale systems as done for bulk materials, is currently impossible. Moreover, remarkable modifications to bulk optical, vibrational, and structural properties have been reported, however, predictive models and true physical understanding of these phenomena remain elusive. This LDRD has focused on detailed characterization of complex heterostructured nanomaterials to address these issues.

Precise engineering of the generation, flow, and storage of energy in single-phase bulk materials is a mature discipline. Decades of coordinated efforts between experiment and theory now enables the accurate estimation of band alignments at heterointerfaces, carrier transport, and basic physical constants in bulk solids, relevant for device engineering and design. Nanoscale solids are a promising avenue to enhance energy conversion efficiencies and storage densities beyond those of known bulk solids. However, there is no corresponding set of general principles to guide material and device design in these nanoscale systems. Highly inadequate physical models for bulk heterostructured interfaces often constitute the basic intuition for material design. Despite the promise of new emergent phenomena unique to the nanoscale, ignorance of the fundamental rules governing electronic energy level alignment at interfaces must be overcome if new, highperformance technologies are to be realized. Addressing this core scientific problem will require notable advances in the areas of nanostructure fabrication, optical and scanned probe microscopy, and atomistic-level theory.

\section{Accomplishments}

The main accomplishment of this project is the development, implementation, and demonstration of novel nanoscale optical imaging tools that enable optical interrogation of electronic and vibrational properties with unprecedented spatial resolution. In order to examine true nanoscale heterojunction energetics, a new type of optical probe was required. Thus, we have developed and implemented nm-scale plasmonic atomic force microscope tips capable of both enhancing the local optical field several orders of magnitude, as well as allowing transmission through a nanoscopic aperture, thereby producing a sub 15-nm light source. To date we have demonstrated proof of concept, however, in the near future we will use these techniques in conjunction with novel nanoheterojunction samples of appropriate length to resolve important optical and electronic effects $(\sim 4 \times 300 \mathrm{~nm})$. We are uniquely well positioned to make the first measurements of nano-scale charge transfer in materials with sub-Bohr Exciton radius resolution.

In addition, using confocal spectro-microscopy we have shown that it is possible to determine crystallographic orientation of gallium nitride nanowires with diameters below the diffraction limit of light. Also, we have demonstrated that white light can be non-linearly generated in these same wires and that the cavity modes observed within reveal rich detail of the fundamental optical and physical properties of the wires themselves. Both of these observations are due to nanoscale interactions unique to these wires and have revealed the role of nano-interfaces on semiconductor photophysics. 
Project Description:

Graphene, a single layer of honeycomb carbon lattice, is emerging as an exceptional new material for future electronic technology. One reason for this is that graphene exhibits many novel behaviors. Electrons in graphene, for example, move as if they have zero mass and display strongly quantum mechanical behavior even at room temperature.

In contrast to the great activity in graphene electronics, studies on graphene optoelectronic properties have been few. However, our recent studies show that graphene optoelectronics can provide exciting opportunities for physics and technology. For instance, we demonstrated that graphene couples to light strongly and the infrared optical transitions in graphene monolayers can be switched on and off by electrical gating. We have also shown that a continuously tunable electronic bandgap up to $200 \mathrm{meV}$ can be induced through electrical gating in graphene bilayer. These findings open the door for coupled optical and electrical excitations in engineered graphene. Understanding of such optoelectronic reponses can lead to novel graphene based optoelectronic devices.

\section{Accomplishments:}

We have made exciting progresses in studies of tunable electro-optical behavior in graphene in the LDRD project. In particular we performed the first measurement on Drude conductivity of Dirac electrons in graphene. High frequency conductivity of Dirac fermions is a valuable probe of the dynamic electrical reponses of Dirac fermions. Using infrared spectroscopy and large-area graphene samples, we were able to measure the graphene high-frequency conductivity from the terahertz to the mid-infrared spectral range at varied electron and hole concentrations. We show that graphene conductivity exhibits a Drude-like frequency dependence, which becomes extremely strong at longer wavelengths. In particular, at $\mathrm{THz}$ wavelengths $(\lambda \sim 300 \mu \mathrm{m})$ a gated graphene can absorb almost $20 \%$ of the radiation, suggesting that graphene can be an interesting $\mathrm{THz}$ material. More importantly, we were able to determine independently for the first time the Drude weight and electron scattering rate, the two most important parameters in electrical transport. It reveals an anomalous reduction of the Drude weight compared to the widely accepted theoretical predictions. The work has been submitted for publication. 


\section{Design and Surface Properties of Semiconductor Nanowires \\ Principal Investigator(s): Peidong Yang, Ali Javey, Junqiao Wu}

\section{Project Description:}

This collaborate effort seeks to explore the controlled fabrication/synthesis and chemical doping of nanostructured materials, including oxides and III-V compound semiconductors. Our goal is to discover, understand, control, and exploit the functionalities of individual phase boundaries in these nanowires. The experiments are designed to address the following outstanding questions: (1). Is there any fundamental difference between doping in nanowires and doping in bulk semiconductor (single crystal and thin films)? (2). How to quantitatively evaluate the dopant environment, uniformity and induced lattice modification, and consequently any electrical/optical property modification? (3). For surface functionalized nanowires, what are the effects on surface states, wire conductivity, carrier mobility and other optoelectronic properties?

\section{Accomplishments:}

The Yang group; P-type doing in $\mathrm{ZnO}$ has been highly elusive, and yet it is extremely important for the realization of $\mathrm{ZnO}$ based active optoelectronic devices. Due to large self-compensation effect in this materials system, many of early attempts in p-type doping have not been very successful and reproducible. We have systematically investigated the various doping precursors $\left(\mathrm{Li}_{3} \mathrm{~N}\right.$ and $\left.\mathrm{NaCl}\right)$ and used FET and thermopower measurements to characterize the doped $\mathrm{ZnO}$ nanowires. We found that the lithium doping concentration is still lower than the native n-type donor background from oxygen vacancies, $\mathrm{Zn}$ interstitials, and possible hydrogen impurities. However, a clear dependency of n-type carrier concentration and lithium quantity is observed, which suggested lithium doping mechanism is a promising route towards p-doping in $\mathrm{ZnO}$ nanowires.

The Wu group; 1) Predicted that isoelectronic doping of conventional semiconductors can much enhance their thermoelectric thermopower; 2) Mapped and explored the phase diagram of a strongly correlated material $\left(\mathrm{VO}_{2}\right)$ over a phase space ten times broader than previously attained by taking advantages of the mechanical flexibility of nanowires. 3) Predicted that InGaN-based solar cell efficiency can be enhanced by grading the composition of active layers.

The Javey Group; Controlled assembly and/or growth of nanostructured III-V semiconductors on Si for subsequent doping studies and eventual device fabrication is rather channeling. This is in part due to the large lattice mismatch between Si and III-V semiconductors. Motivated by this challenge, we recently utilized an epitaxial transfer method for the integration of ultrathin layers of single-crystalline InAs on $\mathrm{Si} / \mathrm{SiO} 2$ substrates. As a parallel to silicon-on-insulator (SOI) technology, we use the abbreviation "XOI" to represent our compound semiconductor-oninsulator platform. Through experiments and simulation, the electrical properties of InAs XOI transistors are explored, elucidating the critical role of quantum confinement in the transport properties of ultrathin XOI layers. From TEM analysis, atomically abrupt interfaces between the ultrain thin single-crystalline InAs and amorphous $\mathrm{SiO} 2$ are observed. Importantly, a high quality InAs/dielectric interface is obtained by the use of a novel thermally grown interfacial InAsOx layer ( $\sim 1 \mathrm{~nm}$ thick). The fabricated FETs exhibit an impressive peak transconductance of $\sim 1.6$ $\mathrm{mS} / \mu \mathrm{m}$ at $\mathrm{VDS}=0.5 \mathrm{~V}$ with $\mathrm{ON} / \mathrm{OFF}$ current ratio of greater than 10,000 and a subthreshold swing of 107-150 mV/decade for a channel length of $\sim 0.5 \mu \mathrm{m}$. This presents a novel platform for reliability obtain nanostructured III-V layers for both doping and materials characterization studies. 
Surface-Selective Synthesis of Graphene Nanoribbons on Nanowire Templates

Principal Investigator(s): Yuegang Zhang

\section{Project Description}

Graphene has a great potential for future high-speed and low-power electronics due to its unique transport properties. Quantum confinement effect in a quasi-1D graph ene nanoribbon (GNR) could open a bandgap that is inversely proportional to the GNR width and strongly dependent on its edge atomic geometry (crystallographic orientation). The most straightforward method of e-beam lithography and plasma etching, however, has the difficulty to reach a width less than $10 \mathrm{~nm}$ and result in rough GNR edges that will reduce the carrier mobility and deteriorate the device performance significantly.

This project is to develop a new method to grow GNRs on a selected surface of a nanowire (NW) template. The surface-selectivity of GNR growth can be based on different lattice mismatch and epitaxial rates on different crystallographic planes of a single crystalline NW template. It can also be based on the cat alytic reactivity of a s pecific surface that promotes the graphene formation in a chemical vapor deposition process (CVD). By understanding the surface-selectivity and the nucleation/growth process of graphene synthesis on NW templates, we aim to achieve GNRs with well-defined width down to nanometer scale, controllable crystallographic orientation, and smooth edges. We will characterize the GNRs and verify the bandgap-GNR width relations predicted by various theoretical models.

Accomplishments

Direct deposition of graphene on various dielectric substrates is demonstrated using a single-step chemical vapor deposition process. Single-layer graphene is formed on thin copper films pre-deposited on dielectric substrates. The copper films de-wet and evaporate during or immediately after graphene growth, resulting in graphene deposition directly on the bare dielectric substrates.

We have grown GNRs utilizing the catalytic selectivity of the substrate material itself. By limiting the surface exposure of the catalytic reactive material, we can achieve selective catalytic GNR growth. Combined with controlled dewetting of metal catalyst during graphene growth, the graphene patterns can be directly synthesized on arbitrary substrates. We have demonstrated that submicron-scale graphene dots and ribbons can be grown using this method.

Other progresses include synthesis of Ni NWs using porous anodic alumina membrane as template. After CVD, Graphene nanoribbons were synthesized on the Ni NW t emplates as proved by Raman spectroscopy. We have also shown epitaxial gr owth of gra phene nanorribons on 1 ithographically defined $\mathrm{SiC}$ NWs. Further characterization and device fabrication is on-going. 
Bolometric Detectors for the Neutrino-less Double-Beta Decay Experiments

$$
\text { Stuart Freedman and Yury Kolomensky }
$$

\section{Project Description:}

The purpose of this LDRD project is aimed at developing advanced bolometric detectors for underground experiments searching for neutrino-less double beta decay and dark matter. The project plan includes constructing a cryogen-free He3He4 dilution refrigerator and associated hardware that can be used to operate bolometers at temperatures around $10-20 \mathrm{mK}$. The setup should be versatile enough to allow for the possibility of using other detection methods (scintillation detection, ionization detection, etc.) in coincidence with the phonon signal from the bolometer. The plan accommodates moving the compete system, including an associated facility for ultra-clean sample preparation, to an appropriate underground site. The system must be compact, self-contained, and portable.

\section{Accomplishments:}

In the first year of the LDRD we selected and purchased a dilution system, in complement with a refrigerator purchased elsewhere. Laboratory space in the basement of building 19 was prepared with the appropriate electrical and mechanical services required to operate the cryostat. The refrigerator has been delivered and is now being assembled in the laboratory space. We worked closely with the company engineers to make modifications to the standard cryostat support system that will enable the portability we require for our program. He3 gas is presently very difficult to obtain but the DOE nuclear physics office authorized us for $18 \mathrm{l}$ STP, which is adequate for the Triton 400 , and the gas has recently been delivered. The components ( $\mathrm{NaI}$ gamma ray detectors and single crystal cobalt sample) were obtained for a nuclear-orientation thermometer that is capable of precise temperature measurements below about $100 \mathrm{mK}$. The Cobalt crystal will be irradiated at a nuclear reactor to produce an implanted Co-60 source. A data acquisition system was constructed to allow us to make the required measurement of the temperature and serve as the host for the data from the bolometers and other detectors. 
Heavy Element Mass Analysis and Detector Capabilities

Principle Investigator(s): Kenneth E. Gregorich

\section{Project Description}

The purpose of this project is to do the initial design work and planning for a single-atom mass analyzer and detector station to be run in conjunction with the Berkeley Gas-filled Separator (BGS).

Since prediction in the 1960s of superheavy elements (SHE) stabilized by spherical nuclear shells, the production and study of SHE has been considered the Holy Grail of LowEnergy Nuclear Science. During the last 10 years, we have seen several claims of SHE production in Dubna, using ${ }^{48} \mathrm{Ca}$ projectiles with actinide targets. There have now been several independent confirmations of some of the Dubna SHE claims. However, the atomic number (Z) and mass number (A) assignments of all SHE isotopes are, to a large extent, based on comparison of measured and alpha-decay energies with those predicted using nuclear mass models. Before using the measured alpha-decay energies to refine the nuclear mass models, we must be certain that the $\mathrm{Z}$ and $\mathrm{A}$ assignments are correct (otherwise, refinements to the mass models will reinforce the incorrect $Z$ and $A$ assignments).

Technology being developed for the CARIBU project at ANL and the FRIB project at MSU will be used to build a single-ion mass analyzer, to be used with heavy and superheavy element isotopes after initial separation with the BGS. An RF gas-stopping cell will be installed at the BGS focal plane. Heavy element recoil ions will pass through a thin HAVAR window, and stop in high-purity He gas inside the RF gas-stopping cell. Because of the high ionization potential of the He, these heavy element ions will retain a 2+ charge state. Application of focusing $\mathrm{RF}+\mathrm{DC}$ fields will direct the heavy element ions toward an orifice. Once they near the orifice, they will be swept through with the gas flow. Subsequent gas skimming will result in a "beam" of 2+ ions which will be accumulated in a radio frequency quadrupole (RFQ) trap. Periodically, the ions accumulated in the RFQ trap will be sent through a mass analyzer to a lowbackground detection facility. Highly-efficient delivery of mass-separated heavy ions to a lowbackground detector facility on a 10-ms timescale will facilitate a broad, world-leading heavy element nuclear physics research program.

\section{Accomplishments}

Project scientist, Jacklyn Gates, has been spent a significant fraction of her time on this project. She has traveled to ANL to work with the CARIBU group, and become proficient in the use of the ion beam simulation software (SIMION). Using this software, design of the mass analyzer beamline has progressed. This design work has resulted in the Trochoidal Mass Analyzer concept. This is a modified Wien filter type mass analyzer system, that results in trochoidal ion trajectories in the filter, providing greatly increased dispersion and mass resolution.

Engineer, Tim Loew has been working on the detector facility design. At the end of the mass separator beamline, the ions will be stopped in a double-sided silicon strip detector (DSSSD) array. This DSSSD array will be surrounded with Ge clover gamma-ray detectors. Conceptual designs for positioning and holding the DSSSD and clover detectors are complete, and detailed design work has begun.

Work has progressed on a subcontractor order to have ANL build the RF gas-stopping cell and RFQ trap. Specifications for these items are being finalized. We expect to finalize the subcontractor order in early 2011. 


\section{A $100 \mathrm{~km}^{3}$ Neutrino Detector for Ultra High Energy Neutrinos Principal Investigator: Spencer Klein}

\section{Project Description}

The purpose of this project is to explore the feasibility of building a $100 \mathrm{~km}^{3}$ detector for ultra-high energy (above $10^{17} \mathrm{eV}$ ) astrophysical neutrinos on the Ross Ice Shelf in Antarctica. This detector would observe "GZK" neutrinos which are produced when cosmic-ray protons with energies above $4 * 10^{19} \mathrm{eV}$ interact with cosmic microwave background photons from the big bang. These neutrinos are the only way to probe the ultra-high energy universe at cosmic distances (more than 100 MPc from Earth), and thereby determine the source of ultra-high energy cosmic-rays. After examining several possible approaches, we chose to focus on radio-detection of neutrinos on the Ross Ice Shelf. There, a $570 \mathrm{~m}$ thick ice shelf sits atop the Ross Sea. The ice-water interface is a near-perfect reflector for radio waves. Radio waves from downward-going neutrinos will be reflected up to surface detectors. Moore's Bay, the chosen site is only $110 \mathrm{~km}$ from McMurdo, but protected from its radio emissions by a high bluff.

The ARIANNA detector will consist of a series of radio-detection stations which will observe coherent radio Cherenkov emission from the charged particles produced when these neutrinos interact in the ice. Frequencies from $100 \mathrm{MHz}$ to $1 \mathrm{GHz}$ are of the greatest interest. Signals may be observed with simple log-periodic dipole antennas (essentially TV antennas). Each station will consist of 8 buried antennas, arranged in an octagonal pattern, with a spacing of about 6 meters; from the relative signal timing and amplitude and radio spectral information, we can determine the neutrino energy.

\section{Accomplishments}

Our major accomplishment was to develop and deploy a prototype station at the proposed ARIANNA site. This prototype was based on a previous prototype, which was itself based on electronics for the ANITA balloon flight. However, we developed new preamplifiers, installed a new computer system, including a fair amount of new software, and upgraded many aspects of the system. This station meets most ARIANNA performance goals, although it is not suitable for mass production.

In December, 2009, Thorsten Stezelberger and Spencer Klein deployed this station at the proposed ARIANNA location. During a 9-day field season (during which we camped in tents), we deployed, calibrated and tested the station. We also bounced radio signals off the ice-water interface, to measure the ice thickness.

After we left, the station communicated via the internet (until the internet setup was removed, in late January, 2010), and then through an Iridium satellite modem. The power system worked well, with the solar panels providing adequate power until March, 2010, when the sun set. In addition to collecting data, the station took housekeeping records of the temperature and wind velocity. Although the station also had a wind generator, the wind at the site was inadequate to power the station.

The data quality appears to be very high. Except for some interference with the internet setup (which disappeared when it was removed), we appear to have reached the thermal noise limit, with no anthropogenic noise pickup.

In short, the radiodetection technique remains attractive, and the ARIANNA concept seems to work. 


\section{Project Report: Ultra-Sensitive Ge Detectors for Low-Background Physics Experiments Principal Investigator(s): Kai Vetter}

\section{Project Description}

The purpose of this project is to increase the sensitivity in the detection of rare physics processes such as dark matter interactions, neutrino-less double-beta decay, or coherent neutrino scattering. The detection of such processes requires ultra-low background and ultra-low electronics noise. The recent development in fabricating and operating low-capacitance p-type point contact (PPC) Ge detectors provides the potential to realize unprecedented noise levels in large, kg-size Ge detectors. This project aims at the evaluation of electronics readout schemes to realize ultra-low noise implemented with very low mass and low-radioactivity components required for the detection of rare processes. In addition, low-energy threshold and ultra-high resolution detectors of $1 \mathrm{~kg}$-scale can have a profound impact in atomic physics experiments as well as in nuclear forensics and safeguards applications.

\section{Accomplishments}

During the second year we were able to further evaluate technologies to improve the noise of PPC detectors conceptually, with our refined low mass front-end, and with a commercial system provided by our collaborators from Sandia National Laboratory (SNL). We were able to implement a new firmware for our digital data acquisition system that allows us to trigger at ultra-low noise while using optimal, i.e. long, shaping times which is difficult to achieve with conventional, analog systems. We are now able to operate the mini-PPC detector with a fully digital acquisition system and have achieved an energy resolution of about $110 \mathrm{eV}$ and a noise threshold of about $350 \mathrm{eV}$. The energy resolution is worse than originally measured which we believe is mainly due to the degradation of the detector itself over the last $>1$ years.

We have obtained a commercial Broad-Energy Ge (BEGe) detector from our SNL collaborators to study the limitations in its current implementation. We identified the source of the limited energy resolution of about $150 \mathrm{eV}$ to be potentially due to the $1 / \mathrm{f}$ noise of the detector, which is multiplied by the larger capacitance of the larger "point" contact being used by the commercial vendor. This represents an important result since several groups and scientific collaborations are planning on using this commercially available BEGe detector technology. It also confirms our approach of using a small (e.g. $\sim 1 \mathrm{~mm}$ ) diameter point contact with a very small capacitance JFET.

Based on the additional funding we received for this period we were able to acquire ultra-low background cryostats and larger volume high-purity Ge crystals necessary for a larger-scale demonstration and a potential deployment close to a power plant as originally planned.

We have developed concepts on how to reduce the noise of PPC detectors further. Operating the detector and readout at lower temperature, e.g. at $30 \mathrm{~K}$ instead of $\sim 80 \mathrm{~K}$ should not only reduce the thermal leakage current and improve the charge collection properties in the detector, it will allow us to use newly developed CMOS technologies with very low noise, potentially resulting in better than $40 \mathrm{eV}$ energy resolution.

We are just finalizing a manuscript on our findings for peer-reviewed publication. We are also planning to submit a proposal to DOE/NNSA to allow us to continue our work to ultimately deploy such a system close to a nuclear power plant and to observe the coherent neutrino scattering process. 
Theoretical Study of Nucleon Structure

Principal Investigators: Feng Yuan, Bowen Xiao

\section{Project Description}

The Understanding the structure of the Nucleon is a fundamental question in sub-atomic physics, and it has been under intensive investigation for the last several years. Modern research focuses in particular on the spin and the gluonic structure of the nucleon. They are key questions in current hadronic physics and also major driving forces for the RHIC and JLab programs. We propose an innovative research effort for theoretical study in this exciting area of strong interaction physics and focus on two topics: 1) the transverse spin physics and quark transversity distribution with the goal to extract the quark orbital angular momentum contribution to the proton spin; 2) the gluon structure at low Bjorken $\mathrm{x}$ and to study gluon saturation phenomena by carefully examining the gauge invariance and factorization properties in high energy processes.

\section{Accomplishments}

In order to understand the novel spin dependent observables in various high energy hadronic scattering processes, we calculated the transverse momentum dependent quark distributions in terms of the light-front wave function of nucleon. From these calculations, we demonstrated that the quark orbital angular momentum plays very important role to generate nonzero spin effects observed by the experiments. Further developments are needed to study the hadron tomography from these light-front wave functions. Studies along this line is under way.

Model independent analysis of the transverse momentum dependent quark distributions at large transverse momentum were performed based on perturbative Quantum Chromodynamics and the twist expansion of the quark-gluon correlation contributions to the relevant hadronic cross sections. We have demonstrated the consistency between the transverse momentum dependent factorization and collinear factorization approaches for all the leading power contributions to the Drell-Yan lepton pair angular distribution in hadronic reactions.

We systematically studied dijet production in various processes in the small-x limit and establish an effective kt-factorization for hard processes in a system with dilute probes scattering on a dense target. In the large-Nc limit, the unintegrated gluon distributions involved in different processes are shown to be related to two widely proposed ones: the Weizscker-Williams gluon distribution and the dipole gluon distribution. 


\title{
Physical Biosciences Division
}

\author{
Biological Methods for the Synthesis of Iron-Based Nanomaterials \\ Principal Investigator: Michelle Chang
}

\begin{abstract}
Project Description:
The purpose of this project is develop a deeper understanding of the chemistry that underlies the synthesis of iron-based nanocrystals by magnetotactic bacteria with the longterm goal of building biomimetic approaches to templating new spinel-type nanomaterials. Iron biomineralization is a complex yet insufficiently understood process that is key to understanding many important biological functions such as iron storage. Currently, there is very little molecular level information on biological magnetite synthesis. Furthermore, unlike more well-studied biomaterials based on calcium and silica, iron-based materials require redox chemistry during biogenesis. We thus seek to begin elucidating the mechanism of iron templating by magnetosome-associated proteins. We are still focused on studying the Mms family of proteins, thought to be directly involved with iron templating. These proteins contain two domains, consisting of an N-terminal silk-like motif thought to be involved in self-assembly of monomers as well as a C-terminal acidic domain that is proposed to bind and template metal ions. We will utilize a combination of biochemical and biophysical methods to study the mechanism of selfassembly and metal ion organization. In addition, we are also studying proteins involved in mineralization processes using in vitro biochemical studies.
\end{abstract}

Accomplishments:

We have developed a protocol for the preparation of purification of several of the Mms family proteins and now have quantities of Mms6, Cys-Mms6, Mms6-C term, Mms6-N-term, Mms7, Mms7-C term. These different constructs will allow us to carry out biophysical studies on the full-length protein compared to the N-terminus (involved in self-assembly) and the Cterminus (involved in mineral binding). We have been able to monitor concentration-dependent changes in protein structure and monomer assembly by both circular dichroism spectroscopy and dynamic light scattering. Using surface plasmon resonance measurements, we are also able to visualize the aggregation of Cys-Mms6. The salt dependence indicates that Mms6 interacts with itself via hydrophobic interactions. Binding studies with monomeric iron indicated that these proteins had little affinity for ferric or ferrous ions. We are now pursuing calorimetry measurements with these proteins using magnetite nanoparticles. These nanoparticles have been prepared and characterized by TEM. In addition, we are developing antibodies to utilize for coimmunoprecipitation studies to monitor protein-protein interactions involved in magnetosome biogenesis. We have also developed heterologous expression platforms for production of magnetosome-associated proteins in E. coli and are currently assaying their biochemical function in vitro. 
Engineering environmental sensitivity in an artificial cell Principle Investigator: Daniel A Fletcher

\section{Project Description}

This project develops a system for studying in vitro expression of sensory proteins using a giant unilamellar vesicle platform. Biological systems are exquisitely sensitive to their local environments, using information such as osmolarity, light intensity, and $\mathrm{pH}$ to dynamically alter their behavior. In contrast, non-biological systems can have unique chemical, electrical, and physical properties, such as those found in nanostructured materials, but they are notoriously unresponsive in organization and behavior to their local environments. For applications ranging from development of adaptive materials to directed self-assembly of nanoparticles, a method for harnessing the environmental sensitivity of biological systems in a platform that is compatible with non-biological materials is needed. In this project, we propose to develop such a platform by reconstituting an environmentally sensitive two-component signaling system in synthetic vesicles using a newly developed encapsulation technology. Working with the E. coli osmolarity sensor EnvZ-OmpR as our test case, we will demonstrate the ability of a synthetic assembly to sense and respond to external conditions, setting the stage for the creation of responsive hybrid materials based on engineered gene expression.

\section{Accomplishments}

In the second year of this project, we accomplished three major goals that are required to increase the functionality and complexity of vesicles formed with the encapsulation technology: (i) incorporation of oil-insoluble lipids into the vesicle membrane, (ii) incorporation of transmembrane proteins into the vesicle membrane, and (iii) control of lipid and protein asymmetry in the vesicle membrane. Cell membranes have each of these three properties, but no reconstitution technique to date has been able to achieve all three at the same time as controlling vesicle contents. Using the microfluidic encapsulation technology developed as part of this project, we have demonstrated that such control is possible.

Microfluidic encapsulation is based on deforming a lipid bilayer formed between two aqueous droplets into a vesicle with a liquid jet. In the previous year, we demonstrated that protein expression can be accomplished inside of the vesicle, yet we have relatively little control over membrane properties. To address this limitation, oil insoluble lipids such as the charged signaling lipid $\mathrm{PIP}_{2}$ and PEGylated lipids were incorporated into membranes using an SUV fusion approach. In this approach, SUVs fuse with the oil-water interface and form lipid monolayers around aqueous droplets, which can be brought together to form lipid bilayers. In the absence of oil dissolved in the lipid, the membrane formed will depend on the lipids in the SUVs, which can be oil-insoluble. A similar strategy was used to incorporate SNARE proteins into lipid monolayers and then bilayers. These SNARE proteins contain single helicies that anchor the protein into the bilayer and can be transferred to an oil-water interface from SUVs. We demonstrated the functionality of the SNARES by reconstituting exocytosis of SUVs. If different SUVs containing different lipids or membrane proteins are used in the two aqueous droplets, membrane asymmetry can be accomplished. This ability to control the molecular composition of the membrane demonstrates that vesicles can be used as platforms for not only protein expression but also insertion of functional membrane proteins. 


\title{
Metafluxomics of a phosphorus removing microbial community
}

\author{
Principal Investigators: Héctor García Martín and Aindrila Mukhopadhyay
}

\section{Project description}

We propose to develop a method to measure intracellular metabolic fluxes for a complete microbial community. Whereas measurement of intracellular fluxes (i.e. the rate at which molecules proceed through a reaction per unit of time) for all metabolic reactions in an organism has been so far limited to pure cultures (fluxomics), measurement of metabolic fluxes for a full microbial community (metafluxomics) would be a first in scientific literature and a logical progression in the advent of metagenomics, metatranscriptomics and metaproteomics technologies.

We will develop proteomic methods to measure and assign intracellular metabolic fluxes to each of the species in a microbial community. We will use this information as a stepping stone to produce quantitative predictive models of microbial community metabolism and its impact on its environment. The study of internal metabolic fluxes is the optimal basis for community modeling since they include knowledge of growth rates (which are just fluxes to biomass) and the biochemical impact on the environment (which is just the incoming and outgoing metabolite fluxes into/to the environment).

The project will unfold in four different phases of ascending complexity: the first step will involve computationally testing that the same results are obtained with the proteomic method and the standard amino-acid based method. The second one will involve the experimental check that, for E. coli, the same results are obtained using both methods. The third phase involves checking that from the combined lysate of $S$. cerevisiae and E. coli grown separately, it is possible to obtain flux distribution and assign it to each of the species. The final phase involves using the method with a full microbial community, in this case Enhanced Biological Phosphorus Removal (EBPR) sludge, a microbial community of industrial significance used to remove phosphorus from wastewater.

\section{Accomplishments}

Our most significant accomplishment so far has been to computationally compare the new proteomic-based method with the traditional amino acid-based approach and show that similar flux profiles are obtained (phase 1). We are now working in thoroughly understanding what kind of information loss is incurred by using this method in comparison with the standard approach and if it can be ameliorated by properly tuning the experimental parameters. In order to do this we have developed an information content measure and we are using it to explore the parameter space.

Furthermore, we are now analyzing the experimental data for a pure culture of $E$. coli (phase 2) and have also developed a sampling scheme for the EBPR system which will provide a complete data set for flux determination for this microbial community. 


\section{Applications of Hybrid Live Cell - Synthetic Devices for Cancer Research Principal Investigator(s): Jay T. Groves}

\section{Project Description}

The purpose of this project is to utilize the hybrid live cell - supported membrane platform to reconstitute numerous functional aspects of cancer. In particular, the EphA2 receptor tyrosine kinase is overexpressed and functionally altered in $40 \%$ of human breast cancers, and recent evidence indicates that treatment with the ephrin-A1 ligand can reverse tumorigenesis. Since both Eph receptors and their ephrin ligands are natively membrane-bound, this signaling system is ideally suited to reconstitution into the hybrid live cell-supported membrane configuration.

We will first develop a display strategy to present laterally mobile ephrin-A1 on the surface of a supported membrane to EphA2-expressing breast cancer cells. Then we can study how the lateral organization, cluster size and mobility of ephrin-A1 ligands affect EphA2 signaling. These are all attributes of ligand presentation that exist in vivo, and the supported membrane platform allows us to access them in vitro. We can then examine correlations in EphA2 signaling across a panel of human breast cancer cell lines and relate changes in the molecular mechanisms of signaling to disease characteristics across the entire cell line library. In this way, controlled in vitro experiments on live cultured cells can be related to disease pathology in humans.

\section{Accomplishments}

The core preliminary observation that large-scale spatial reorganization of the EphA2 receptor occurs concomitantly with ephrin-A1 ligand triggering has been confirmed. More importantly, we have confirmed that mechanical restriction of EphA2 transport, using nanofabricated barriers to transport on the supported membrane surface, materially alters signaling outcome, in terms of metalloprotease recruitment and cytoskeleton morphology.

To probe the generality of this spatial reorganization phenotype, we measured EphA2 transport across a library of mammary epithelial cell lines. We found that there was a strong correlation between EphA2 translocation and the ability of a cell to invade foreign tissue. A system-wide correlation of EphA2 transport scores with all available protein and gene expression data across this library revealed several genes and proteins that are associated with this phenotype which could serve as therapeutic targets for receptor reorganization.

We have already obtained some follow-on funding to extend limited portions of this work, including probe of specific mechanical aspects of signaling in cancer, from the National Cancer Institute and the Department of Defense Breast Cancer Research.

We successfully completed goals of the 2010 LDRD and have published these findings in 'Restriction of Receptor Movement Alters Cellular Response: Physical Force Sensing by EphA2', Khalid Salaita, Pradeep M. Nair, Rebecca S. Petit, Richard M. Neve, Debopriya Das, Joe W. Gray, and Jay T. Groves Science 12 March 2010 327: 1380-1385. Another publication detailing the methods used is in press at Nature Protocols. We have also secured competitive extramural funding to continue this project from the DOD Idea Award. 


\section{Advances in Standardized, Scar-less, Sequence-Independent Cloning Methods Principal Investigator(s): Nathan J. Hillson}

\section{Project Description}

A diverse array of biological endeavors, spanning from the production of clean renewable sources of biofuels and chemicals, to environmental stewardship, to the development of cancer therapies, share in common labor-intensive molecular biology procedures. There is significant promise that standardization, coordination, and consolidation of multiple independent cloning projects could be achieved though the use of automated design and with methodological advances. Such advances would be driven by software improvements as well as by redesigning protocols with robotics and automation integrated into process workflows.

This project will accelerate progress towards these advances in standardized, scar-less, sequence-independent cloning methods. A graphical user interface will be developed that will facilitate and standardize DNA assembly design for molecular and synthetic biologists. The resulting experimental designs will be aggregated into 96-well plates, enabling a single researcher (utilizing multi-channel pipettes and/or liquid-handling robotics) to perform the synchronized cloning tasks of several people. As part of the workflow optimization process, cost/benefit analyses will be investigated. Following preliminary screening, DNA constructs will be submitted for sequencing and automated analysis; successful clones will be delivered to endusers.

\section{Accomplishments}

A pre-requisite to optimizing our automated DNA assembly process workflow is the identification and evaluation of methodological alternatives for throughput-limiting or costintensive operations. DNA purification via gel electrophoresis is a labor-intensive step in our current workflow. As an alternative, we have developed conditions for isolating desired PCR products from primer-dimer contaminants with a $\mathrm{PEG} / \mathrm{NaCl}$ precipitation step, which can be automated in 96-well format utilizing liquid-handling robotics and a centrifuge.

We have refined our process workflow while simultaneously positively contributing to the construction of DNA libraries that are valuable to researchers as they pursue renewable sources of biofuels and chemicals. We have constructed a combinatorial library of fluorescent proteins that report the formation of engineered micro-compartments that will eventually be utilized to bolster biofuel productivity. We have completed designs for two (and are in the process of completing designs for two additional) combinatorial DNA libraries, and have begun constructing the components required to assemble the first of these. These libraries will be constructed and used to inform our process workflow over the course of the next year.

Improvements have been made to the graphical user interface software that aids the design of the DNA to be assembled. A significant investment has been made in refactoring the software code base, which will facilitate its further development and enhance its maintainability. New features added to the software include providing access to design specification rules, a proper replicate part information sharing model, and improved formatting of the resulting design output files. A web server has been dedicated to the developed software tools, which are available (http://j5.jbei.org) at no cost to academic, non-profit and government institutions. 


\section{Light-boosted Fermentation in the Yeast Saccharomyces cerevisiae \\ Principal Investigator: Jan Liphardt}

\section{Project Description}

Our goal is to discover the fundamental biophysical principles governing energy fluxes in living cells. For example, we wish to understand how energy generated in one organelle in the form of a transmembrane potential can be optimally delivered to energy consuming reactions in the cytoplasm. We have built a light harvesting $S$. cerevisiae yeast, which allows us to optically deliver controlled amounts of energy to single cells. Our research goal is to perfect this unique bioenergetics research platform, and to determine the effect of illumination on a variety of cellular fluxes such as glycolysis. These studies will reveal the energetic balance of anaerobic growth and the degree to which certain cellular processes are energy limited, rather than carbon limited. Moreover, light harvesting yeast may have commercial significance, since its ability to convert input carbon to a variety of fuels or fuel precursors may be increased compared to natural systems.

\section{Accomplishments}

We have created various strains of the $S$. cerevisiae yeast expressing the light powered proton pump PR. Illumination of certain PR+ yeast strains alters their metabolism, as judged by carbon utilization, $\mathrm{CO}_{2}$ output, and EtOH production. The research supported by this LDRD yielded several discoveries:

(1) Some forms of PR have the ability to reconfigure the cell's internal membranes, so that they fold back on themselves and create membrane stacks reminiscent of light collecting structures in plants and in the photoreceptors in our eyes. This may be the first example of the synthetic design of cellular ultrastructure to boost desired metabolic processes.

(2) We also discovered that we can create a second "shape" mutant, which we call "bundles of grapes." We suspect, but have not yet proven, that by adjusting the dimerization affinity, we can generate membrane stacks or "bundles of grapes."

(3) Although we are able to modify cell architecture, we were not able to do so in a precisely controlled manner.

The work performed in this LDRD has exposed synthetic biology's lack of fundamental understanding of (1) organelle architecture and (2) protein targeting to organelles. We conclude that one key barrier to success and further refinement of engineered light-collecting organisms is the lack of understanding of heterologous protein targeting to specific organelles. 


\title{
Engineering of Drought and Heat Tolerance in Bioenergy Crops
}

\author{
Principal Investigator(s): Henrik Vibe Scheller
}

\section{Project Description}

The aim of the project is to engineer stress tolerant plants that accumulate the thermo and osmoprotectant mannosylglycerate in response to abiotic stress. Bioenergy crops growing on marginal lands will often be exposed to such stress factors and will be particularly important targets for engineering of these traits. Mannosylglycerate may protect plants against several different kinds of stress, and in addition to drought and heat stress, salt stress and cold stress will be investigated in the most promising plant lines.

Plants (arabidopsis, Brachypodium and rice) will be transformed with constructs encoding mannosylglycerate synthase from the lycophyte Selaginella moellendorfii under control of constitutive and stress-induced promoters. Constructs using the bifunctional mannosyl-phospho-glycerate / mannosyl-phospho-glycerate phosphatase from Dehalococcoides ethenogenes will also be tested as an alternative route of synthesizing mannosylglycerate. The accumulation of mannosylglycerate and other metabolites during and after stress will be analyzed and related to stress tolerance of the obtained plants.

Accomplishments

The gene encoding mannosylglycerate synthase gene from S. moellendorfii was codon optimized for efficient expression in plants. The synthetic gene was engineered into vectors for transformation of different plants with and without tags. Arabidopsis was transformed with constructs driven by the constitutive cauliflower mosaic virus $35 \mathrm{~S}$ promoter or a heat-shock inducible promoter from Arabidopsis. The $35 \mathrm{~S}$ driven constructs were also used in tobacco transient transformation. Rice and Brachypodium were transformed with constructs driven by ubiquitin promoter. Transgenic Arabidopsis and rice plants were generated and expression of the gene confirmed. Plants expressing an HA-tagged version of the enzyme were confirmed by immunoblotting to express the protein.

Metabolites were extracted from plants and analyzed by LC-MS. Mannosylglycerate was detectable in all of the transgenic plants. None of the plants showed any developmental or morphological phenotype. The content of mannosylglycerate varied between different plant lines but in general the levels were relatively low. Preliminary stress experiments did not show a strong protective effect in the transgenic plants. However, detailed stress experiments will be conducted when plants with higher levels of accumulation have been identified or developed.

We are currently investigating the levels of key metabolites in the mannosylglycerate biosynthesis pathway to identify the limiting factors for the accumulation of the compound and develop strategies for further enhancing the production.

The bifunctional mannosyl-phospho-glycerate / mannosyl-phospho-glycerate phosphatase gene has been codon optimized for plants and synthesized. Arabidopsis plants transformed with the bifunctional gene construct have been generated but not yet analyzed. 


\section{The Control of Intraflagellar Transport in Chlamydomonas Cells \\ Principal Investigator(s): Ahmet Yildiz}

\section{Project Description}

The purpose of this project is to observe how microtubule motors interact with each other and how cells control the activity of these motors to coordinate bidirectional transport. We use Chlamydomonas intraflagellar transport (IFT) as a model system and apply single molecule imaging techniques to address these questions. These studies have enabled us to dissect how many motors carry a single cargo in IFT and whether opposite polarity motor proteins, kinesin and dynein, coordinate with each other or compete in a tug-of-war manner during the transport. The data will further enable to solve the mechanism by which cargoes travel long distances, reverse their direction, and the exchange of motors at the turnaround zones. Using genetically modified strains and superresolution imaging in live cells, we aim to discover the enzymes that play a role in regulation of motor protein activity. These assays will present new insight on molecular mechanism of intracellular cargo transport in general and provide fresh insight on several types of human diseases.

Accomplishments:

We have examined the motion of individual IFT cargos. We observed that IFT particles move anterogradely and retrogradely. We then examined the movement of kinesin motors. Surprisingly, we only observed kinesin motors to move towards the tip. Backward motion of these motors towards the base was not observed. The results can be interpreted as kinesin motors are used only for once in IFT and exocytosed or degraded at the tip region. Alternate possibility is that it is the KAP subunit not the kinesin heavy chain released from the cargo at the tip. To clarify this issue, we will label the kinesin heavy chain and repeat the tracking measurements. We will also collaborate with George Witman Lab (UMass) to tag IFT dynein with CFP and track kinesin and dynein motion along the flagella simultaneously to further test the model.

By using multicolor tracking microscopy, we have shown that flagellar membrane glycoprotein 1 (FMG1) is transported by IFT machinery forward and backward along the length of flagella. The observation provided us novel tools to track the motion of IFT particles at high resolution. We have observed that IFT particles move by taking $8 \mathrm{~nm}$ steps towards both ends of the flagella, in agreement with in vitro measurements of kinesin and dynein step sizes. Movement of the beads was unidirectional over large distances. These results indicate that IFT differs significantly from other bidirectional transport systems. The motors responsible of IFT can be switched on and off quickly at the turnaround zones so that they do not compete against each other to determine the direction of cargo transport.

To quantify how many motors carry a single cargo, we have studied IFT movement under force. We have observed that the number of moving beads depends heavily on the amount of antibody on the bead surface. Stall force measurements have shown that cargo movement seizes at 20-30 pN opposing forces. The data indicates that on average 4-5 kinesin and dynein motor proteins carry a single cargo (motor forces are additive at low motor numbers).

We also discovered a surprising relationship between IFT and cell gliding motility along solid surfaces. Chlamydomonas cells can glide linearly by orientating their flagella at $180^{\circ}$, but the mechanism of force generation remained unresolved. Earlier studies have shown that mutations in either IFT motors or FMG1 stop gliding. We have tracked individual IFT particles under conditions that allow cell-gliding motility. We observed that retrograde IFT particles transiently got stuck to the glass surface and the cell starts gliding towards the opposite direction of the IFT particle at the same time. In the majority of our data, more than one retrograde IFT are involved at the same time to produce higher force for gliding. 
The development of reusable software modules for the analyses of small angle scattering data

Peter Zwart, Alexander Hexemer, Paul Adams

\section{Project Description}

The purpose of this project is to develop reusable software modules for the analysis of small angle scattering data (SAXS). SAXS is an import biophysical technique allowing one to probe structure and dymanics of macromolecules in near native environments. The project is focused on implementing know algorithms as well as developing new computational methods for the analyses of such data.

\section{Accomplishments}

Over the course of the project we have accomplished the following major milestones. First of all, we have developed structure refinement techniques allowing one to determine structural changes incurred on the protein in solution as compared to their crystal structure. This information is highly essential for the interpretation of drug-induced conformational changes and can be a potential tool in drug discovery efforts. The second major accomplishment are improvements in shape recovery procedures. Shape recovery algorithms allow one to determine a low-resolution molecular shape of proteins in solution from SAXS data only and provide clues on the biological function. Existing shape recovery algorithms are computational intensive procedures that can take hours to days to fully converge. We have developed a fast method for shape recovery that produces approximate shapes within minutes.

\section{KHIRIX DHIKDVEHQDMDCECOIRUMUQJ प\$ IQXP EHURICIIHHQWDEVIWYHEHQ MUDJ IRXUSLRFHGXUMDOGSLRYGHGXYHXOHOEDFN}




\section{LB08048}

\section{Physics Division}

Development of Multi-Modular Assemblies with Reduced Material and Services for Tracking at Future Colliders

Principal Investigator(s): M. Gilchriese and C. Haber

\section{Project Description}

Future silicon tracking detectors, such as for a Super Large Hadron Collider (SLHC), require the development of multi-modular mechanical support structures with integrated cooling and electronics on an unprecedented scale. The purpose of this project is to study a concept which can meet the requirements of such tracking systems. The concept is based upon thin, low mass, stiff composite laminated structures which embed both high performance cooling and electrical distribution of high bandwidth signals and efficient power distribution methods.

The project consists of two principal aspects. One of these is the design of integrated mechanical/cooling structures that could be applicable to all elements and regions of a future silicon tracking system for the SLHC. These require both design studies (analytical calculations and finite-element calculations) and the fabrication of a number of prototypes to explore and test the design concepts. The second aspect is to assemble and test the electrical performance of prototype integrated structures using silicon strip modules.

\section{Accomplishments}

The conceptual design for integrated mechanical/cooling/electrical structures for future pixel detectors was advanced significantly. Prototypes were constructed and tested to verify reliability after extensive thermal cycling (to less than $-35 \mathrm{C}^{\circ}$ ), after irradiation (up to $1 \mathrm{GRad}$ for critical components) and after combined irradiation and thermal cycling. Full-scale mechanical/cooling prototypes were fabricated and tested (thermally) to demonstrate the feasibility of integrating electrical distribution within the mechanical structure. Basic properties of the critical component of the structure (recently developed low-density carbon foam) were measured. These properties were used in finite element analysis of the prototypes to predict thermal and mechanical performance. Good agreement was achieved between measurements and predictions. The overall conclusion is a validation of the proposed design concept for integrated mechanical/cooling/electrical structures for future large scale pixel detectors.

In the prior periods we reported on the fabrication and test of a multi-modular electrical stave structures. Test and further fabrications have continued over the last year as well. We continued to study and demonstrate a large scale application of serial powering, with good electrical performance. In order to embed the multi-module system in a practical configuration various aspects of data transmission, interface, and multiplexing must be demonstrated. In collaboration with SLAC and UK colleagues an interface chip was developed. We developed a test system and packaging arrangements to demonstrate the performance of this device and tested and distributed large numbers. Tests of these chips exposed a number of technical issues, which were studied and addressed through circuit modifications. We also complete the design and fabrication of an interface circuit board to connect to the external DAQ, control, and powering.

Multi-drop data transmission was demonstrated on bus cables as well now utilizing the aforementioned interface chips. We wrote additional firmware and software to operate this system, and tested a new series of boards as well.

As a result of this LDRD supported activity, both the mechanical/thermal and electrical aspects of integrated multi-modular structures have been put on a firm technical foundation. 
Study of History of Global Surface Temperatures

(Berkeley Earth Surface Temperature)

Principal Investigator(s): Richard A. Muller

\section{Project Description}

The most important indicator of global warming, by far, is the land and sea surface temperature record. This has been criticized in several ways, including the choice of stations and the methods for correcting systematic errors. The Berkeley Earth Surface Temperature study sets out to do a new analysis of the surface temperature record in a rigorous manner that addresses this criticism. We are using over 39,000 unique stations, which is more than five times the 7,280 stations found in the Global Historical Climatology Network Monthly data set (GHCN-M) that has served as the focus of many climate studies.

Our aim is to resolve current criticism of the former temperature analyses, and to prepare an open record that will allow rapid response to further criticism or suggestions. Our results will include not only our best estimate for the global temperature change, but estimates of the uncertainties in the record.

Accomplishments

We have reached the first major milestone of the Berkeley Earth Surface Temperature project. We have already collected all of the data and merged it into a complete data set of approximately 1.6 billion records. We are currently in the process of developing a system that will update our database regularly as new data becomes available. We are also working on making the data easily accessibly to other scientists and the public - and we hope to have that finished by the end of the year.

In the meantime, our core team has already begun the analysis. To begin, we are working with a $2 \%$ sample, which will help us to refine and de-bug our analysis techniques. We hope to have some initial findings on the full data set by the end of 2010 - which will give us an interesting peek at whether we find more, less, or the same amount of global warming as previous studies.

Finally, we have also been working hard to inform the key climate scientists, organization heads, bloggers, the media, and other influencers in the global warming debate regarding our work. The vast majority of people we have spoken to have been very supportive, and by extending the engagement of these "stakeholders" we hope to ensure that we are judged by the quality of our methodology, not simply the headline results that we find. We believe that this will be a critical element of ensuring that our work has maximum benefit and influence. 


\section{Theoretical Studies of Dark Matter Beyond the Standard Model} Principal Investigator: Yasonori Nomura

\section{Project Description}

There is incontrovertible evidence for an unknown form of "dark matter" comprising roughly $25 \%$ of the universe, while no possible dark matter candidate can be found within the Standard Model of elementary particle physics. On the other hand there is a tantalizing clue that weakly interacting particles with masses around the scale of electroweak symmetry breaking (i.e., WIMPS), have just the right ratio of mass to interaction strength required to populate the universe at precisely the density required for dark matter. Dark matter may then be tightly linked to the puzzles about the origin of mass (AKA electroweak symmetry breaking) that are not satisfactorily addressed in the Standard Model, and that will soon be addressed at the LHC.

\section{Accomplishments}

Following my previous work, I have constructed a model of dark matter which naturally explains astronomical anomalies that have been observed in cosmic ray experiments (PAMELA, FERMI, H.E.S.S.) [1]. This model naturally arises from theory of supersymmetry breaking at low energies, which has virtues of avoiding the notorious supersymmetric flavor problem as well as the gravitino problem. Dark matter is a composite particle arising in the sector breaking supersymmetry dynamically, and cosmic ray signals arise by slow decays of dark matter, which is caused by the mechanism analogous to proton decay in unified theories. The model leads to a number of interesting signals at collider experiments, including the LHC.

I have also developed theories in which WIMP dark matter arises as a result of environmental selection in the multiverse [2]. It is known that small cosmological constant (dark energy) can be explained by environmental selection. The existence of dark matter may also be explained similarly. This opens up new possibilities for dark matter---weak scale supersymmetry is broken at high energies, leaving the wino or the Higgsino-bino at low energies. The scenario leads to interesting signals at both direct and indirect dark matter detection, as well as at colliders, and may be tested in next generation experiments.

I continue to develop theories of dark matter which are related with ideas in particle physics, e.g. supersymmetry, extra dimensions, and the multiverse. I have recently introduced a new framework for breaking supersymmetry in the setup motivated by the string landscape. In this framework, supersymmetry is broken multiple times in mutually separated sectors, which leads to novel light fermions called "Goldstini" [3]. We found that these Goldstini affect cosmology in various ways. In particular, we noticed that the Goldstini can be dark matter of the universe [4]. This scenario can be realized without conflicting with the standard cosmology, including big-bang nucleosynthesis and baryogenesis. We presented a scenario in which bigbang nucleosynthesis is made compatible with thermal leptogenesis---arguably the simplest mechanism for baryogenesis. This scenario was impossible in the minimal supersymmetric theories, but here made possible due to the existence of the Goldstini. 


\section{R\&D for Fast, Low-Noise CCD Readout and Single Photon Detection Capability}

Natalie Roe, Physics Division

Peter Denes and Steve Holland, Engineering Division

Project Description: We proposed an R\&D project aimed at designing advanced CCDs based on the current state-of-the art fully depleted p-channel CCD that was developed at the LBNL Microsystems Laboratory (MSL). The FY10 LDRD funding proposal goal was the fabrication and test of a lot of wafers with a new layout that includes twelve new CCD designs that were developed under the previous year's LDRD proposal. The new CCD designs include devices capable of single-photon detection, faster readout, and better noise performance and drive capability. Also, exploration of advanced wafer stepper photolithography techniques was investigated. Applications of these technologies include the next generation of ground- and space-based astronomical surveys to study dark energy, soft x-ray detection at light sources including the ALS, LCLS and other photon sources, and night vision and radiological surveillance for homeland security.

Accomplishments: Twenty wafers have been fabricated through DALSA Semiconductor Inc. using a new layout for a six-inch wafer that includes a total of 28 CCDs. Some devices are controls based on previous designs and others are duplicates. The wafer layout includes the following new device designs:

1) Two-stage output amplifier for fast readout and higher drive capability

2) Small (1 um) contacts to channel stops to improve the understanding of channel stop effects and explore advanced photolithography capabilities at DALSA Semiconductor

3) Buried contact amplifiers to reduce capacitance and improve read noise

4) Same as 3) but with smaller buried contacts

5) Floating gate amplifiers to enable non-destructive charge sensing

6) Segmented large format CCD optimized for a next generation dark energy survey

7) Same as 5) but with variation in the readout architecture

8) Charge multiplying CCD for single photon detection

9) Same as 8) but with new architecture for multiplication cell

10) Fast CCD in large format with multiple readouts and frame-store capability for x-ray detection in light source applications

11) Same as 10) but in smaller format, no frame-store

12) Same as 11 but with a hole in the middle to provide for beam passage

Due to the many different designs, several requiring customized process flows, the lot took longer than usual to complete fabrication at DALSA. The first wafers were received early in September, 2010 and device testing was initiated immediately. Designs 6, 8, 9, 10 and 11 have been packaged and tested. Preliminary results are very encouraging. The large format CCD optimized for next generation dark energy surveys is working well and precision testing is in progress by our collaborators at Fermi National Accelerator Lab. The fast CCDs designed for soft x-ray detection at light sources are also functioning as expected, and prototype instruments incorporating these new devices are being assembled at LBNL using ARRA funding. The charge multiplying CCDs did not perform as expected and the cause is under investigation. Measurements indicate that an error in fabrication occurred at DALSA, and they have agreed to replace the affected wafers. 


\author{
Decoding Dark Energy with Weak Gravitational Lensing \\ Principal Investigators: George Smoot, Alexie Leauthaud
}

Project Description

Understanding the nature of dark matter and dark energy represents two outstanding challenges for the new century. Whereas compelling evidence suggests that dark matter is semi-relativistic and collisionless, thus guiding our quest for the appropriate particle, the general properties of dark energy remain far more elusive. We propose a comprehensive study of weak lensing as one of the most powerful means to study dark energy. We will carry out this study in two steps: first by analyzing the ability of weak lensing to directly detect massive clusters and second, by comparing these results to various other weak lensing statistics that are also sensitive to the dark energy equation of state.

Accomplishments

Firstly, in 2010 we have published a study of the weak lensing signal around groups and clusters in the COSMOS field. Groups have been stacked according to their X-ray Luminosity $\left(L_{X}\right)$ which allows for a better understanding of the relationship between the hot intra-cluster gas and dark matter halos. This study is an important step forward towards better measurements of the abundance of galaxy clusters as a function of halo mass. These results have been published in the Astrophysical Journal (Leauthaud et al. 2010).

Secondly, in 2009-2010, we have constructed a novel method by which to correct imaging data for the undesirable effects of Charge Transfer Efficiency which can affect the measurement of galaxy shapes. This is an important step forward towards designing future space-based weak lensing mission (Rhodes et al. 2010, Massey et al. 2010).

Thirdly, in collaboration with UCB graduate student Matt George, we have been investigating the effects of mis-centering on the COSMOS group weak lensing signal. We have found that mis-centering effects can be clearly detected through weak lensing and we can use this effect as a tool to improve the determination of the centers of groups and clusters. We are currently working on a series of papers that will describe these results (George et al. in prep).

Finally, we have put substantial efforts into extending the COSMOS results to larger statistical samples. In 2010, we wrote a successful SDSS-III ancillary proposal to target an ensemble of 4000 groups and clusters for spectroscopy in the so called "Stripe 82" equatorial region (P.I Leauthaud). In 2010, we also successfully obtained CFHT weak lensing data over the same field $\left(170\right.$ degrees $\left.^{2}\right)$ to perform weak lensing measurements. This new weak lensing data is currently being reduced by graduate student Matt George and other collaborators. In September 2010, we have also submitted a proposal to obtain IR data over the same field to improve photometric redshift estimates. The combination of these new data sets obtained in 2010 will enable us to extend our work in COSMOS by studying a larger sample of groups and clusters of galaxies with weak gravitational lensing. 


\section{LB08046}

Calibrating Baryon Oscillations for Future Experiments PI(s): Nikhil Padmanabhan, Martin White, David Schlegel

Project Description: The baryon oscillation method is now an essential element of all programs to measure the expansion rate of the Universe and constrain the properties of "dark energy". The ongoing BOSS survey will constrain the expansion at $1 \%$ at intermediate redshifts, the next generation of surveys will constrain the expansion to sub-percent at multiple redshifts and the planned WFIRST satellite would achieve $\sim 0.1 \%$ constraints. This project provided essential the theoretical support for these experiments, by quantifying possible systematic errors and developing methods for optimally extracting the baryon oscillation signal from these data sets. We described the effects of the (a) nonlinear clustering of the dark matter, (b) halos and galaxies, and (c) redshift space distortions on the acoustic feature using large numbers of N-body simulations of varying resolution. Combining these simulations with perturbative techniques, we developed an analytical framework that captures the effects at the fidelity required by the next generation of surveys.

Accomplishments: We made significant progress on understanding how the nonlinear clustering and halo bias affect the baryon acoustic feature. We ran $\sim 1,000 \mathrm{Gpc}^{3}$ of Nbody simulations (the next generation of experiments will survey $\sim 10 \mathrm{Gpc}^{3}$ of the Universe), across very different cosmologies and for a variety of simulation parameters. We demonstrated that our current simulation techniques are accurate enough to correctly model the baryon oscillations and used these simulations to demonstrate that the effect of nonlinear clustering on the baryon oscillations is accurately described by second-order (1-loop) perturbation theory for both biased and unbiased tracers. This allowed us to construct a analytic model for baryon oscillations in the nonlinear regime that is accurate to the $\sim 0.1 \%$ level necessary for future surveys. We additionally quantified the requirements of "reconstruction" methods (designed to undo some of the effects of nonlinear clustering) on the survey parameters, which has important implications when optimizing the design for the next generation of surveys. Preliminary investigations of acoustic oscillations in the intergalactic medium were reported, a potentially game changing technique. In addition, we have improved our analytic understanding of how these methods work and what their regimes of validity are. These efforts have suggested improvements that can be made to such methods, an area for future research (with NASA funding now in hand). They have also allowed us to focus our simulation resources on the most challenging areas while using the analytic methods where they are applicable. This makes us more efficient and has had spin-offs beyond just this project, since simulations are now integral to most dark energy experiments/methods. 


\section{Cross-Divisional}

\section{Integrated Tools in Multiscale Imaging}

PI: Manfred Auer (Life Sciences Division) co-PI: Jan Liphardt (Physical Biosciences Division)

Phil Hugenholtz (Joint Genome Institute),Terumi Kohwi-Shigematsu (Life Sciences Division) Project description.

The overall goal of this project is to invent and apply a new generation of imaging technologies to biological systems central to DOE's mission. This includes characterizing living biological systems in physiological conditions, ideally, inside living tissues and biofilms and other microbial communities. To that end, we are pursuing an integrated three-part research and development strategy focusing on hardware, algorithms, and chemistry. Our biological test systems are (1) lignocellulose degradation and (2) low-dose biology.

The termite hindgut microbial community is one of nature's most efficient lignocellulose biodegrading "factories". Metagenomic analysis has revealed that the hindgut microbial community is composed of about two hundred microbial species. We seek a fundamental understanding of how community spatial organization and composition affects lignocellulose degradation using 2D wide-field transmission electron microscopy (TEM) as well as 3D focused ion beam scanning electron microscopy (FIB/SEM) imaging. Structural analysis is complemented by $16 \mathrm{~S}$ rRNA fluorescence in-situ hybridization (FISH) labeling and correlative light and electron microscopy.

The SatB1 protein is a chromatin organizer that regulates genes by looping chromatin. SatB1 is a central participant in the cellular response to low-dose ionizing radiation and, like many other factors involved in DNA repair, also has been implicated in human diseases such as breast cancer. SatB1 is currently the only protein known to organize the genome by tethering its target genes onto its cage structure. Once genes are recruited to the SatB1 cage, it serves as a platform for assembly of chromatin remodeling/modifying factors, which in turn establishes the specific epigenetic status of these loci.

\section{Accomplishments:}

For the termite hindgut project, we have extensively characterized in $2 \mathrm{D}$ and $3 \mathrm{D}$ the $3 \mathrm{D}$ organization of the termite hindgut lignocellulolytic community members, as well their respective strategies for cell wall-adhesion, and lignocellulosic deconstruction, by employing the recently developed Focused Ion Beam Scanning Electron Microscopy (FIB/SEM), which allows the 3D reconstruction of large volumes. 3D ultrastructural quantitative analysis were complemented by CARD-FISH labeling of resin-embedded sections, using species specific 16S rRNA probes. While a multitude of community members is capable for lignocellulose degradation, we found one type of microbe to enzymatically drills narrow channels into the plant biomass cell wall, without the apparent need for other community members. We have identified this unknown "super-degrader" microbe and are currently studying its protein inventory. Enzymes secreted from such microbes may hold the key for effective commercial lignocellulosic biofuel production, and may serve as a posterchild for targeted bioprospecting.

For the SatB1 protein studies, by simultaneously refining imaging hardware (reducing thermal drift), algorithms (to generate 3D nanometer precision maps), and chemistry (sample fixation protocols), we have succeeded in generating the first-ever nanometer precision maps of how SatB1 is organized in the mammalian nucleus, a critical requirement if we are to understand its ability to function as a 'master regulator' of gene expression. In parallel work, we have also developed a strategy for reliable and specific delivery of materials to the mammalian nucleus via the nuclear pore complex, which allows us to follow proteins as they move inside the nucleus and bind DNA. Together, the ability to create nanometer precision maps of DNA binding proteins, as well as watch complexes assemble and move inside the nucleus, will greatly facilitate DOE's overall goal of a mechanistic understanding of DNA repair and low-dose radiation biology. 
Predictive High-Throughput Assembly of Synthetic Biological Systems: From Gene Expression to Carbon Sequestration

Principal Investigators: Cheryl Kerfeld, Adam Arkin, Eddy Rubin

\section{Project Description}

The goal of the project is to develop methods for the design and assembly of a type of bacterial organelle, the carboxysome, with increased $\mathrm{CO} 2$ fixation activity. The project is divided into two research components: the Arkin group will design and characterize controllers for gene expression, while the Kerfeld group will assemble and test various combinations of carboxysome structural components for assembly and function in cyanobacteria. The results of these studies will be combined to enable a predictive approach to design of functionally enhanced carboxysomes for specific environmental conditions. The carboxysome is the best characterized example of a bacterial microcompartment; these are naturally occurring, selfassembling organelles composed entirely of protein that confer distinctive functions on the organisms that encode them. Bacterial microcompartments are anticipated to have widespread use in synthetic biology-based approaches to engineering bacteria for the production of useful compounds. Accordingly, the methods developed for and the results of this project will be useful in the design and characterization of other types of bacterial microcompartments that can be used as metabolic modules.

\section{Accomplishments}

In the first year we have obtained cyanobacterial carboxysome mutants, characterized their ultrastructure and physiology and established methods for their genetic manipulation. This has involved assembling the infrastructure for growth and manipulation of these organisms, creating plasmids designed to integrate a variety of carboxysome genes under different promoters. These have been tested by rescue experiments of the different mutants. We have also initiated experiments to effectively mimic gene duplication and horizontal gene transfer into wild-type cyanobacteria. Experiments are ongoing to evaluate the effects of overexpression of these expansions of the carboxysome gene complement on growth under both normal conditions and conditions of stress. Development of these strains and tools will substantially enhance the genetic toolbox available for use with cyanobacteria, thus facilitating future synthetic biology experiments aimed at expanding metabolic capabilities within this important bacterial phylum.

We have also pioneered a number of gene expression control systems that will allow the tuning of expression of multiprotein complexes such as the carboxysome generically across organisms. We believe that these will be useful in exploring the stoichiometric constraints on carboxysome formation and function and in testing the assembly of differently composed carboxysomes. These controllers do not depend on any other host factors and should thus be readily transferrable into cyanobacteria easily. We have also successfully developed a single molecule screening procedure for measuring expression of transcripts. 
Multifunctional Window Coatings for High-Performance Buildings

Principal Investigators: Delia Milliron, Jeffrey Urban, Yuegang Zhang, André Anders, Thomas Richardson

Project Description

This project aims to develop window coatings capable of greatly improving building energy performance. Specifically, we are developing materials to support two key technological advancements (1) coatings which harvest light outside the visible spectrum to produce electrical power and (2) spectrally selective dynamic window coatings. While the best warm climate coatings currently available simply reflect near infrared light (NIR), we aim to harness this massive energy source. Window coatings with switchable NIR transmittance would permit active control in response to temporal and seasonal changes in climate.

One major component of the project focuses on the thin films of transparent conducting oxide (TCO) material which provide current to switchable windows or harvest power from inwindow energy conversion devices. We are developing advanced physical vapor deposition methods involving plasma processing to improve NIR transparency while keeping conductivity high and costs low. A second major component involves the development nanocrystal-based electrochromic switching films which modulate NIR transmittance under an applied potential.

\section{Accomplishments}

In developing innovative TCO materials, we have focused on aluminum-doped zinc oxide (AZO) because the material cost is far lower than the standard tin-doped indium oxide (ITO) or other alternatives. Typical carrier mobilities in AZO are $20-30 \mathrm{~cm}^{2} / \mathrm{V}$-s, a value which makes the simultaneous realize of high conductivity and good visible and NIR transparency impossible. In our work, AZO films with varying dopant concentrations have been investigated using various deposition technologies, including traditional magnetron sputtering and the cathodic arc plasma deposition, a new method under active development at LBNL. The latter operates at much lower process voltage and thereby limits damage from high energy ions impacting the film during growth. As a result, highly transparent, indium-free films with mobilities between 40 and 50 $\mathrm{cm}^{2} / \mathrm{Vs}$ have been obtained. We will further optimize the processing conditions to improve this result. Then, we intend to focus on layering strategies such as buffer layers or modulation doping to achieve even higher mobilities.

For the development of NIR-modulating electrochromics, we have synthesized a variety of colloidal nanocrystals of TCO materials. These include different sizes (from 5-20 nm) of ITO and AZO nanocrystals with doping levels from 1 to $8 \%$. In each case, the nanocrystals have been characterized by electron microscopy, x-ray diffraction, elemental analysis, and optical spectroscopy.

To prepare electrochromic films, the nanocrystals have been deposited from solvent dispersions using spin coating or drop casting. After deposition, post processing is necessary to remove the ligands that coat the nanocrystals, placing them in electrical FRQMFWZ IUKHFFKRLXHUD

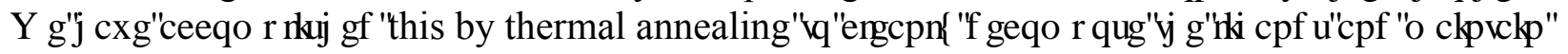
the conductive nature of the TCO. Efforts are underway to use FKHP IFDOFRQNRORILAOU DOQG to streamline the post-processing conditions. The resulting TCO nanocrystal films are conductive and also exhibit modulation of NIR transmittance between $\sim 25 \%$ and $\sim 75 \%$ in initial observations. The relationship between modulation of characteristics of the nanocrystals (size and composition) will be investigated systematically. 


\title{
Coupled Process Models, Separations, Licensing/Monitor Advanced Nuclear Fuel Cycles
}

\author{
Principal Investigator(s): Andrew Canning, Phil Collela, Per Peterson, \\ Lee Schroeder, Jim Siegrist, David Shuh, Niels Jensen (UC Davis)
}

\section{Project Description:}

Advanced nuclear fuel cycles require enhanced computer modeling and performance monitoring tools to optimize the fuel cycle, for reactor design and licensing and for associated reprocessing and waste related issues. This project is a coordinated effort to develop next-generation simulation modeling tools for reactor physics and licensing, and modeling fuel cycle actinide behavior in waste forms.

\section{Accomplishments:}

The work on extending Adaptive Mesh Refinement methods succeeded in the implementation of the full conjugate heat transfer (solids and liquids) solver for a buoyant fluid in complex geometries (expected for use in future fast reactors) using 100 's of processors on Hopper and the Cray XT5 at NERSC. In addition, convective and conjugate heat transfer solvers were successfully validated with demonstrated scaling of the conjugate heat transfer to 100's of processors--important for fluid flow problems in future reactors.

In FY10 work related to identifying and developing advanced simulation methods for reactor and nuclear facility applications focused on analyzing the dynamics of pebble bed reactors. Discrete element methods are the best approach to confidently model pebble bed reactor dynamics in core geometries, but are still too computationally intensive to be used for multiple design iterations. Significant effort was devoted to developing simplified models informed by strategic use of high performance computational results, demonstrating that it is possible to accurately match experimental data from these reactors while achieving a significant reduction in computational time.

Electronic structure of f-element complexes and materials relevant to nuclear energy were characterized at the Advanced Light Source by near-edge x-ray absorption fine structure (NEXAFS) of the light element K-edges. Complementing the NEXAFS measurements probing unoccupied states, soft x-ray emission spectroscopy (XES) studies were used to gain information on the unoccupied states. Results of the combined experimental studies are ideal for comparison with theory. Focus of the soft $\mathrm{x}$-ray studies was on bonding in f-element metal complexes (La, Nd, Eu and Gd) with several sets of novel ligands containing nitrogen atoms as analogs for the corresponding actinide complexes. Theoretical work focused on $\mathrm{f}$ electron bonding in various uranium complexes, and proposed Pu complexes with new ligands. These calculations provided a basis for understanding the experimental results and advance guidance for selecting appropriate ligand sets for the separation of trivalent actinides from lanthanides in advanced fuel cycles. 



\section{Publications List}

AFRD-Anders LB10032 Plasma-Assisted High Rate Deposition Concept for Energy

Applications

Publications \& talks

A. Anders, "Physics of high power impulse magnetron sputtering," Plenary Lecture presented at the Twelfth Int. Conf. on Plasma Surface Engineering (PSE), GarmischPartenkirchen, Germany, 2010.

A. Anders, manuscript based on the above lecture, submitted to Surface and Coatings Technology, under review.

A. Anders, J. Brown, S. Lim, R. Mendelsberg, L. Gontijo, K. M. Yu, M. Field; "Improving aluminum doped zinc oxide films by minimizing negative ion damage," Invited Talk presented at the 8th Int. Conf. Coatings on Glass and Plastics, Braunschweig, Germany, 2010.

A. Anders and J. Brown, "A plasma lens for magnetron sputtering," submitted to IEEE Trans. Plasma Sci., under review.

\section{Invention Disclosures}

A. Anders, invention disclosure IB-2803: "Method and Apparatus for Sputtering with a Plasma Lens," filed December 7, 2009.

A. Anders, invention disclosure IB-2830: "Plasma lens with hot electrode." filed January 7 , 2010

\section{AFRD-Ludewigt, Seidl LB10012 Ion Beam Driven Fission Hybrids}

P.A. Seidl, "Fusion-Fission Hybrids Driven By Heavy Ion Inertial Fusion," contributed paper to the Fusion-Fission Hybrid Workshop, Gaithersburg, MD, Sept. 30 - Oct. 2, 2009. http://web.mit.edu/fusion-fission/

C. Di Sanzo, J. Cohen, T. Cisneros, B. Ludewigt, E. Greenspan; "An Accelerator Driven Energy Multiplier for Doubling Uranium Ore Utilization," abstract submitted to AccApp '11 - Tenth International Topical Meeting on Nuclear Applications of Accelerators Knoxville, TN. April 3-7, 2011, full paper being drafted

AFRD-Robin LB10031 Novel Accelerator and Engineering Strategies for Ion Beam Cancer Therapy

S. Caspi, D. Robin, W. Wan, A. Sessler, M. Yoon, C. Sun; "Compact, light weight, superconducting gantry for Carbon Ion Beam Therapy," draft for submission possibly to Nucl. Instr. and Methods.

D. Robin, "Gantries," invited talk given at the "The 13th workshop on Ion Beams in Biology and Medicine," Berkeley, CA, USA 26-29 October, 2010

D. Robin, "An Overview of Next Generation Gantries" invited talk to be given at the "2nd Workshop on Hadron beam Therapy of Cancer," Erice, Sicily, Italy, May 20, 2011 - May 27, 2011

S. Caspi, "A SC Dipole Magnet for a Carbon Therapy Gantry," invited talk to be given at the "2nd Workshop on Hadron beam Therapy of Cancer", Erice, Sicily, Italy, May 20, 2011 May 27, 2011 
AFRD-Sannibale LB09007 Experimental Accelerator R\&D Toward a Future Light Source

F. Sannibale, B. Bailey, K. Baptiste, et al; "Status of the LBNL normal-conducting CW VHF electron photo-gun," Proceedings of the 2010 Free Electron Laser Conference, Malmo, Sweden, August 2010.

C. F. Papadopoulos, J. Corlett, G. Penn, J. Qiang, F. Sannibale, J. Staples, M. Venturini; "Multiobjective optimization for the Advanced Photoinjector Experiment (APEX)," Proceedings of the 2010 Free Electron Laser Conference, Malmo, Sweden, August 2010.

F. Sannibale, "A normal conductive RF photo-injector for high repetition rate X-ray free electron lasers," seminar June 29, 2010, Cornell University - Ithaca, NY.

F. Sannibale, "A normal conductive RF photo-injector for high repetition rate X-ray free electron lasers," seminar February 8, 2010, DESY - Zeuthen Seminar, Berlin, Germany.

AFRD-Schenkel LB08017 Quantum Information Science with Integrated Color Centers in Diamond

D.M. Toyli, C.D. Weis, G.D. Fuchs, T. Schenkel, D.D. Awschalom; “Chip-scale nanofabrication of single spins and spin arrays in diamond," NanoLetters 10, 3168 (2010). url: http://pubs.acs.org/doi/pdf/10.1021/nl102066q

G. Fuchs, V. Dobrovitski, D. Toyli , J. Heremans, C. Weis, T. Schenkel, D.D. Awschalom; "Excited-state spin coherence of a single nitrogen-vacancy centre in diamond," Nature Physics 6, 668 (2010). url: http://www.nature.com/nphys/journal/v6/n9/pdf/nphys1716.pdf

AFRD-Toth LB08022 Experimental Demonstration of a Laser-Plasma-Accelerator Driven Free-Electron Laser

\section{Refereed Publications}

A.J. Gonsalves, K. Nakamura, C. Lin, et al; "Plasma Channel Diagnostic Based on Laser Centroid Oscillations," Physics of Plasmas 17, 056706 (2010).

D. Panasenko, A.J. Shu, A. Gonsalves, et al; "Demonstration of a plasma mirror based on a laminar flow water film," J. of Appl. Phys. 108, 044913 (2010).

M.S. Bakeman, J. van Tilborg, T. Sokollik, et al; "Calibration of a microchannel plate based extreme ultraviolet grazing incident spectrometer at the Advanced Light Source," Rev. Sci. Instrum. 81, 10E313 (2010)

\section{Conference Proceedings}

M.S. Bakeman, W.M. Fawley, W. Leemans, K. Nakamura, K.E. Robinson, C.B. Schroeder, C. Toth; "Undulator-Based Laser Wakefield Accelerator Electron Beam Diagnostic," in PAC 2009 (www.jacow.org) - LBNL-2355E.

D. Panasenko, A. J. Gonsalves, W. Leemans, K. Nakamura, C. B. Schroeder, A. Shu, Cs. Toth; "Development of Water Jet Plasma Mirror for Staging of Laser Plasma Accelerators," in PAC 2009 (www.jacow.org).

J. Osterhoff, T. Sokollik, K. Nakamura, et al; "Transport and Non-Invasive Position Detection of Electron Beams from Laser-Plasma Accelerators," AIP Conf. Proc. 1299, 575-579 (2010)

N. H. Matlis, M. Bakeman, C. G. R. Geddes, et al; "Ultrafast Diagnostics for Electron Beams from Laser Plasma Accelerators," AIP Conf. Proc. 1299, 43-51 (2010)

T. Sokollik, S. Shiraishi, J. Osterhoff, E. Evans, A.J. Gonsalves, K. Nakamura, J. van Tilborg, C. Lin; “Tape-Drive Based Plasma Mirror,” AIP Conf. Proc. 1299, 233-237 (2010) 
A.J. Gonsalves, K. Nakamura, C. Lin, et al; "Plasma Channel Diagnostic Based on Laser Centroid Oscillations," AIP Conf. Proc. 1299, 150-155 (2010)

M.S. Bakeman, J. Van Tilborg, K. Nakamura, et al; "Undulator-Based Laser Wakefield Accelerator Electron Beam Energy Spread and Emittance Diagnostic," AIP Conf. Proc. 1299, 133-138 (2010)

\section{Invited Talks}

W.P. Leemans, "Overview of LWFA Experiments," The Physics \& Application of High Brightness Electron Beams (HBEB 2009), November 2009.

W.P. Leemans, "Advances in Laser/Plasma Based Sources at LBNL," FLS 2010, ICFA Beam Dynamics Workshop, SLAC, March 3, 2010.

W.P. Leemans, "The BErkeley Lab Laser Accelerator (BELLA): A $10 \mathrm{GeV}$ Laser Plasma Accelerator," Plenary Talk at AAC2010 Workshop, Annapolis, MD, June 2010.

W.P. Leemans, "Perspectives of High Power Laser Technology for Particle Beam Acceleration," 46th ICFA Advanced Beam Dynamics Workshop on High-Intensity and High-Brightness Hadron Beams (HB 2010), Sept. 28, 2010, Morschach, Switzerland

W.P. Leemans, "Laser Plasma Accelerators: Concepts, Program, and Dreams," FiO/Laser Sciences Workshop, October 27, 2010.

C.B. Schroeder, "Frontiers of Laser Plasma Acceleration," Towards a 5th Generation Light Source Workshop, Avalon, CA, Oct. 2010.

C.B. Schroeder, "Prospects for a Table-top FEL," ICFA workshop on The Physics and Applications of High Brightness Electron Beams 2009, in Maui, HI, Nov. 2009.

Cs. Toth, "Optical Accelerators and the BELLA Project," UC Berkeley, , March 12, 2010.

Cs. Toth, "Radiation Sources Based on Laser Plasma Accelerators," ECLIM - 2010, Budapest, Hungary, September 8, 2010.

Cs. Toth, "Laser Plasma Acceleration Experiments with a 100-TW Class Laser and Lessons Learned," ICUIL - 2010, Watkins Glen, NY, USA, September 27, 2010.

AFRD-Vay LB08023 Lorentz Compaction of Scales for Ultra-efficient Simulation of Advanced Accelerators (and other systems)

J.-L. Vay, C.G.R. Geddes, E. Cormier-Michel, D.P. Grote; "Effects of hyperbolic rotation in minkowski space on the modeling of plasma accelerators in a lorentz boosted frame," to be submitted to Phys. Plasmas. arXiv:1011.0917v2, url: http://arxiv.org/abs/1011.0917

J.-L. Vay, C.G.R. Geddes, E. Cormier-Michel, D.P. Grote; "Efficient modeling laser wakefield accelerators in a lorentz boosted frame," to be submitted to Phys. Plasmas.

J.-L. Vay, C.G.R. Geddes, E. Cormier-Michel, D.P. Grote; "Modeling laser wakefield accelerators in a lorentz boosted frame," arXiv:1009.2727v2. url: http://arxiv.org/abs/1009.2727

J.-L. Vay, C.G.R. Geddes, E. Cormier-Michel, D.P. Grote; "Modeling laser wakefield accelerators in a lorentz boosted frame," Proc. 14th Advanced Accelerator Concepts Workshop, Annapolis, MD (2010).

W.P. Leemans, R. Duarte, E. Esarey, et al; "The BErkeley Lab Laser Accelerator (BELLA): a $10 \mathrm{GeV}$ laser plasma accelerator," Proc. 14th Advanced Accelerator Concepts Workshop, Annapolis, MD (2010).

J.-L. Vay, C.G.R. Geddes, E. Cormier-Michel, D.P. Grote; "Numerical methods for instability mitigation in the modeling of laser wakefield accelerators in a lorentz boosted frame," submitted to J. Comput. Phys. 
W. Fawley, J.-L. Vay; "Full electromagnetic FEL simulation via the Lorentz-boosted frame transformation," Proc. $32^{\text {nd }}$ International Free Electron Laser Conference, Sweden (2010), paper MOPB01

W. M. Fawley, J.-L. Vay; "Full electromagnetic simulation of coherent synchrotron radiation via the Lorentz-boosted frame approach," Proc. $1^{\text {st }}$ International Particle Accelerator Conference, Kyoto (2010). url: http://accelconf.web.cern.ch/AccelConf/IPAC10/papers/tupec064.pdf

AFRD-Venturini, Wurtele LB10024 Linac Driver and Coherent Soft X-ray Sources

A. Zholents and G. Penn, "Obtaining two Attosecond Pulses for X-ray Stimulated Raman Spectroscopy," Nucl. Instr. and Methods in Phys. Res. A 612, 254 (2010).

J. Qiang, "Short Wavelength Seeding through Compression for free electron lasers," Nucl. Instr. \& Methods in Phys. Res. A 621, 39 (2010).

M. Venturini, M. Migliorati, C. Ronsivalle, M. Ferrario, C. Vaccarezza; "Dynamics of Longitudinal Phase-Space Modulations in an rf Compressor for Electron Beams," Phys. Rev. ST Accel. Beams 13, 080703 (2010).

W. Barletta et al, "Free Electron Lasers: Present Status and Future Challenges, "Nucl. Instr. and Methods in Phys. Res, A 618, 69 (2010).

J. Wurtele, P. Gandhi, X-W Gu, et al; "Tunable Soft X-Ray FEL Oscillators," The 32nd International Free Electron Laser Conference FEL 2010, Paper ID: TUOCI2 (2010).

S. Di Mitri, M. Cornacchia, P. Craievich G. Penco, S. Spampinati, M. Venturini, A. Zholents; "Comparative Study of the Fermi@Elettra Linac with one and two-stage Electron Bunch Compression," The 32nd International Free Electron Laser Conference FEL 2010, THPB03 (2010).

M. Venturini, "Evolving Beam Charge Density in Chicanes Following Interaction with Lasers," CBP Technical Note, CBP-TN-409, Berkeley (2010).

D. Xiang et al, "Preliminary results of the echo-seeding experiment ECHO-7at SLAC," Proceedings of IPAC 2010, paper TUPE072.

M. Dunning et al, "A proof-of-principle echo-enabled harmonic generation experiment at SLAC," Proceedings of IPAC 2010, Paper TUPE069.

J. Corlett, et al, "Design Studies for a Next Generation Light Source Facility at LBNL," The 32nd International Free Electron Laser Conference FEL 2010, paper: MOPA06

J. Corlett, et al, "Design Studies for a VUV-Soft X-ray FEL Facility at LBNL," Proceedings of International Part. Acc. Conference, 2639, Kyoto Japan (2010).

N. Thompson, "Study of an HHG-Seeded Free-Electron Laser for the LBNL Next Generation Light Source," draft LBNL Technical Report (2010).

M. Venturini, "Microbunching from a Beam with an Energy Spread Distributed Inhomogeneously in the Transverse Plane," CBP Technical Note, CBP-TN-408, Berkeley (2010)

ALS-Hexemer et al LB08009 Soft X-ray Scattering as a New Probe of Polymer Systems

H. Yan, S. Swaraj, C. Wang, et al; "Influence of Annealing and Interfacial Roughness on the Performance of Bilayer Donor/Accept or Polymer Photovoltaic Devices," Advanced Functional Materials (in press 2010)

C. Wang, J. Nasiatka, E. Chan, et al; "Resonant Soft X-ray Scattering of Polymers with a 2D Detector: Initial Results and System Developments at the Advanced Light Source," J. of Physics: Conference Series (in press 2010) 
H. Ade, C. Wang, H. Yan; "The Case for Soft X-rays: Improved Compositional Contrast for the Structure and Morphology Determination with Real and Reciprocal Space Methods," J. of Physics: Conference Series (in press 2010)

S. Swaraj, C. Wang, H. Yan, et al; "Nanomorphology of Bulk Heterojunction Photovoltaic Thin Films Probed with Resonant Soft X-ray Scattering," Nano Letters 10 (8), 28632869.

B. J. Rancatore et al; "Nanostructured Organic Semiconductors via Directed Supramolecular Assembly," Acs Nano, 4 (5), 2721-2729.

ALS-Hexemer LB10009 Long-Range Ordering of Block Copolymers on Patterned Silicon

L. Dong Hyun; P. Soojin; G. Weiyin; T.P. Russell; "Highly Ordered Nanoporous Template from Triblock Copolymer," submitted to ACS Nano.

L. Dong Hyun, X. Ting; H. Sung Woo, A. Hexemer; H.A., Padmore, T.P. Russell; "SelfAssembly of Block Copolymers on Well-modulated Sawtooth Surface," presentation at American Physical Society (APS) March Meeting (March, 2010).

E.R. Chan, L. Dong Hyun, D.L. Voronov; H.A. Padmore; X. Ting, T.P. Russell; A. Hexemer; "Self-Assembly of Block Copolymers on Well-modulated Sawtooth Surface," presentation at American Physical Society (APS) March Meeting (March, 2010).

H. Sung Woo; H. June, L. Dong Hyun, X. Ting, J. Won Ho, P. Soojin, T.P. Russell; "Highly Oriented and Aligned Line Patterns of Block Copolymer over macroscopic Area, submitted to Science.

L. Dong Hyun, X. Ting; H. Sung Woo, A. Hexemer; H.A., Padmore, T.P. Russell; "SelfAssembly of Block Copolymers on Well-modulated Sawtooth Surface," draft TBD.

ALS-Kilcoyne LB10034 Search for a Permanent Electron Electric Dipole Moment (EDM)

J.G. Kalnins, "Electrostatic Lens End-Field Defocusing in a Neutral Cesium Atom Fountain," draft to be submitted to Physical Review Special Topics - Accelerators and Beams.

ALS-Kunz LB10011 Structure Solution of Inorganic Materials Using Energy Resolved Laue Microdiffraction

C. Dejoie, M. Kunz, N. Tamura, C. Bousige, K. Chen, S. Teat, C. Beavers, C. Baerlocher; "Determining the Energy Dependant X-ray Flux Variation of a Synchrotron Beamline Using Laue Diffraction Patterns," accepted in J. Appl. Cryst.

C. Dejoie, M. Kunz, N. Tamura, et al; "Towards in-situ Monitoring of Structural Changes in Inorganic Materials by Synchrotron Laue X-ray Microdiffraction," oral presentation at MRS Fall Meeting, 2010

K. Chen, C. Dejoie, M. Kunz, N. Tamura; „Identification of Dauphine Twins in Quartz using Laue Microdiffraction," draft for submission to Earth and Planetary Letters.

C. Dejoie, M. Kunz N. Tamura, C. Beavers, S. Teat, L. McCusker, C. Baerlocher; "Structure Solution of Zeolite Systems using Laue Microdiffraction," draft for submission to $J$. of Appl. Crystallography.

ALS-Marchesini LB10014 Nanoscale Surveyor

F. Maia, A. MacDowell, S. Marchesini, et al; "Compressive phase contrast tomography," in Image Reconstruction from Incomplete Data VI, edited by Philip J. Bones, Michael A. Fiddy, Rick P. Millane). Proc. SPIE Vol. 7800, 78000F (2010). [arxiv:1009.1380] 
(various,) "Diffraction Microscopy with COSMIC at the Advanced Light Source," XRM2010: 10th International Conference on X-Ray Microscopy, Aug. 15-20, 2010, Chicago, IL.

ALS-Padmore LB09036 High Quantum Yield Multi-Alkali Cathodes for psec Pulsed Electron Sources

D.H. Dowell, I. Bazarov, B. Dunham, et al; "Cathode R\&D for future light sources," Nucl. Instrum. Meth. A, A622, 85-697.

ALS-Warwick LB10026 Test Monochromator/ Spectrometer Systems with Prototype High Density Gratings for High Resolution X-ray Scattering

D. L. Voronov, M. Ahn, E.H. Anderson, et al; "High Efficiency 5000 lines/mm Multilayer Coated Blazed Gratings for Extreme Ultraviolet Wavelengths," Opt. Lett 35, 2615 (2010).

T. Warwick, H.A.Padmore, D. Voronov, V. Yashchuk; "A Soft X-ray Spectrometer using a Highly Dispersive Multilayer Grating," Proc. 10th Intl. Conf. on Synch. Radn. Instrum., R. Garret, I. Gentle, K. Nugent and S. Wilkins, American Institute of Physics, p 760-780.

ALS-Yashcuk LB09030 X-Ray Optical Metrology for Coherence-Preserving Adaptive Optics

S. Yuan, M. Church, V.V. Yashchuk, et al; "Elliptically Bent X-ray Mirrors with Active Temperature Stabilization," X-Ray Optics and Instrumentation 784732/1-9 (2010).

S. Yuan, M. Church, V.V. Yashchuk, et al; "At-Wavelength Optical Metrology Development at the ALS," Proc. of SPIE 7801, 78010D/1-12 (2010).

S. Yuan, V.V. Yashchuk, K.A. Goldberg, et al; "Development of in situ, at-wavelength metrology for soft x-ray nano-focusing," Nucl. Instr. and Meth. A, in press. doi:10.1016/j.nima.2010.10.134.

S. Yuan, K.A Goldberg, V.V. Yashchuk, et al; "Cross-check of ex-situ and in-situ metrology of a bendable temperature stabilized KB mirror," Nucl. Instr. and Meth. A, in press. doi:10.1016/j.nima.2010.09.120.

S. Yuan, V.V. Yashchuk, K. A. Goldberg, et al; "Surface Slope Metrology on Deformable Soft X-ray Mirrors," The Tenth International Conference on Synchrotron Radiation Instrumentation (Melbourne, Victoria, Australia. September 27 -- October 2, 2009), AIP Conf. Proc. 1234, 789-92 (2010).

S. Yuan, K.A Goldberg, V.V. Yashchuk, et al; "At-wavelength and optical metrology of bendable x-ray optics for nanofocusing at the ALS," Digest of the OSA Optics and Photonics Congress: Frontiers in Optics 2009, Laser Science XXV, Special Symposium on Optics for Imaging at the Nanoscale and Beyond; San Jose, California, USA, October $11-15,2009$.

CH-Budker, Mueller LB10002 Direct Comb Spectroscopy of Lithium in the Vacuum Ultraviolet and Beyond

H. Müller, "Precision tests of General Relativity with Matter Waves," Proc. of the International Conference on Atomic Physics, 2010, in press.

H. Müller, "Precision Tests of General Relativity using Matter Wave Interferometry," invited talk at Cornell University Physics Colloquium Oct. 25, 2010.

H. Müller, "Gravitational Redshift, Equivalence Principle, and Matter Waves," invited talk at NASA ISS Workshop on Fundamental Physics, Oct 13-15, 2010. 
H. Müller, "Precision tests of General Relativity with Matter Waves," invited talk at Harvard-MIT Center for Ultracold Atoms, Oct. 26, 2010

H. Müller, "Precision tests of General Relativity with Matter waves," invited talk at San Francisco State University Physics Colloquium, Nov 8, 2010.

H. Müller, "Gravitational Redshift, Equivalence Principle, and Matter Waves," invited talk at University of Kentucky Physics Colloquium, Oct. 1, 2010.

H. Müller, "Gravitational Redshift, Equivalence Principle, and Matter Waves," invited talk at UC Berkeley AMO Seminar, Aug. 1, 2010.

H. Müller, "Precision Tests of General Relativity using Matter wave Interferometry," invited talk at CPT and Lorentz Symmetry, Bloomington, Indiana, 2010.

H. Müller, invited talk at Annual meeting of the Department of Atomic, Molecular and Optical Physics of the American Physical Society, Houston, TX, May 27, 2010.

G. Kim, "A precision test of the Equivalence Principle using Lithium-6 and 7 interferometers," invited talk at Annual meeting of the Department of Atomic, Molecular and Optical Physics of the American Physical Society, Houston, TX, May 2010.

H. Müller, invited talk at Wilhelm and Else Heraeus School on Matter Wave Interferometers, Bad Honneff, Germany, June 14, 2010.

H. Müller, "A precision measurement of the gravitational redshift and other applications of matter waves," invited talk at International Conference on Atomic Physics (ICAP), Cairns, Australia. 2010.

H. Müller, "A Precision Measurement of the Gravitational Redshift and Other Applications of Atom Interferometers," invited talk at Physics Colloquium, UC Santa Cruz, May 20, 2010.

CH-Gessner LB08008 Probing Transient Molecular Entanglement Using Femtosecond High Resolution Delayed-field Coincidence Imaging

O. Kornilov, R. Wilcox, O. Gessner; "Nanograting-Based Compact Vacuum Ultraviolet Spectrometer and Beam Profiler for In Situ Characterization of High-Order Harmonic Generation Light Sources," Rev. Sci. Instrum. 81, 063109 (2010). url: http://rsi.aip.org/rsinak/v81/i6/p063109_s1.

CH-Harris LB09011 Dynamics of Homogeneous Catalysis Reactions Investigated with Transient Two-Dimensional Infrared Spectroscopy on the Pico- to Microsecond Timescale

K. Sawyer, J. Cahoon, J. Shanoski, et al; "Time-resolved IR studies on the mechanism for the functionalization of primary $\mathrm{C}-\mathrm{H}$ bonds by $\mathrm{Cp} * \mathrm{~W}(\mathrm{CO}) 3$ (Bpin)," J. Am. Chem. Soc. 2010, 132, 1848-1859.

CH-Lukens LB10037 Quantifying f-electron Exchange Coupling in Actinide and Lanthanide Complexes

W. Lukens, M. Walter; "Quantifying Exchange Coupling in f-Ion Pairs Using the Diamagnetic Substitution Method," Inorg. Chem. 49, 4458-4465 (2010). url: http://pubs.acs.org/doi/abs/10.1021/ic100120d?journalCode=inocaj\&quickLinkVolume= $\underline{49 \& \text { quickLinkPage }=4458 \& \text { volume }=49}$

CS-Agarwal LB10001 Enabling High Performance Computing (HPC) Workflows on Clouds

K. Jackson, L. Ramakrishnan, K. Runge, R. Thomas; "Seeking Supernovae in the Clouds: A Performance Study," ACM Science Cloud 2010, Chicago IL, June 21, 2010. 
K. Jackson, K. Muriki, L. Ramakrishnan, K. Runge, R. Thomas; "Performance and Cost Analysis of The Supernova Factory on The Amazon AWS Cloud," submitted to Wiley Journal of Scientific Programming.

CS-Carter LB08035 Enhancing the Effectiveness of Manycore Chip Technologies for HighEnd Computing

K. Madduri, E.-J. Im, K. Ibrahim, S. Williams, S. Ethier, L. Oliker; "Gyrokinetic Particle-inCell Optimization on Emerging Multi- and Manycore Platforms," submitted to Parallel Computing Journal.

CS-Rotem LB08015 Energy-Smart Disk-Based Mass Storage System

E.J. Otoo, D. Rotem, S.-C. Tsao: "Dynamic Data Reorganization for Energy Savings in Disk Storage Systems," Scientific and Statistical Database Management Conference 2010: 322-341.

J. Kim, D. Rotem; "Using Replication for Energy Conservation in RAID Systems," International Conference on Parallel and Distributed Processing Techniques and Applications 2010, 703-709 82.

J. Chou, J. Kim, D. Rotem; Energy-Aware Scheduling in Disk Storage Systems," submitted for publication in International Conference on Distributed Computing Systems.

J. Kim, D. Rotem; "Energy Proportionality for Disk Storage Using Replication," submitted for publication in 14th International Conference on Extending Database Technology.

J. Chou, J. Kim, D. Rotem; "Energy Saving Techniques for Disk Storage Systems," book Chapter in Handbook of Energy-Aware and Green Computing.

CS-Sethian LB10020 Transforming Data: From Images to Models to Computational Input

R. Saye, J.A. Sethian; "Tracking Complex Interfaces: Foams and Membranes," in preparation for submission to J. of Computational Physics.

R. Rycroft, R. Saye, J.A. Sethian; "PDE-based techniques for computing elastic, hydrodynamic, and geometric forces in cell growth and stability," in preparation for submission to $J$. of Computational Physics.

D. Ushizima, "PDE-based techniques for extracting cell boundaries from time-varying images," in preparation for submission to J. of Math. Imaging and Vision.

CS-Shalf et al LB08002 Holistic Approach to Energy Efficient Computing Architecture

G. Hendry, J. Chan, S. Kamil, L. Oliker, J. Shalf, L. Carloni, K. Bergman; "Silicon Nanophotonic Network-On-Chip using TDM Arbitration," IEEE Hot Interconnects Conference (HOTI), 2010.

S. Kamil, L. Oliker, A. Pinar, J. Shalf; "Communication Requirements and Interconnect Optimization for High-End Scientific Applications," IEEE Trans. Parallel Distrib. Syst. 21(2): 188-202 (2010).

J. Krueger, D. Donofrio, J. Shalf, S. Williams, L. Oliker, F. Pfreundt; "Hardware Software Co-Design for Seismic Modeling Applications using Higher-Order Stencils," submitted to IPDPS 2011.

CS-Strohmaier LB08036 Reference Benchmarks for the Dwarfs (Algorithms)

A. Kaiser, S. Williams, K. Madduri, K. Ibrahim, D. H. Bailey, J. W. Demmel, E. Strohmaier; "A Case for a Testbed of Kernels for Software/Hardware Co-Design Research," HotPar '10: Second USENIX Workshop on Hot Topics in Parallelism, Berkeley, June 2010. 
E. Strohmaier. S. Williams, A. Kaiser, K. Madduri, K. Ibrahim, D. H. Bailey, J. W. Demmel; "A Kernel Testbed for Parallel Architecture, Language, and Performance Research," ICNAAM2010 and AIP Conference Proceedings 1281, 1, September 2010, 1297-1300.

A. Kaiser, S. Williams, K. Madduri, K. Ibrahim, D. H. Bailey, J. W. Demmel, E. Strohmaier: "TORCH Computational Reference Kernels, A Testbed for Computer Science Research," Technical Report: $L B N L$ and UCB/EECS-2010-144, December 2010.

CS-Wu LB09028 Managing Petascale Data with Emerging Computer Architectures

K. Wu, K. Madduri, S. Canon; "Multi-level bitmap indexes for flash memory storage," Proc. 14th ACM International Database Engineering and Applications Symposium (IDEAS '10), LBNL Technical Report \# pending (August 2010). url: http://dx.doi.org/10.1145/1866480.1866497

K. Madduri, "Hybrid Parallel Programming for Massive Graph Analysis," Proc. SIAM Annual Meeting 2010 (July 2010). url http://www.graphanalysis.org/workshop2010.html

J. Orlin, K. Madduri, K. Subramani, M. Williamson; "A Faster Algorithm for the Single Source Shortest Path Problem with Few Distinct Positive Lengths," J. of Discrete Algorithms 8(2), 189-198, Elsevier (June 2010). url: http://dx.doi.org/10.1016/j.jda.2009.03.001

K. Subramani, C. Tauras, K. Madduri; "Space-time tradeoffs in negative cycle detection An empirical analysis of the Stressing Algorithm," Appl. Math. and Comp. 215(10), 3563-3575, Elsevier (January 2010). url: http://dx.doi.org/10.1016/j.amc.2009.10.053

V. Agarwal, K. Madduri, D.A. Bader, F. Petrini; "Combinatorial Algorithm Design on the Cell Processor," Scientific Computing with Multicore and Accelerators, J. Kurzak et al. (editors), CRC Press (in press). url: http://www.taylorandfrancis.com/books/details/9781439825365/

D.A. Bader, C. Heitsch, K. Madduri; "Large-scale Network Analysis," Graph Algorithms in the Language of Linear Algebra, J. Kepner and J. Gilbert (editors), SIAM (in press). url: http://www.siam.org/books/series/se.php

K. Madduri, "SNAP," Encyclopedia of Parallel Computing, D.A. Padua (editor-in-chief), Elsevier, in press. url: http://www.springer.com/computer/swe/book/978-0-387-09765-7

K. Madduri,"Betweenness Centrality," Encyclopedia of Parallel Computing, D.A. Padua (editor-in-chief), Elsevier (in press). url: http://www.springer.com/computer/swe/book/978-0-387-09765-7

D.A. Bader, K. Madduri; "Computational Challenges in Emerging Combinatorial Scientific Computing Applications," Combinatorial Scientific Computing, U. Naumann and O. Schenk (editors), CRC Press (in press). url: http://www.crcpress.com/product/isbn/9781439827352

CS-Yang-C LB10029 Computational Techniques for Non-crystalline X-ray Diffraction Imaging

C. Yang, Z. Wang, S. Marchesini; "Orientation Determination for 3D Single Molecule Diffraction Imaging," Proceedings of SPIE Conference, San Diego, Image Reconstruction from Incomplete Data VI, vol. 7800, pages 78000P-1 - 78000P-10 (August, 2010).

F. R. N. C. Maia, C. Yang, S. Marchesini; "Compressive auto-indexing in femtosecond nanocrystallography," to appear in Ultramicroscopy, 2010; also available at: arXiv:1011.3072 [physics optics], Nov 12, 2010. 
F. R. N. C. Maia, A. MacDowell, S. Marchesini, H. A. Padmore, D. Parkinson, C. Yang; "Compressive Phase Contrast Tomography," Proceedings of SPIE Conference, San Diego, Image Reconstruction from Incomplete Data VI, vol. 7800, pages 78000F-178000F-5 (August, 2010); also available at: arXiv:1009.1380 [physics.optics] Sept 7, 2010.

ESD-Brodie LB09035 Uncovering the Mechanistic Basis for Soil Microbial Community Response to Altered Precipitation Patterns.

K. Chourey, J. Jansson, N. Verberkmoes, M. Shah, K.L. Chavarria, L.M. Tom, E.L. Brodie, R.L. Hettich; "Direct Cellular Lysis/Protein Extraction Protocol For Soil Metaproteomics," J. of Proteome Research 9(12), 6615-22. Epub 2010 Nov 15. PubMed PMID: 20954746 (Dec $3^{\text {rd }}$ 2010). url: http://pubs.acs.org/doi/abs/10.1021/pr100787q

ESD-Commer LB10033 Enhanced Subsurface Fluid Characterization Using Joint Hydrological and Geophysical Imaging

M. Commer, G.A. Newman, K.H. Williams, S.S. Hubbard; "Three-dimensional spectral induced polarization data inversion for complex resistivity," accepted for publication in Geophysics.

M. Commer, S.A. Finsterle, M.B. Kowalsky, G.A. Newman; "Enhanced Subsurface Fluid Characterization Using Joint Hydrological and Geophysical Imaging," AGU Fall Meeting 2010, 13-17 December 2010, San Francisco, California.

M.B. Kowalsky, S.A. Finsterle, M. Commer, K.H. Williams, A. Englert, D. Sassen, D.Newcommer, S.S. Hubbard; "On parameterization of the inverse problem for estimating aquifer properties with tracer data and additional characterization data," draft for submission to $J$. of Hydrology.

ESD-Jannson-C LB08033 Carbon Uptake and Partitioning in Plants and Algae

C. Jansson, S. Wullschleger, U. Kalluri, G.T. Tuskan; "Phytosequestration: Carbon biosequestration by plants and the prospects for genetic engineering," BioScience $\mathbf{6 0}$, 685-696 (2010). url: http://www.bioone.org/doi/pdf/10.1525/bio.2010.60.9.6

E. Mangelsen, J. Kilian, K. Harter, C. Jansson, D. Wanke, E. Sundberg; "Transcriptome analysis of high-temperature stress in developing barley caryopses: Early stress responses and effects on storage compound biosynthesis," Mol. Plant. in press (available online: doi: $10.1093 / \mathrm{mp} / \mathrm{ssq} 058)$. url: http://mplant.oxfordjournals.org/content/early/2010/10/05/mp.ssq058.full.pdf

E. Mangelsen, D. Wanke, J. Kilian, E. Sundberg, K. Harter, C. Jansson; "Significance of light, sugar, and amino acid supply for diurnal gene regulation in developing barley caryopses," Plant Physiol. 153, 14-33 (2010). url: http://www.plantphysiol.org/cgi/reprint/153/1/14

D. Beyenne, Y. Baguma, S.B. Mukasa, C. Sun, C. Jansson; "Characterization and role of isoamylase1 (Meisa1) gene in cassava," African Crop. Sci. J. 18, 1-8. (2010). url: http://ajol.info/index.php/acsj/article/viewFile/54187/42713 
ESD-Jansson-J LB08034 Microbiomics of Complex Microbial Communities in Environmental $\underline{\text { Samples }}$

\section{Publications}

R. Mackelprang, M.P. Waldrop, K.M. DeAngelis, K.L. Chavarria, S.J. Blazewicz, E.M. Rubin, J.K. Jansson: "Deep metagenome sequencing illuminates permafrost response to thaw," submitted to Nature.

C. Jernberg, S. Lofmark, C. Edlund, J.K. Jansson; "Long-term impacts of antibiotic exposure on the human intestinal microbiota," Microbiology (in press).

B. Willing, J. Dicksved, J. Halfvarson, A. Andersson, M. Lucio, Z. Zeng, G.Jarnerot, C. Tysk, J.K. Jansson, L. Engstrand; "A pyrosequencing study in twins shows that GI microbial profiles vary with inflammatory bowel disease phenotypes," Gastroenterology 139,1844-1854.

H. Jakobsson, C. Jernberg, A.F. Andersson, M.Sjöland-Karlsson, J.K. Jansson, L. Engstrand; "Short-term antibiotic treatment has differing long-term impacts on the human throat and gut microbiome," PLoS One 5(3), 1-12(e9836).

A. Khoruts, J. Dicksved, J.K. Jansson, M. J. Sadowsky; "Changes in the composition of the human fecal microbiome following bacteriotherapy for recurrent Clostridium difficileassociated diarrhea," J. of Clinical Gastroenterology 44:354-360 (2010).

E. Wessen, K. Nyberg, J.K. Jansson, S. Hallin; "Responses of bacterial and archaeal ammonia oxidizers to soil organic and fertilizer amendments under long-term management," Applied Soil Ecology 45:193-200 (2010).

K. Hjort, M. Bergström, M. Adesina, J.K. Jansson, K. Smalla, S. Sjöling; "Chitinase genes revealed and compared in bacterial isolates, DNA extracts and a metagenomic library from a phytopathogen suppressive soil," FEMS Microbiology Ecology 71:197-207 (2010).

J. Dicksved, J. Halfvarson, M. Rosenquist, G. Järnerot, C. Tysk, J. Apajalahti, L. Engstrand, J.K. Jansson; "Molecular analysis of the gut microbiota of identical twins with Crohn's disease," The ISME Journal 2:716-727 (2008).

L. Jäderlund, V. Arthurson, U. Granhall, J.K. Jansson; "Specific interactions between arbuscular mycorrhizal fungi and plant growth/promoting bacteria as revealed by different combinations," FEMS Microbiology Letters 287:174-180 (2008).

A. Edlund, F. Hårdeman, J.K. Jansson, S. Sjöling; "Active bacterial community structure along vertical redox gradients in Baltic Sea sediment," Environmental Microbiology 10, 2051-2063 (2008).

A. Edlund, J.K. Jansson; "Use of bromodeoxyuridine immunocapture to identify psychrotolerant phenanthrene-degrading bacteria in phananthrene-enriched polluted Baltic Sea sediments," FEMS Microbiology Ecology 65:513-525 (2008).

\section{Books and Book Chapters}

B. Willing, J.K. Jansson; "The Gut Microbiota: Ecology and Function," in M. Sadowsky and R.L. Whitman (eds), The Fecal Bacterial, ASM Press, Washington, DC. ISBM number: 978-1-55581-608-7 (in press).

W.-T. Liu and J.K. Jansson (eds), Environmental Molecular Microbiology, Caister Academic Press, UK (2010, 231 pp).

J. Prosser, J.K. Jansson, W.-T. Liu; "Nucleic-acid-based characterization of community structure and function," in W.-T. Liu and J.K. Jansson (eds), Environmental Molecular Microbiology, Caister Academic Press, pp. 63-86 (2010). 
J. Dicksved, L. Zhao, J.K. Jansson; "Human Environment," in W.-T. Liu and J.K. Jansson (eds), Environmental Molecular Microbiology, Caister Academic Press, pp 167-190 (2010).

ESD-Liu LB09013 Impact of Climate Change on Soil Water Dynamics in Arid Areas

H.H. Liu, "Impact of Climate Change on Groundwater Recharge in Dry Areas: An Ecohydrology Approach," submitted to J. of Hydrology.

ESD-Oldenburg LB10017 CO2 as Cushion Gas for Compressed Air Energy Storage in Subsurface Reservoirs

C. M. Oldenburg, L. Pan; "Use of Carbon Dioxide as a Cushion Gas for CAES," 2nd Compressed Air Energy Storage (CAES) Conference \& Workshop, Columbia University, New York, NY, October 20-21, 2010.

C.M. Oldenburg, L. Pan; "Coupling Geologic Carbon Sequestration and Compressed Air Energy Storage,” ICEPAG 2011, Costa Mesa, California, February 8-10, 2011.

ESD-Salve LB09023 Identifying and Predicting Climate Change Impacts on the Land-Based Components of the Water Cycle

R. Salve, "Backfill Impacts on Moisture Measurements in Fractured Rock," submitted to Ground Water Monitoring \& Remediation.

R. Salve, "A Sensor Array System for Profiling Moisture in Unsaturated Rock and Soil," submitted to Hydrological Processes.

R. Salve, S. Finsterle, W.E. Dietrich; "Flow Through Fractured Rock in a Steep Hillslope," in preparation for submission to Water Resources Research.

R. Salve, E. Gasperikova, D.M. Rempe, J. Peterson; "Application of Electrical Resistivity Tomography for Hillslope Hydrology Studies," in preparation for submission to Water Resources Research.

D.M. Rempe, R. Salve, E. Gasperikova, J. Oshun, W.E. Dietrich, I. Fung; "Hydrogeomorphic Processes in Hillslopes, Rivers, and Landscapes," Geological Society of America Annual Meeting, Denver, CO, October 2010.

R. Salve, W.E. Dietrich, D.M. Rempe, J. Oshun, I. Fung; "Recharge along a Steep Hillslope," Fall Meeting, American Geological Union, San Francisco, CA, December 2010.

J. Oshun, R. Salve, D.M. Rempe, W.E. Dietrich, I. Fung; "Fractured Bedrock Storm Flow: A New Pathway for Runoff Generation," Fall Meeting, American Geological Union, San Francisco, CA, December 2010.

E. Gasperikova, R. Salve, D.M. Rempe, J. Peterson; "Investigating Hillslope Hydrology with Electrical Resistivity Tomography," Symposium on the Application of Geophysics to Environmental and Engineering Problems, Charleston, SC, April 2011.

ESD-Torn et al LB10022 Biological Carbon Sequestration: Fundamental Research on Biological Carbon Capture and Soil Carbon Stabilization

C. Jansson, T. Northen; "Calcifying cyanobacteria - the potential of biomineralization for carbon capture and storage," Current Opinion in Biotechnology 21, 365-371 (2010).

E. Brodie, C. Castanha, C. Santee, C. Chavaria, A. Crane-Droesch, M. Schmidt, J. Jansson, M. Torn; "Molecular analysis of soil microbial response to biochar," in preparation to be submitted to ISME Journal. 
K. Chourey, J. Jansson, N. VerBerkmoes, M. Shah, K. Chavarria, L. Tom, E. Brodie, R. Hettich; "A direct cellular lysis/protein extraction protocol for soil metaproteomics," J. of Proteome Research (in press).

EG-Battaglia LB08028 Advanced Silicon Detectors for Future Short Pulse X-ray Sources

M. Battaglia, P. Denes et al; "Radiation hardness studies on CMOS monolithic pixel sensors," Nucl. Instrum. Meth. A 624, 425 (2010).

S. Mattiazzo, M. Battaglia, P. Denes, et al; "Total Dose Effects on a FD-SOI Technology for Monolithic Pixel Sensors," IEEE Trans. Nucl. Sci. 57(4), 2135 (2010).

P. Giubilato, M. Battaglia, P. Denes, et al; "Tests of monolithic pixel sensors in Silicon On Insulator technology," to appear in Nucl. Instrum. Meth. A (2011).

Y. Arai, M. Battagla, P. Denes, et al; "Developments of SOI monolithic pixel detectors," Nucl. Instrum. Meth. A 623, 186 (2010).

D.B. Thorn, M. Battaglia, et al; "Spectroscopy of betatron radiation emitted from laserproduced wakefield accelerated electrons," Rev. Sci. Instrum. 81, 10E325 (2010).

G. Plateau, M. Battaglia, et al; "X-ray emission from electron betatron motion in a laserplasma accelerator," in Proc. of the 2010 Advanced Accelerator Concepts Workshop, Annapolis, MD, June 2010.

EG-Prestemon LB10018 High-Temperature Superconductors for Compact X-ray FEL's

S. Prestemon et al., "High Performance Short-Period Undulators Using High Temperature Superconductor Tapes," Proceedings PAC 2009, Vancouver, Canada.

S. Prestemon and R. Schlueter, "Undulator options for soft X-ray free electron lasers," Proceedings FEL 2009, Liverpool, UK.

S. Prestemon et al., "Development and Analysis of HTS-Undulator Components for FEL Applications," accepted for publication IEEE Transactions on Applied Superconductivity.

EETD-Bazjanac LB09003 Embedded Engineering, Construction Materials, and HVAC Components in Modular Energy Systems Simulation

V. Bazjanac, "Space boundary requirements for modeling of building geometry for energy and other performance simulation," CIB W78, Proc. 27th conf., Applications of IT in the AEC Industry, Cairo, November 2010.

T. Maile, V. Bazjanac, M. Fischer; "Simulating building energy performance for comparison with measured performance," submitted for publication in J. of Building Performance Simulation.

V. Bazjanac, T. Maile, J.T. O’Donnell, C.M. Rose, N. Mrazović, S. Monna; Space boundaries for building energy performance simulation, LBNL Report number pending (December 2010).

V. Bazjanac, T. Maile, J.T. O’Donnell, C.M. Rose, N. Mrazović, "Use of data in the AEC industry - questionable practices with serious implications," in preparation to be submitted to Science.

EETD-Coughlin, Fridley LB10004 Physically-Based Accounting for Resource Use in New Energy Pathways

K. Coughlin, "A Mathematical Analysis of Full Fuel Cycle Energy Use," preprint to be submitted to Energy. 
K. Coughlin, D. Fridley, A. Sturges; "Estimating the production potential for truly carbonneutral biomass-based energy," in preparation for possible submission to Energy.

EETD-Gadgil LB10041 Developing a Fuel-Efficient Cook Stove for Haiti

A. Gadgil, K. Booker; "Cookstove Dissemination in Haiti: Improving Collaboration and Information-Sharing," in press Boiling Point 59 (September 2010). url: http://www.hedon.info/boilingpoint.

K. Booker, J. Granderson, T. Han. N. Yang; "Report on Results of WBT for Charcoal Stoves for Haiti," draft LBL Technical Report.

EETD-Kerr LB09012 Development of Novel Improved Capacitors for Pulse Power Applications

J Chmiola, M.M. Doeff, J.B. Kerr; "Strategies for improving electrochemical capacitor performance," Abstract \#235, 218th ECS Meeting, Las Vegas NV, October 12, 2010.

J.Chmiola and J. Kerr on each of two manuscripts in preparation, expect to submit to $J$. of the Electrochemical Society.

EETD-Maddalena LB09015 Relating Tissue Residues to Indoor Chemical Sources in a Bayesian Framework Synthesis of Chemistry, Pharmacokinetics, and Biomarkers

A.B. Lobscheid, R. Maddalena, A.B. Barrett, T.E. Mckone; "Advancing Exposure Assessment Methods for Semi-Volatile Organic Chemicals using Indoor Modeling, Monitoring Data, and Biomarker Measurements," in preparation for submission to $J$. of Exposure Science and Environ. Epidemiology.

R. Goth-Goldstein, A.B. Lobscheid, B. Noth; "PAHs in the Indoor Environment: A Review on the Linkages between Residential Exposure Concentrations, Human Biomarker Data, and Health Effects," in preparation for submission to J. of Exposure Science and Environ. Epidemiology.

EETD-Price, Black LB09020 Self-Tuning Building Energy Model

One publication anticipated.

EETD-Wetter LB10028 Building Systems for Net Zero Energy Buildings

M. Wetter, W. Zuo, T.S. Nouidui; "Recent Developments of the Modelica Buildings Library for Building Energy and Control Systems," draft submitted to 2010 Modelica Conference.

Y. Yang, A. Pinto, A. Sangiovanni-Vincentelli, Q. Zhu; “A Design Flow for Building Automation and Control Systems," to be published in the 31st Real-Time Systems Symposium, 2010.

GN-Biggin LB09004 Measurement of Protein/ DNA Binding in vitro and in vivo Using Single Molecule Approaches

One publication anticipated

GN-Pennacchio LB09019 Assessing Epigenomic Approaches for Gene Enhancer Discovery

A. Visel, M.J. Blow, Z. Li, et al; "ChIP-seq Accurately Predicts Tissue-Specific Activity of Enhancers," Nature 457, 854-8 (Feb. 12, 2009).

A. Visel, E.M. Rubin, L.A. Pennacchio; "Genomic Views of Distant-Acting Enhancers," Nature 461, 199-205. (Review, Sep. 10, 2009). 
M.J. Blow, Z. Li, D.J. McCulley, et al; “ChIP-Seq Identification of Weakly Conserved Heart Enhancers," Nature Genetics 42(9), 806-10 (Sep. 2010).

L.A. Pennacchio, A. Visel; "Limits of Sequence and Functional Conservation," Nature Genetics 42(7), 557-8 (News and Views, Jul. 2010).

LSD-Blakely LB10040 Synchrotron-Based Microtomography for Functional Analysis of Normal Tissue and Tumor Molecular Markers, and Their Perturbation by Low-Dose Radiation Exposure

E. Blakely, K. Bjornstad, C. Rosen, et al; "High-resolution tomographic imaging of tumors and organs in mice (poster)," Life Sciences Division Retreat Abstract Book (September $23,2010)$.

A. Thompson, K. Bjornstad, C. Rosen, et al; "High-resolution tomographic imaging of tumors and organs in mice (poster)," ALS Users' Meeting Abstract Book (October 14, 2010).

D. Parkinson, "Challenges in image processing in tomographic data sets (oral presentation)," LCLS/SSRL Users' Meeting and Workshops (October 17-21, 2010).

E. Blakely, K. Bjornstad, C. Rosen, et al; "High-resolution tomographic imaging of murine tumors and organs," in preparation for submission to Nature Methods.

LSD-Comolli LB09006 Linking Genomics, Proteomics and Ultrastructural Characterization of Microbial Communities and Their Viruses

B. Knierim, B. Luef, P. Wilmes, M. Auer, R.I. Webb, L.R. Comolli, J.F. Banfield; "Concomitant high-resolution phylogenetic identification and ultrastructural characterization of mixed microbial consortia," submitted to Nature Methods.

L.R. Comolli, D. Baum, R. Harris, et al; "A portable cryo-plunger device for the preparation of cry-electron microscopy intact environmental samples directly in the field," submitted to Microscopy and Microanalysis.

LSD-Mao LB10013 Identification of Genetic Networks Controlling Susceptibility to RadiationInduced Carcinogenesis

Z. Li, Q. Zhang, J.H. Mao, et al; "An HDAC1-binding domain within FATS bridges p21 turnover to radiation-induced tumorigenesis," Oncogene 29, 2659-71 (2010).

Z. Hu, G. Huang, A. Sadanandam, et al; "The expression level of HJURP has an independent prognostic impact and predicts the sensitivity to radiotherapy in breast cancer," Breast Cancer Research 12, R18 (2010).

M.S. Hung, Y.C. Lin, J.H. Mao, Z. Xu, C.T. Yang, D.M. Jablons L. You; "Functional polymorphism of the CK2 $\alpha$ intronless gene plays oncogenic roles in lung cancer," PLOS One 5:e11418 (2010).

J. Climent, J. Perez-Losada, D.A. Quigley, et al; "Deletion of the hPER3 gene on chromosome 1p36 in recurrent ER-positive breast cancer," J. of Clinical Oncology, 28, 3770-3778 (2010).

M.G. Chun, J.H. Mao, C.W. Chiu, A. Balmain, D. Hanahan; "Polymorphic genetic control of tumor invasion in a mouse model of pancreatic neuroendocrine carcinogenesis," PNAS 107, 17268-73 (2010).

J. Pérez-Losada, A. Castellanos-Martín, J.H. Mao; “Cancer Evolution and Individual Susceptibility," Integrative Biology (in press).

R. Xu, J.H. Mao; "Gene transcriptional networks integrate microenvironmental signals in human breast cancer," Integrative Biology (in press). 
LSD-Tainer LB09025 Bio Energy Technologies and Science Integrated Efficiently (BETSIE)

A. Cvetkovic, AL. Menon, M.P. Thorgersen, et al; "Microbial metalloproteomes are largely uncharacterized," Nature 466(7307), 779-82 (Aug. 5, 2010).

R.P. Rambo, J.A. Tainer; "Bridging the solution divide: comprehensive structural analyses of dynamic RNA, DNA, and protein assemblies by small-angle X-ray scattering," Curr. Opin. Struct. Biol. 20(1), 128-37 (Feb. 2010).

S. Classen, I. Rodic, J. Holton, G.L. Hura, M. Hammel, J.A. Tainer; "Software for the highthroughput collection of SAXS data using an enhanced Blu-Ice/DCS control system," $J$. Synchron. Rad. 17(6), 774-81 (Nov. 2010).

LSD-Wang-D LB10035 Multinozzle Arrays for Single Cell Metabolomics

D. Wang, S. Bodovitz; "Single Cell Analysis: the New Frontier in 'Omics'," Trends in Biotechnology 28(6), 281-290 (Cover review, June 2010). PMCID: PMC2876223.

LSD-Wiese LB10039 Functional Characterization of NUCKS- a Potential Cancer

Susceptibility Locus Required for Recombination

C. Wiese, A. Kuo, R. Rahnama, E. Dray, A.C. Østvold, P. Sung, D. Schild; "NUCKS, a RAD51AP1 paralog, protects human cells from the cytotoxic effects of X-rays," Abstract and Poster Presentatio,. 56 ${ }^{\text {th }}$ Annual Meeting of the Radiation Research Society, Maui, September 25-29, 2010.

C. Wiese, E. Dray, T. Groesser, S.B. Seidler, A. Kuo, A.C. Østvold, P. Sung, D. Schild; "NUCKS, a RAD51AP1 paralog, is required for DNA repair by homologous recombination, in preparation for submission to PNAS.

MSD-Clarke, Haeffner LB10003 Heating Rates of Planar Ion Traps for Quantum Information

N. Daniilidis, S. Narayanan, S.A. Moeller, et al; "Fabrication and heating rate study of microscopic surface electrode ion traps," accepted for publication in New Journal of Physics. arXiv:1009.2834, url: http://arxiv.org/abs/1009.2834)

MSD-Milliron LB09017 Mixed Ionic and Electronic Transport in Solution-Processed Inorganic Nano-Composites (TEM)

R. Tangirala, J.L. Baker, A.P. Alivisatos, D.J. Milliron; "Modular inorganic nanocomposites by conversion of nanocrystal superlattices," Angew. Chem. Int. Ed. 49, 2878 (2010).

R.Y. Wang, R. Tangirala, G. Garcia, D.J. Milliron; "Understanding mixed ionic and electronic conduction in inorganic nanocomposites," draft.

MSD-Minor LB09018 In situ Electromechanical Probing in a Transmission Electron Microscope (TEM)

J.X. Zhang, B. Xiang, Q. He, et al; "Large-field-induced strains in a lead-free piezoelectric material," Nature Nanotechnology (in press).

MSD-Schoenlein LB09034 X-ray Studies of Charge-Order Dynamics in Complex Materials

S.Y. Zhou, Y. Zhu, M.C. Langner, Y.-D. Chuang, P. Yu, W.L. Yang, A.G. Cruz Gonzalez, M. Rini, N. Tahir, Y.-H. Chu, R. Ramesh, D.-H. Lee, Y. Tomioka, Y. Tokura, Z. Hussain, R.W. Schoenlein; draft for submission to Phys. Rev. Lett. 
MSD-Smit, Xu LB09029 Tuning the Self-Assembly of Membrane Proteins

J.M Rodgers, M. Webb, B. Smit; "Alcohol solubility in a lipid bilayer: Efficient grandcanonical simulation of an interfacially active molecule," J. Chem. Phys. 132 (2010). doi:06410710.1063/1.3314289

F.J.M de Meyer, A. Benjamin, J. M. Rodgers, Y. Misteli, B. Smit; "Molecular Simulation of the DMPC-Cholesterol Phase Diagram,” J. Phys. Chem. B 114, 10451-10461 (2010). doi:10.1021/jp103903s

F.J.M de Meyer, J.M. Rodgers, T.F. Willems, B. Smit; "Molecular Simulation of the Effect of Cholesterol on Lipid-Mediated Protein-Protein Interactions," Biophys. J. 99, 36293638 (2010). doi:10.1016/j.bpj.2010.09.030

MSD-Tirrell LB10021 Structured Charged Polymers

C. Schneider, A. Jusufi, R. Farina, P. Pincus, M. Tirrell, M. Ballauff; "Stability behavior of anionic spherical polyelectrolyte brushes in the presence of La(III) counterions," Phys. Rev. E. 82, 011401 (2010).

MSD-Urban LB08044 Understanding How Nanoscale Interfaces Modify Predicted Optical, Vibrational, and Electronic Properties

A. Schwartzberg, T. Kuykendall, S. Aloni, J. Urban; "Nanoscale White-Light Generation Driven by Defect-Mediated 2-Photon Absorption in Gallium Nitride Nanowires," to be submitted to Phys. Rev. B.

A. Weber-Bargioni, A. Schwartzberg, J. Urban, P. Schuck, S. Cabrini; "Reproducible Fabrication of Optical Antennae on Scanning Probe Tips for High Resolution Spectral Raman Mapping of Carbon Nano Tubes," draft.

A. Schwartzberg, T. Kuykendall, S. Aloni, J. Urban; "Determination of Sub-DiffractionLimit Gallium Nitride Nanowire Crystallographic Orientation by Diffraction-Limited Raman Imaging," draft.

A. Schwartzberg, T. Kuykendall, S. Aloni, J. Urban; "Internally Produced White-Light for Fabry-Pérot interferometry of Single Gallium Nitride Nanowires," draft.

A. Schwartzberg, T. Kuykendall, S. Aloni, J. Urban; "Gallium Nitride Nano-wires as a Directional White Light Source with Sub Band-gap Excitation and Observed Changes in Refractive Index by Fabry-Perot Spectroscopy," submitted oral presentation to Material Research Society, November 2009 meeting.

A. Schwartzberg, T. Kuykendall, S. Aloni, J. Urban; "Directional white-light generation on the nanoscale," submitted oral presentation to American Chemical Society, March 2010 meeting.

A. Schwartzberg, J. Urban; “Crystallographic Orientational Imaging of Gallium Nitride Nanowires via Confocal Raman Imaging," submitted oral presentation to Material Research Society, Winter 2010 meeting.

A. Weber-Bargioni, A. Schwarzberg, M. Cornaglia, J. Urban, D. Ogletree, S. Cabrini, P. Schuck; "Reproducible Fabrication of Optical Antennae on Scanning Probe Tips for High Resolution Spectral Raman Mapping of Carbon Nano Tubes," submitted oral presentation to Material Research Society, Winter 2010 meeting.

MSD-Wang-F LB10025 Integrated Photonic, Electronic and Spintronic Devices Based on Graphene

J. Horng, C.-F. Chen, B. Geng, et al; "Crude Conductivity of Dirac Fermions in Graphene," submitted to Nano Lett. 
MSD-Yang-P LB10030 Design and Surface Properties of Semiconductor Nanowires

H. Ko, K. Takei, R. Kapadia, et al; "Ultrathin compound semiconductor on insulator layers for high performance nanoscale transistors," Nature 468, 286-289 (2010).

H. Lee, J. Wu, J.C. Grossman; "Enhancing thermoelectric power factor with highly mismatched isoelectronic doping," Phys. Rev. Lett. 104, 016602 (2010).

J. Cao, Y. Gu, W. Fan, et al; "Extended Mapping and Exploration of the Vanadium Dioxide Stress Temperature Phase Diagram," Nano Lett. 10, 2667 (2010).

G.F. Brown, J.W. Ager III, W. Walukiewicz, J. Wu; "Finite Element Simulations of Compositionally Graded InGaN Solar Cells," Solar Energy Mater. \& Solar Cells 94, 478 (2010).

MSD-Zhang-Y LB10036 Surface-Selective Synthesis of Graphene Nanoribbons on Nanowire Templates

A. Ismach, C. Druzgalski, S. Penwell, et al; "Direct chemical vapor deposition of graphene on dielectric surfaces," Nano Letters 10, 1542-1548 (April 2, 2010). url: http://pubs.acs.org/doi/abs/10.1021/n19037714

NSD-Klein LB08011 A 100 km3 Neutrino Detector for Ultra High Energy Neutrinos

L. Gerhardt, S. Klein; "Electron and photon interactions in the regime of strong LPM suppression," Phys. Rev. D82, 074017 (2010).

L. Gerhardt, S. Klein, T. Stezelberger, S. Barwick, K. Dookayka, J. Hanson, R. Nichol; “A prototype station for ARIANNA: a detector for cosmic neutrinos," Nucl. Instrum. \& Meth. A624, 85 (2010).

S. Buitink for the ARIANNA Collaboration, "ARIANNA: A $100 \mathrm{~km}^{\wedge} 3$ scale detector for extremely high-energy neutrinos," poster presented at the $22^{\text {nd }}$ European Cosmic Ray Symposium, August 3-6, 2010, Turku, Finland.

S. Klein, "Radiodetection of Neutrinos," invited talk presented at Neutrino 2010, June 14-19, 2010, Athens, Greece. The writeup of this talk will appear in the proceedings and is available at http://arxiv.org/PS cache/arxiv/pdf/1012/1012.1407v1.pdf.

NSD-Vetter-K LB09026 Ultra-Sensitive Ge Detectors for Low-Background Physics

Experiments

P.N. Luke, M. Amman, J. Detwiler, J. Loach, R. Martin, A. Poon, J. Qian, C. S. Tindall, K. Vetter; "Low-Noise Low Mass Front-End Electronics for Low-Background Physics Experiments using Germanium Detectors," in preparation for submission to Nucl. Instr. and Methods.

NSD-Yuan-F LB09031 Theoretical Study of Nucleon Structure

B. Xiao, F. Yuan; "Non-Universality of Transverse Momentum Dependent Parton Distributions at Small-x, Phys. Rev. Lett. 105, 062001 (2010).

D. Boer, Z. Kang, W. Vogelsang, F. Yuan; "Test of the Universality of Naive-time-reversalodd Fragmentation Functions," Phys. Rev. Lett. 105, 202001 (2010).

B. Xiao, F. Yuan; "Initial and Final State Interaction Effects in Small-x Quark Distributions," Phys. Rev. D82, 114009 (2010).

Z. Kang, F. Yuan, J. Zhou; "Twist-three Fragmentation Function Contribution to the Single Spin Asymmetry in pp Collisions," Phys. Lett. B691, 243 (2010). 
S. Brodsky, B. Pasquini, B. Xiao, F. Yuan; "Phases of Augmented Hadronic Light-Front Wave Functions," Phys. Lett. B687, 327 (2010).

B. Pasquini, F. Yuan, Sivers; "Boer-Mulders functions in Light-Cone Quark Models," Phys. Rev. D81, 114013 (2010).

H. Avakian, A.V. Efremov, P. Schweitzer, F. Yuan; "The transverse momentum dependent distribution functions in the bag model," Phys. Rev. D81, 074035 (2010).

Z. Kang, F. Yuan; "Dihadron Azimuthal Correlation from Collins Effect in Unpolarized Hadron Collisions," Phys. Rev. D81, 054007 (2010).

J. Zhou, F. Yuan, Z. Liang; "Transverse momentum dependent quark distributions and polarized Drell-Yan processes," Phys. Rev. D81, 054008 (2010).

F. Dominguez, B. Xiao, F. Yuan; "kt-factorization for Hard Processes in Nuclei," submitted to Phys. Rev. Lett. e-Print: arXiv:1009.2141 [hep-ph].

PBD-Fletcher LB09008 Engineering Environmental Sensitivity in an Artificial Cell

D. Richmond, E. Schmid, S. Martens, J. Stachowiak, N. Liska, D. Fletcher; "Forming giant vesicles with controlled membrane composition, asymmetry, and contents," submitted to PNAS.

R. Rounsevell, L. Nilsson, D. Fletcher; "Symmetry Breaking and Global Remodeling in Spherical Actomyosin Networks," draft.

PBD-Groves LB09010 Applications of Hybrid Live Cell Synthetic Devices for Cancer Research

K. Salaita, P.M. Nair, R.S. Petit, R.M. Neve, D. Das, J.W. Gray, J.T. Groves; "Physical Manipulation of EphA2 Spatial Organization Alters Cellular Response to Ephrin-A1," Science 327, 1380-1385 (12 March 2010). url: http://www.sciencemag.org/cgi/content/full/327/5971/1380

P.M. Nair, K. Salaita, R.S. Petit, J.T. Groves; "Using Nanopatterned Lipid Membranes to Investigate the Role of Receptor Organization in Intercellular Signaling," Nature Protocols (in press).

PBD-Liphardt et al LB08013 Light-Boosted Fermentation in the Yeast Saccharomyces Cervisiae

J.M. Walter, D. Greenfield, J. Liphardt; "Potential of light-harvesting proton pumps for bioenergy applications," Curr. Opin. Biotechnol. 21, 265-270 (June, 2010).

J.M. Walter, J. Liphardt; "Light-harvesting proton pumps for bioenergy applications," whitepaper prepared for Jonathan Burbaum, Program director, ARPA-E Applied Biotechnology for Transportation Fuels.

PBD-Scheller LB10019 Engineering of Drought and Heat Tolerance in Bioenergy Crops

U. Christensen, A. Zygadlo-Nielsen, J. Harholt, P. Ulvskov, P. Canlas, P. Ronald, H.V. Scheller, "Characterization and Biological Function of Mannosylglycerate Synthase in $S$. moellendorfii," draft.

J. Harholt, I. Sørensen, A. Roberts, W. Willats, H.V. Scheller, B.L. Petersen, J.A. Banks, P. Ulvskov: "A Comparative Study of Cell Wall Structure and Glycosyltransferase Repertoire of the Spike Moss Selaginella moellendorffii," draft.

H.V. Scheller; "Biomass - Design of plants for biofuel production and other novel applications," Workshop on strategic plant research - solutions to global societal challenges, Copenhagen, Denmark, March 24, 2010. 
U. Christensen; "Engineering stress tolerance in crops by accumulation of mannosylglycerate," Poster at Gordon Conference "Salt and Water Stress in Plants", Switzerland, June 2010.

U. Christensen; "Engineering stress tolerance in higher plants by accumulation of mannosylglycerate," presentation at University of Copenhagen, Faculty of Life Sciences, June 2010.

PBD-Yildiz LB09033 Control of Intraflagellar Transport in Chlamydomonas Cells

S.M. Shih, F. Kocabas, W. Marshall, A. Yildiz; "Single Molecule Analysis of Intraflagellar Transport in Chlamydomonas Cells," in preparation to be submitted to Cell.

PH-Nomura LB10016 Theoretical Studies of Dark Matter Beyond the Standard Model

G. Ellor, H.-S. Goh, L.J. Hall, Y. Nomura; "Environmentally Selected WIMP Dark Matter with High-Scale Supersymmetry Breaking," Phys. Rev. D81, 095003 (2010).

C. Cheung, Y. Nomura, J. Thaler; “Goldstini,” JHEP 03, 073 (2010).

C. Cheung, J. Mardon, Y. Nomura, J. Thaler; "A Definitive Signal of Multiple Sypersymmetry Breaking,” JHEP 07, 035 (2010).

PH-Smoot, Leauthaud LB08020 Decoding Dark Energy with Weak Gravitational Lensing

A. Leauthaud, et al; "A Weak Lensing Study of X-ray Groups in the COSMOS survey: Form and Evolution of the Mass-Luminosity Relation," ApJ 709 (1), 97-114 (2010). url: http://adsabs.harvard.edu/abs/2010ApJ...709...97L

J. Rhodes, et al; "The Effects of Charge Transfer Inefficiency (CTI) on Galaxy Shape Measurements, Pub. Astron. Soc. Pac. 122 (890), 439-450 (2010). url: http://adsabs.harvard.edu/abs/2010PASP..122..439R

PH-White, Padmanabhan LB08046 Calibrating Baryon Acoustic Oscillations for Future Dark Energy Experiments

$\mathrm{X}$. Xu, M. White, N. Padmanabhan, et al; "A new statistic for analyzing baryon acoustic oscillations," ApJ 718, 1224 (2010).

M. White, A. Pope, J. Carlson, K. Heitmann, S. Habib, P. Fasel, D. Daniel, Z. Lukic; "Particle mesh simulations of the Lyman-alpha forest and the signature of baryon acoustic oscillations in the intergalactic medium," ApJ 713, 383 (2009).

H.-J. Seo, J. Eckel, D. J. Eisenstein, et al; "High-precision predictions for the acoustic scale in the non-linear regime," ApJ 720, 1650 (2009).

Y. Noh, M. White, N. Padmanabhan; "Reconstructing baryon oscillations," Phys. Rev. D 80, 123501 (2009).

Cross-Div.-Auer et al LB08047 Integrated Tools in Multiscale Imaging

A.R. Lowe, J.J. Siegel, P. Kalab, M. Siu, K. Weis, J.T. Liphardt; "Selectivity mechanism of the nuclear pore complex characterized by single cargo tracking," Nature 467, 600-603 (2010). PMID 20811366

B. Knierim, R.I. Webb, L.M, M. Desai, B. van Leer, T. Goddard, P. Hugenholtz, K.L. McDonald, M. Auer; "Multiscale Three-Dimensional Organization of the Termite Hindgut Elucidated by FIB/SEM," in preparation for submission to J. Structural Biology. 
Cross-Div.-Milliron et al LB10015 Multifunctional Window Coatings for High-Performance Buildings

R.J. Mendelsberg, S.H.N. Lim, D.J. Milliron, A. Anders; "High Rate Deposition of High Quality ZnO:Al by Filtered Cathodic Arc," presented at the Fall Meeting of the Material Research Society (MRS), November 30, 2010; manuscript under review.

Cross-Div.-Schroeder et al LB08018 Coupled Process Models, Separations, and Monitoring for Advanced Nuclear Fuel Cycles

R.K. Crockett, P. Colella, D.T. Graves; "A Cartesian Grid Embedded Boundary Method for Solving the Poisson and Heat Equations with Discontinuous Coefficients in Three Dimensions," submitted to J. of Comp. Physics.

R.K. Crockett, P. Colella; "Conjugate Heat Transfer Simulations of Free and Forced Convection from Conducting Cylinders with Volumetric Sources Using Cartesian CutCell Methods," in preparation for submission to the International Journal of Heat and Mass Transfer.

J.E. Bickel, M. Laufer, L. Li, A.C. Cisneros, P.F. Peterson; “Conceptual Design, Experiments, and Analysis for the Core of an FHR-16 Test Reactor," International Congress on Advances in Nuclear Power Plants (ICAPP'10, Paper 10130), San Diego, CA. June 13-17, 2010.

M.R. Laufer, "Granular Dynamics in Pebble Bed Reactor Cores,” Doctoral Dissertation, University of California, Berkeley, CA; anticipated May 2011.

R. Copping, S.J. Teat, B. Jeon, M. Janousch, T. Tyliszczak, A. Canning, N. GrønbechJensen, D.K. Shuh; "Towards Equatorial Planarity About Uranyl, Structure, and Bonding in N-donor Uranyl(VI) Complexes," in preparation to be submitted to Inorganic Chemistry.

P.A. Glans, G. Szigethy, D. Demoin, T. Tyliszczak, J. Xu, J. Guo, K.N. Raymond, D.K. Shuh; "Actinide Science with a Soft X-ray Scanning Transmission X-ray Microscope (STXM)," Mater. Res. Soc. Symp. Proc. 1264, BB06-05 (2010).

B. Jeon, N. Grønbech-Jensen, R. Copping, A. Canning, D.K. Shuh; "Coordination Characteristics of Uranyl BBP Complexes: Electronic Structure Analysis," draft.

B. Jeon, G. Szigethy, N. Grønbech-Jensen, R. Copping, A. Canning, K.N. Raymond, D.K. Shuh; "Electronic Structure Calculations of Ce/Pu-maltol Ligand Complexes," draft. 
